\title{
Self and other regarding motives in decision making under uncertainty
}

Citation for published version (APA):

Cettolin, E. (2012). Self and other regarding motives in decision making under uncertainty. [Doctoral Thesis, Maastricht University]. Datawyse / Universitaire Pers Maastricht. https://doi.org/10.26481/dis.20120530ec

Document status and date:

Published: 01/01/2012

DOI:

10.26481/dis.20120530ec

Document Version:

Publisher's PDF, also known as Version of record

\section{Please check the document version of this publication:}

- A submitted manuscript is the version of the article upon submission and before peer-review. There can be important differences between the submitted version and the official published version of record.

People interested in the research are advised to contact the author for the final version of the publication, or visit the DOI to the publisher's website.

- The final author version and the galley proof are versions of the publication after peer review.

- The final published version features the final layout of the paper including the volume, issue and page numbers.

Link to publication

\footnotetext{
General rights rights.

- You may freely distribute the URL identifying the publication in the public portal. please follow below link for the End User Agreement:

www.umlib.nl/taverne-license

Take down policy

If you believe that this document breaches copyright please contact us at:

repository@maastrichtuniversity.nl

providing details and we will investigate your claim.
}

Copyright and moral rights for the publications made accessible in the public portal are retained by the authors and/or other copyright owners and it is a condition of accessing publications that users recognise and abide by the legal requirements associated with these

- Users may download and print one copy of any publication from the public portal for the purpose of private study or research.

- You may not further distribute the material or use it for any profit-making activity or commercial gain

If the publication is distributed under the terms of Article $25 \mathrm{fa}$ of the Dutch Copyright Act, indicated by the "Taverne" license above, 
Self and other regarding motives in decision making under uncertainty 
Self and other regarding motives in decision making under uncertainty.

(C) Copyright Elena Cettolin, Maastricht 2012

All rights reserved. No part of this publication may be reproduced, stored in a retrieval system, or transmitted, in any form, or by any means, electronic, mechanical, photocopying, recording or otherwise, without the prior permission in writing from the author.

This book was typeset by the author using $\mathrm{IAT}_{\mathrm{E}} \mathrm{X}$.

ISBN 9789461951531

Printed in the Netherlands by Datawyse 


\title{
Self and other regarding motives in decision making under uncertainty
}

\author{
Dissertation \\ to obtain the degree of Doctor at \\ Maastricht University, \\ on the authority of the Rector Magnificus, \\ Prof. dr. G.P.M.F. Mols, \\ in accordance with the decision of the board of Deans, \\ to be defended in public \\ on Wednesday 30 May 2012, at 12:00 hours \\ by

\section{Elena Cettolin}

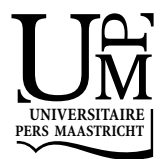




\section{Supervisor:}

Prof. dr. Arno Riedl

\section{Assessment Committee:}

Prof. dr. Thomas Dohmen (Chairman)

Dr. Martin Strobel

Prof. dr. Bertil Tungodden (Norwegian School of Economics) 


\section{Acknowledgments}

Almost 5 years have passed since the day I arrived to the Netherlands, in September 2007. Having always been an accomplished student, I expected that doing research would not be much more difficult than writing a good exam. I could not have been more mistaken as research is an active, creative but disciplined effort. Learning from others' books and papers is only a tiny part of the story. Luckily, I could always count on my supervisor: Arno patiently taught me how to structure my thoughts and shape them into meaningful research questions. He was never tired to provide feedback on my immature drafts, discuss the design of our experiments, encourage me when I was feeling frustrated. Arno, I am very grateful for your enduring support and I am looking forward to continue working on our projects. I would also like to acknowledge the members of the degree committee, Thomas Dohmen, Martin Strobel and Bertil Tungodden, for assessing my thesis and providing me with useful feedback. The presence of colleagues and friends at Maastricht University has been immensely important through this journey. I would like to thank Martin, Ronald, Sasha, Andres, Matt and Friederike for their valuable comments on my projects. Thank you Franziska: working together has been fun and productive, I can only hope that we continue along this way. Thank you Nicole and Elke, your kindness and helpfulness is very much appreciated. Guillaume and Andy, my hosts when I visited NYU, and the whole department at CESS made my experience there simply great by creating every day a friendly and productive atmosphere. Thank you all! 
My dearest friends Anna, Charlie and Myrthe: I want to thank you because you always gave me the feeling I could count on you. The ROA gang (Jan, Eric, Martin, Olivier) together with Maciek, the Tiger, Diogo, Jojo and Julio (we miss you!) has been the best "sifting valve" one can possibly imagine. Many thanks to all of you. Even though we were geographically very far most of the time, Marta, Giulia, La Magnana, Betty, Lu, Serena and Giovanni have always been present in my life: thank you all, your friendship is very precious to me.

Finally I would like to Antonio and my parents for their unconditional love: questo libro e' dedicato a voi, dalla prima all'ultima pagina.

The research undertaken in this thesis partially benefited from a financial support by The Netherlands Organization for Scientific Research (NWO) under the grant Open Competition-400-09-451.

Elena Cettolin

Maastricht, May 2012 


\section{Contents}

1 Introduction $\quad 1$

2 Revealed Incomplete Preferences under Uncertainty 5

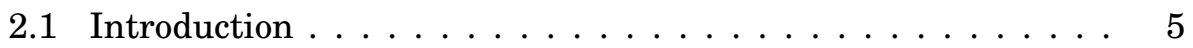

2.2 An experiment on choice under uncertainty . . . . . . . 8

2.3 Results . . . . . . . . . . . . . . . . . 14

2.3.1 Are preferences of indecisive subjects incomplete? . . . . 22

2.4 An experiment to test the completeness

of preferences . . . . . . . . . . . . . . . . 24

2.4 .1 Results . . . . . . . . . . . . . . . . 27

2.5 Individual characteristics . . . . . . . . . . . . . . . 29

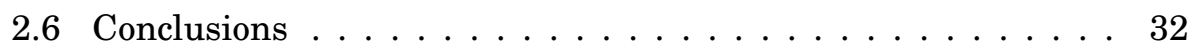

2.7 Appendix . . . . . . . . . . . . . . 33

2.7.1 $\alpha$-maxmin Expected Utility Theory . . . . . . . . . . 33

2.7.2 Cumulative Prospect Theory . . . . . . . . . . . . . . . 35

2.7.3 Regret Aversion . . . . . . . . . . . . . . 36

2.7.4 Instructions of the experiment . . . . . . . . 43

3 Fairness and Uncertainty $\quad 51$

3.1 Introduction . . . . . . . . . . . . . . . . 51

3.2 Distributive justice when outcomes are uncertain . . . . . . 54

3.2.1 The general problem . . . . . . . . . . . . 54

3.2 .2 Theoretical discussion $\ldots \ldots \ldots \ldots \ldots$ 
3.3 The Benevolent Dictator experiment . . . . . . . . . . . . . 60

3.4 Results . . . . . . . . . . . . . . . . . . . 66

3.4 .1 Fairness ideals . . . . . . . . . . . . . . . . . . . 74

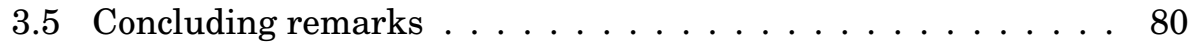

3.6 Appendix . . . . . . . . . . . . . . . . . 82

3.6.1 Fairness ideals: analytical derivation . . . . . . . . 82

3.6.2 Answers in the debriefing questionnaire . . . . . . 86

3.6.3 Instructions of the experiment $\ldots \ldots \ldots$. . . . 87

4 Generosity in the Face of Uncertainty 101

4.1 Introduction . . . . . . . . . . . . . . . . . . 101

4.2 The experiment . . . . . . . . . . . . . . . . . 104

4.3 Results . . . . . . . . . . . . . . . . . . 110

4.4 Fairness norms . . . . . . . . . . . . . . . . . . 115

4.4.1 Decision making model and estimation . . . . . . 117

4.4.2 Estimates of the decision making model . . . . . . . . 118

4.5 Conclusions . . . . . . . . . . . . . . . . . . . 122

4.6 Appendix . . . . . . . . . . . . . . . . . . . . . 124

4.6 .1 Tables . . . . . . . . . . . . . . . . . . 124

4.6.2 Instructions of the experiment $\ldots \ldots \ldots \ldots$

5 Risk Taking and Risk Sharing - Does Accountability Matter? 139

5.1 Introduction . . . . . . . . . . . . . . . . . . . . . 139

5.2 Experimental design . . . . . . . . . . . . . . . . . . . 143

5.3 Theoretical predictions and hypotheses . . . . . . . . 146

5.4 Results . . . . . . . . . . . . . . . . . . . . . . . 149

5.4.1 Risk preferences and risk exposure . . . . . . . . . 150

5.4 .2 Risk sharing . . . . . . . . . . . . . . . . 151

5.5 Discussion and conclusions . . . . . . . . . . . . . . . 157

5.6 Appendix . . . . . . . . . . . . . . . . . 158

5.6.1 Instructions of the experiment $\ldots \ldots \ldots \ldots 158$

5.6 .2 Number of observations . . . . . . . . . . . . 163

$\begin{array}{ll}\text { Bibliography } & 165\end{array}$

$\begin{array}{lc}\text { Curriculum Vitae } & 173\end{array}$ 


\section{Chapter 1}

\section{Introduction}

As the future is mostly unknown, we primarily make decisions under uncertainty. We choose how to invest our money, which career to pursue and how to educate our children without perfect knowledge of the ensuing consequences. Even the results of trivial decisions, like taking or not taking an umbrella when leaving home, are affected by which one of the many possible states of nature materializes. Decision theory describes this kind of problems as a choice between uncertain prospects. Once all the constitutive elements of the prospects are considered, we can select the prospect that gives us the higher expected utility, that is the largest benefit in expectation. Even if we may seldom find ourselves thinking about utilities and probabilities, we may agree that decision theory provides a quite satisfactory representation of how decisions should be, and in fact are, made.

Introspection however reveals that making a choice is sometimes difficult: we may be indecisive, for example, because we do not know which choice object we prefer or because the probability of future events is hard to quantify. In my first chapter I experimentally investigate (with Arno Riedl) individuals' choice behavior in situations where uncertainty may lead to indecisiveness. Our novel design allows observing a so far unexplored behavioral regularity: approximately half of the decision makers in our experiment avoid several choices between risky and ambiguous prospects by delegating them to a random chance device. We consider several decision 
making theories and test whether they are able to account for the observed choice behavior. Our data do not only question the predictive power of traditional decision making models but also appear inconsistent with approaches that explicitly account for behavioral aspects of decision making, such as probability weighting and regret aversion. We suggest that the observed choice anomaly is the consequence of individuals holding incomplete preferences over ambiguous and risky prospects. More precisely, a decision maker that has multiple priors over an ambiguous event may entertain as many representations of the corresponding ambiguous prospect. The existence of multiple representations may in turn impede the comparison of prospects. Importantly, our results suggest that indecisiveness stirs people towards options that allow avoiding active choices. Further, they present a caveat to the presence of ambiguity averse preferences: rather than being averse to ambiguity per-se, half of the subjects in our experiment dislike making choices under uncertainty.

Choosing whether to take or not an umbrella from home is an act that does not require depriving others of the possibility to find shelter from the rain. However, economics usually deals with scarcity, that is the problem of humans having needs and wants in a world where resources are limited. Defining what constitutes a fair allocation of a scarce resource seems particularly problematic in the presence of uncertainty. For instance, how should the likelihood of survival, if at all, be taken into account when ranking patients for organ transplant? What is the appropriate compensation for high risk jobs as compared to riskless ones? These examples are only a few of the many where allocations need to be defined in the presence of uncertainty. In my second chapter I study (together with Arno Riedl) individuals' normative fairness judgments in distribution problems where resources have to be allocated before, or without, knowing the true state of nature that influences their final distribution.

We find that, compared to a situation where uncertainty is absent, people hold very heterogeneous ideas about fair allocations, which also depend on their own preferences for uncertainty. In theory, four fairness ideals based on general distributive justice principles may be endorsed: our data show that each ideal motivates a significant share of the observed allocations. Fur- 
thermore, the interpretation of distributive principles is context dependent. Indeed, we observe that it is systematically related to the degree of uncertainty characterizing the distribution problem.

Experiments in economics and in psychology have largely demonstrated that a considerable fraction of individuals care about others' outcomes in a positive way. More recently, authors started investigating the conditions under which individuals' generosity diminishes. Giving to others appears to be particularly under jeopardy in situations where the link between individuals' generosity and observable final outcomes is blurred. In my third chapter I investigate (together with Arno Riedl) how generosity is affected by uncertainty. Participants to the experiment are asked to distribute a given amount between themselves and an anonymous recipient, their own final position (or the position of the recipient) being uncertain at the moment of the division. By exploiting the results of our second paper, this study also allows testing whether uncertainty induces individuals to adopt self-serving biased definitions of fairness in order to act more selfishly.

We find that individuals' generous behavior is not significantly affected by the presence of uncertainty. However, when accounting for the possible fairness norms that are relevant in the studied distribution problems, we observe that some individuals adopt a biased fairness view in order to retain more money. This however happens only when uncertainty affects the outcome of the decision maker. Not only fairness views seem to be unbiased when the recipient is instead affected by uncertainty: we also observe that the more risk averse an individual the more generously he acts towards the recipient facing uncertainty.

Uncertainty is not only characterizing active decision making: humans are often directly affected by risky events that can be at best influenced by their decisions. In order to decrease their vulnerability to events that would negatively affect their financial situation, individuals can create risk sharing arrangements. The size of the insurance industry and of national social security systems evidently demonstrates the economic relevance of risk sharing arrangements. In my fourth chapter I experimentally investigate (with Franziska Tausch) the determinants of individuals' willingness to engage in collective risk sharing. We hypothesize that the more risk exposure is per- 
ceived as a deliberate choice the smaller the support for risk sharing. The role plaid by individuals' risk preferences and their own and others' exposure to risks is also investigated. We find that, irrespective of their own risk attitude, individuals are less willing to share risks when the other member in the pair deliberately exposed himself to a high risk. This result is stronger the more extreme the potentially bad consequences of risk taking. We also observe that individuals who are only occasionally taking a high risk tend to share more risk with individuals who also chose a high exposure. 


\section{Chapter 2}

\section{Revealed Incomplete Preferences under Uncertainty}

\subsection{Introduction}

Most decision making models assume that individuals are always able to pair-wise compare two, or more, available options. In other words, it is assumed that decision makers have complete preferences. However, completeness has long been recognized as a problematic assumption. The following sentence by Aumann (1962) describes this issue concisely: 'Of all the axioms of utility theory, the completeness axiom is perhaps the most questionable. Like others of the axioms, it is inaccurate as a description of real life; but unlike them, we find it hard to accept even from the normative viewpoint'. Starting with Aumann (1962) decision theorists have proposed models that allow a decision maker to be occasionally indecisive. More recently, authors have linked incomplete preferences with multi-objective (or multi-self) decision making under risk and certainty (see, for instance, Ok, 2002 and Dubra, 2004). In these models preferences may be incomplete when the decision maker (from now onwards DM) is characterized by two selfs that pursue possibly orthogonal objectives. As an example, when choosing a meal an agent's 'healthy' self may be confronted with her 'hedonic' self. 
Existing models are also capable of representing situations where preferences between uncertain prospects are incomplete because of Knightian uncertainty (Bewley 2002 and Gilboa et al., 2010). Incompleteness is ascribed to the presence of multiple representations of the same prospect, which originate from the DM set of priors on the uncertain event. In other words, incomplete preferences stem from the fact that the DM lacks information to determine which option is best. Last but not least, the relaxation of the completeness axiom has been essential for the development of theoretical models that can account for behavioral anomalies like the status quo bias (Masatlioglu and Ok, 2005), the endowment effect (Mandler, 2004) and preference reversal (Eliaz and Ok, 2006).

Albeit important, so far theoretical accomplishments were not paired with rigorous empirical evidence on the existence of incomplete preferences. Experiments in economics and psychology document that complex choice situations may lead decision makers to be indecisive but the observed indecisiveness may be attributed to factors other than incompleteness of preferences. For instance, Shafir and Tversky (1992) show that large choice sets constituted by similar items induce people to stick to the status quo, even though each of the available items is preferred to it. This result suggests that a potentially complete preference relation becomes incomplete in some circumstances: however, competing explanations exists, like decision costs that increase in the number of alternatives or regret aversion. ${ }^{1}$

The major obstacle to obtaining empirical evidence on incompleteness resides in one of the founding blocks of modern economics, the revealed preferences approach. Choices reveal preferences and as a consequence the incompleteness of a preference relation is difficult to judge from observed choice behavior. Further, within this approach indecisiveness cannot be distinguished from indifference, the latter meaning that two options are considered equally attractive while the former indicating the inability to pair-wise

\footnotetext{
${ }^{1}$ Similar experiments were also run by Redelmaier and Shafir (1995) who find that the tendency to stick to the status quo is common among professionals. See also Shafir (1993) and Simonson (1989) for experiments describing behavior that could be the product of incomplete preference relations.
} 
rank options. ${ }^{2}$

In this paper we experimentally investigate choice behavior in decision situations where preferences may be incomplete because of uncertainty. The study is composed of two main experiments. In the first experiment subjects are asked to make a series of choices between a risky two-outcomes prospect (known probabilities) and an ambiguous two-outcomes prospect (unknown probabilities). Outcomes are the same in all prospects and the level and source of ambiguity is kept constant in all decision situations. Instead, the winning probability of the risky prospect varies in each decision situation, ranging from certainty to win the high outcome to certainty to loose it. In each decision situation, we allow subjects to avoid choosing between prospects. This can be done by selecting a third option which consists of a chance device that assigns either the ambiguous or the risk prospect to the DM, with equal probability.

We find that half of the subjects in our experiment avoid several choices between prospects, this evidence representing a so far unexplored behavioral anomaly. Indeed, we show that the observed behavior cannot be reconciled with the most popular models of decision making under uncertainty, such as Maxmin Expected Utility (Gilboa and Schmeidler, 1989), Cumulative Prospect Theory (Tversky and Kahneman, 1992) and Regret theory (Loomes and Sugden, 1982).

In the second experiment we test whether subjects' behavior may be rationalized by an incomplete preference relation due to uncertainty, as in Gilboa et al. (2010). To this end we increase the high outcome associated to the ambiguous prospect, keeping everything else equal to the first experiment. If the observed behavioral anomaly is the product of incomplete preferences, we should observe that subjects still avoid making choices but in different decision situations. We find that subjects' choices in the second experiment are largely consistent with the hypothesis that avoid active choices when preferences are incomplete.

Our results confirm the importance of relaxing completeness for the formal

\footnotetext{
${ }^{2}$ An exception is constituted by Eliaz and Ok, 2006. The authors relax the Weak Axiom of Revealed Preferences to propose a theory of decision making that allows identifying indecisiveness and indifference from observable choice behavior.
} 
modeling of decision making under uncertainty. In particular, the observed behavioral anomaly calls attention on the kind of decision rules that individuals may adopt in situations where preferences are potentially incomplete. The observed choice behavior also implies that subjects are not averse to ambiguity when they do not have to choose between uncertain prospects. Ambiguity aversion (Ellsberg, 1961) is considered an important violation of rationality and decades of research in experimental economics show that the majority of subjects display such aversion (for a review see Camerer and Weber, 1992). We show that the possibility to avoid active decision making is sufficient for ambiguity aversion to disappear. Lastly, we find a strong gender effect as female subjects are much more likely to avoid active choice.

The reminder of the paper is organized as follows. Section 2.2 describes the design of the first experiment and section 2.3 presents and discusses the results. Section 2.4 presents our second experiment and its results. Section 2.5 describes which individual characteristics are related to indecisiveness. Conclusions are drawn in section 2.6.

\subsection{An experiment on choice under uncertainty}

The main purpose of this experiment is to investigate people's choice behavior in decision situations where uncertainty may cause preferences to be incomplete. To this end, in the first part of the experiment subjects face a series of incentivized choices between risky and ambiguous prospects, with the option to remain indecisive between them. Being indecisive in a certain choice situation implies that a fair chance device selects which prospect, risky or ambiguous, is assigned to the subject in that situation.

It has been shown that accounting for the way people think about probabilities can highly improve the descriptive validity of models of decision making under risk (Tversky and Kahneman, 1979). In the first part of our experiment participants have to make choices between prospects with known probabilities (risk) and prospects with unknown probability (ambiguity). Hence, we are interested in exploring whether subjects' perception of probabilities contributes to explaining their choices. The second part of 
the experiment consists of a series of incentivized choices between lotteries and sure amounts which allow us to jointly estimate the parameter values of subjects' utility and probability weighting function. Finally, we are interested in exploring how subjects' cognitive abilities and thinking styles affect decision making under uncertainty. The last part of the experiment includes psychological questionnaires that measure such characteristics.

Note that subjects were not informed about the structure of the experiment: instructions were administered on the computer screen before the beginning of each part. In the following the experimental procedures for the three parts are described in detail.

Choices under uncertainty In the first part of the experiment subjects go through a series of choices between one ambiguous and one risky prospect. Before that, subjects select a color (red or black) which will be their winning one in each choice situation. Two urns are used to generate uncertainty. The risky urn, Urn A, contains 100 balls colored red and black, and its composition varies in each choice situation. The proportion of colored balls is modified in steps of 5, starting with 100 red balls and ending with 100 black ones. Hence, there are 21 choices in total, all displayed on one table. ${ }^{3}$ Figure 2.1 displays a screen shot of the table. Risky prospects offer increasingly worse (better) chances to win for subjects betting on red (black). The ambiguous urn, Urn B, also contains 100 red and black balls but their proportion is unknown to the subjects as well as to the experimenter. Both urns are visibly placed in the experimental lab and subjects are informed that they are free to inspect their content after the experiment is over. A colleague of us composed the ambiguous urn, being free to put as many red and as many black balls in the urn, provided that the total number had to be 100. The urn was then sealed and nobody except the composer, who was in no way involved in the experiment, was aware of its composition. In each of the 21 choice situations subjects choose whether they want to bet on their winning color from the urn with known composition or from the ambiguous one. Subjects can also avoid to actively choose one of the prospects by selecting the middle

\footnotetext{
${ }^{3}$ In the empirical literature, tables with paired lotteries are commonly used to elicit risk attitudes (Holt and Laury, 2002) and less often to elicit attitude to uncertainty (Cohen et al., 1987).
} 
option in the decision table. In formulating this option in the experiment we use the expression "I am indifferent between the two urns". The word indifferent seems a quite natural choice in this context as in everyday language it is used to indicate lack of a precise preference. Further other terms, such as indecisive or insecure, may be more likely to create experimental demand effects. We did not impose any consistency requirement on subjects' choices, such as to allow switching from one type of urn to the other only once.

Incentive compatibility is obtained with the random-lottery method. ${ }^{4}$ Before making their choices, participants are informed that each of the 21 choice situations is equally likely to be selected for payment. If a winning ball is drawn from the chosen urn the subject earns $€ 15$, otherwise she/he earns nothing. If a choice situation where the subject was indecisive is relevant for payment, a fair chance device is used to select one of the two urns. All the described procedure took place publicly at the end of the experiment so that subjects could witness how the chance devices were operated.

After making the 21 choices, subjects proceed to a different screen where they are asked to provide their best estimate of the ambiguous urn's composition. Subjects can click on any of 20 check boxes that indicate the number of red balls in the ambiguous urn in intervals of 5 balls. Note that we explicitly mention the possibility to click on more than one check box. This task is not incentivized.

Probability weighting and utility function The second part of the experiment is designed to jointly estimate the utility and probability weighting function parameters at the individual level (Fehr-Duda et al., 2006). Subjects' certainty equivalents are elicited for a series of 33 two outcomes lotteries. Table 2.2 shows the outcomes (in Euro) and probabilities employed. For each lottery subjects are presented with a decision screen that contains a description of the lottery and a list of 20 equally spaced sure amounts, ranging from the lottery's highest to lowest outcome. In order to facilitate comprehension, the lottery odds are expressed both in percentage points and with the aid of a pie chart. Figure 2.2 displays a decision screen.

In each row of the decision screen subjects have to make a choice between

\footnotetext{
${ }^{4}$ See $h t t p: / /$ people.few.eur.nl/wakker/miscella / debates/randomlinc.htm for a discussion on the appropriateness of the random-lottery incentives scheme.
} 
Figure 2.1: Screen shot of decision table in part 1 .

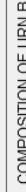

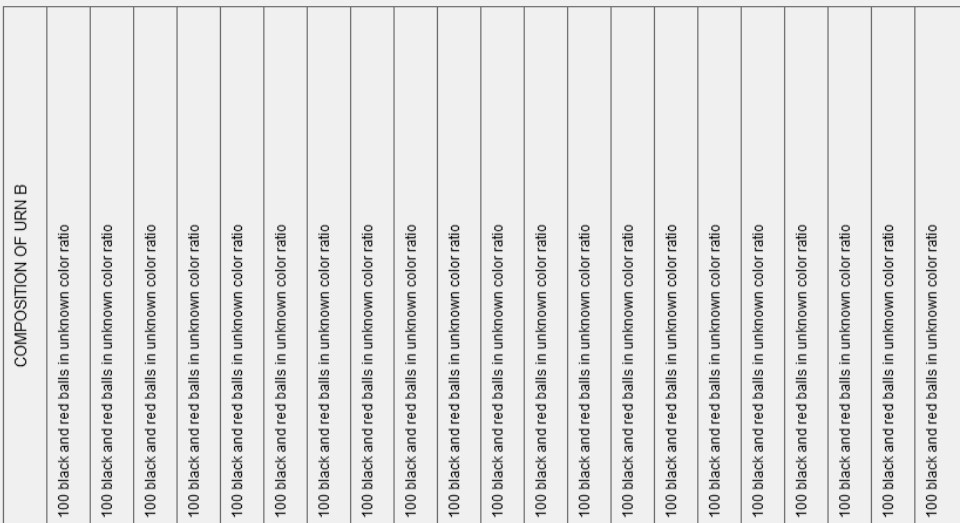

赵

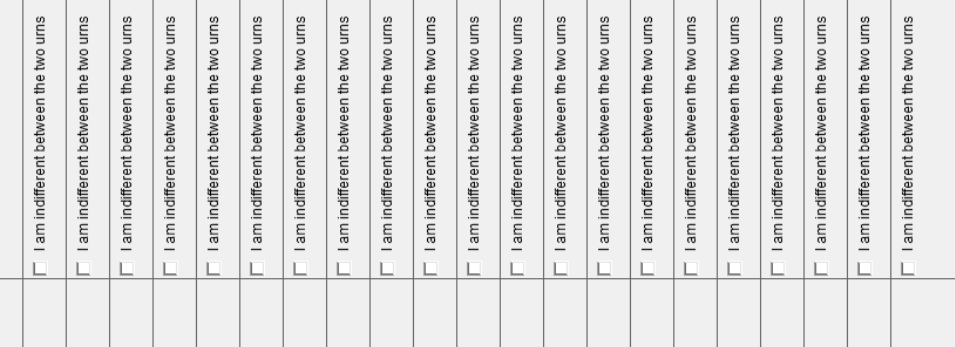

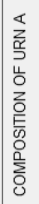


Figure 2.2: Screen shot of a typical decision screen of part 2.

\begin{tabular}{|c|c|c|c|}
\hline Decison șruation 1. & $\begin{array}{l}\text { oproun } \\
\text { Loluter }\end{array}$ & rourctrocese & 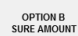 \\
\hline choice 1 & & $A<C B$ & 10. \\
\hline choice 2 & & $A r r B$ & 9.50 \\
\hline choice 3 & & $A r r B$ & 9. \\
\hline choice 4 & & Arra & 8.50 \\
\hline choice 5 & & $\operatorname{ArcB}$ & 8. \\
\hline choice 6 & & $\operatorname{Arca} \theta$ & 7.50 \\
\hline choice 7 & 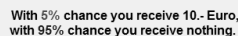 & $\operatorname{Arco}$ & 7.. \\
\hline choice 8 & & $\operatorname{AscB} \theta$ & 6.50 \\
\hline choice 9 & & 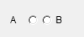 & 6. \\
\hline choice 10 & & 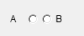 & 5.50 \\
\hline choce 11 & & $A r r B$ & 5. \\
\hline choive 12 & & $\operatorname{ArcB}$ & 4.50 \\
\hline choice 13 & & $A \operatorname{crs} B$ & 4. \\
\hline choice 14 & & $\operatorname{ArcB}$ & 3.50 \\
\hline choice 15 & & $\operatorname{ArcB}$ & 3. \\
\hline choice 16 & & $A \operatorname{crB} B$ & 2.50 \\
\hline choice 17 & & $\operatorname{ArcB}$ & 2. \\
\hline choice 18 & & $\operatorname{Acc} \theta$ & 1.50 \\
\hline choice 19 & & $A c r a$ & 1. \\
\hline choice 20 & & 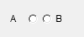 & 0.50 \\
\hline
\end{tabular}

the lottery and the sure amount. Certainty equivalents are then calculated as the arithmetic mean of the smallest sure amount preferred to the lottery and the consecutive sure amount on the list.

At the end of the experiment one decision screen and one row within the decision screen are randomly selected for payment. The relevant lottery is then publicly played out and earnings are added to those of the first part.

Psychological measures In the last part of the experiment subjects are asked to answer questions from the Cognitive Reflection Test (CRT) by Frederick (2005). The CRT is a 3 items test that measures the ability to reflect on a problem, the first answer that comes to ones' mind being always wrong. Furthermore, performance in the CRT has been found to be positively and significantly correlated with standard measures of cognitive ability. Subjects are rewarded with $€ 0.50$ for each correct answer and have a limited time to provide their answers.

Thereafter, subjects go through the 31 items of the Rational-Experiential Inventory (REI, Epstein et al., 1996). REI includes 19 items on a 5 points 
Table 2.1: Lotteries, $p_{1}$ indicates the probability of winning $x_{1}$ Euro.

\begin{tabular}{lrrlrrlrr}
\hline \hline$p_{1}$ & $x_{1}$ & $x_{2}$ & $p_{1}$ & $x_{1}$ & $x_{2}$ & $p_{1}$ & $x_{1}$ & $x_{2}$ \\
\hline 0.05 & 10 & 0 & 0.35 & 25 & 10 & 0.65 & 20 & 5 \\
0.05 & 20 & 5 & 0.45 & 10 & 0 & 0.65 & 25 & 10 \\
0.05 & 25 & 10 & 0.45 & 20 & 5 & 0.75 & 10 & 0 \\
0.1 & 5 & 0 & 0.45 & 25 & 10 & 0.75 & 20 & 5 \\
0.1 & 10 & 5 & 0.5 & 5 & 0 & 0.75 & 25 & 10 \\
0.1 & 25 & 0 & 0.5 & 20 & 5 & 0.9 & 5 & 0 \\
0.25 & 10 & 0 & 0.5 & 25 & 10 & 0.9 & 10 & 5 \\
0.25 & 20 & 5 & 0.55 & 20 & 5 & 0.9 & 25 & 0 \\
0.25 & 25 & 10 & 0.55 & 25 & 10 & 0.95 & 10 & 0 \\
0.35 & 10 & 0 & 0.55 & 10 & 0 & 0.95 & 20 & 5 \\
0.35 & 20 & 5 & 0.65 & 10 & 0 & 0.95 & 25 & 10 \\
\hline
\end{tabular}

scale that measure analytical-rational processing and 12 items, also on a 5 points scale, that measure engagement and confidence in one's intuitive abilities. Analytical and intuitive modes represent two fundamental ways in which people process information. In fact, differences in thinking modes can explain a wide variety of behaviors and possibly also to the way in which people make decisions under uncertainty. In the final part, subjects are asked a few socioeconomic questions and then they are privately paid out in cash and dismissed.

55 students from Maastricht University participated in the computerized experiment which was conducted in March 2009 in the Behavioral and Experimental Lab (BEElab) at the Maastricht University School of Business and Economics, using the Z-Tree software (Fischbacher, 2007). 90\% of the subjects were enrolled in the Faculty of Economics and Business Administration and $58 \%$ of them were male. The average age was 23 years. The experiment lasted on average 90 minutes and the average earnings per subjects were $€ 32.95$. 


\subsection{Results}

For ease of understanding and for convenience, in what follows subjects' choices in the first part of the experiment are recoded and then analyzed as if red had been selected as winning color by everybody. ${ }^{5}$

We find that as long as it gives a winning probability of at least 0.5 , the risky urn is the most common choice. However, when the winning probability drops to 0.45 the ambiguous urn becomes the most popular option for all consecutive choice situations. In other words, by varying the winning probability of the risky prospect between 1 and 0 , we observe that the majority of people prefers the risky prospect to the ambiguous one only in 11 out of 21 choice situations. If in each choice situation we assign half of the indecisive choices to the risky option and the other half to the ambiguous one, in a given situation we can use a binomial test to verify whether choices in favor of one option are significantly larger than 50\%. It turns out that choices favoring the risky prospect are significantly larger than $50 \%$ at the $1 \%$ significance level in all choice situations characterized by a winning probability $p \geq 0.5$. On the other hand, choices favoring the ambiguous prospect are significantly larger than $50 \%$ at the $1 \%$ significance level in all choice situations characterized by a winning probability $p \leq 0.4$. When the winning probability of the risky prospect is equal to 0.45 the number of choices favoring the risky prospect is not significantly different than the number of choices favoring the ambiguous prospect $(\mathrm{p}=0.14)$. This evidence suggests that subjects in the experiment are only moderately averse to ambiguity. Several investigations have shown that ambiguity aversion is negatively related to the decision maker's familiarity with the source of uncertainty (Fox and Tversky, 1995, Fox and Weber, 2002). In this experiment ambiguity is artificially generated in the laboratory using an urn with unknown composition, which is a rather unfamiliar source of uncertainty. Hence, since subjects have never participated in similar experiments before, the moderate aversion to ambiguity we observe cannot be explained by a sense of familiarity with the decision con-

\footnotetext{
${ }^{5}$ We exclude from the analysis 1 subject who switched back and forth between the three options of the first task 11 times, thus displaying random behavior. We also exclude 1 subject who displayed a highly inconsistent behavior in several lotteries of the second part.
} 
text.

In fact, the most striking feature emerging from our data is that a large number of subjects do not actively avoid the ambiguous prospects. Subjects are often indecisive, particularly for choice situations where the winning probability of the risky prospect is between 0.5 and 0.35 . When the winning probability is $0.5,36 \%$ of the subjects choose the fair chance device. Similarly, $37 \%$ of the subjects choose the fair chance device when the winning probability is 0.45 and $36 \%$ when the winning probability is 0.4 and 0.35 . In all the other choice situations less than $23 \%$ of the subjects choose the device. These figures already suggest that some subjects chose the fair chance device more than once. The histogram in Figure 2.3 confirms this by showing the relative frequency of choices in favor of the chance device. ${ }^{6}$

Figure 2.3: Relative frequency of indecisive choices.

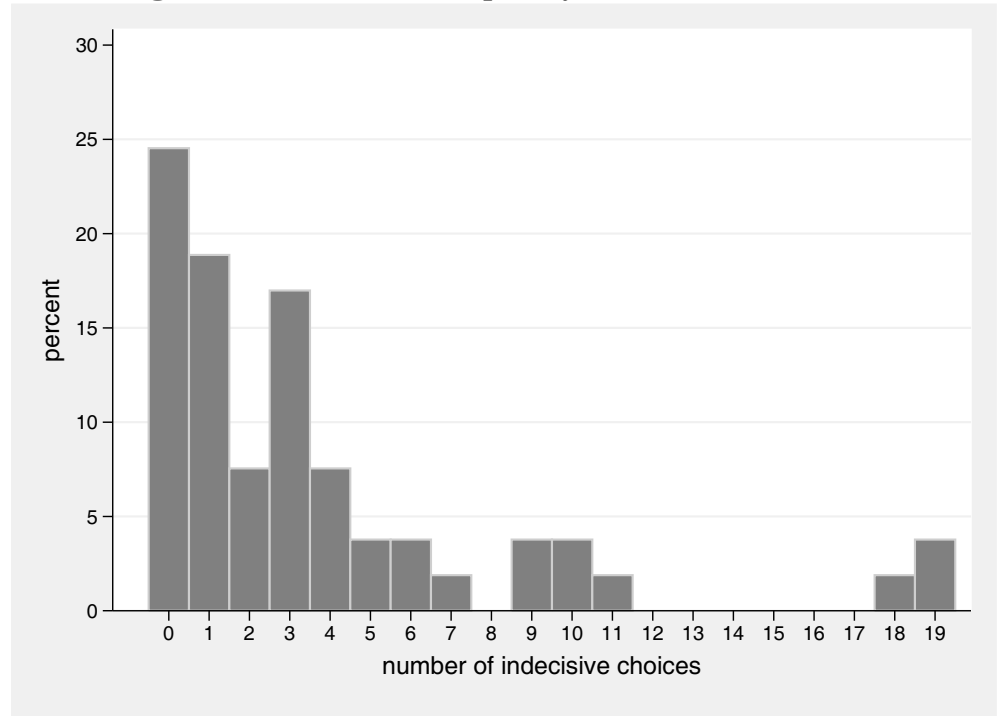

Subjective Expected Utility theory (SEU, Savage, 1954) is the equivalent of Expected Utility theory when probabilities are unknown to the decision

\footnotetext{
${ }^{6}$ The histogram does not include decisions made in the first and in the last choice situations, where the choosing the chance device may only be a mistake. In any case, the classification of subjects in indecisive and non-indecisive would not change if those choice situations are also included.
} 
maker. According to SEU theory subjects would choose the chance device only when the winning chance of the risky prospect equals their subjective prior on the ambiguous urn. Stated differently, subjects would choose the device when they are truly indifferent between the two prospects. The device is never chosen by only $24.5 \%$ of the subjects and $19 \%$ choose it exactly once. Hence, behavior $43.5 \%$ of the subjects is in accordance with SEU theory. The device was chosen exactly two times by $7.5 \%$ of the subjects. Even though this is not consistent with SEU theory, errors deriving from the relatively small likelihood difference that exists between two consecutive choices in the table may explain these choices.

The remaining $49 \%$ of the subjects choose the device in at least three consecutive choice situations. The most common situation is to select the fair chance device in three consecutive choices (17\% of the participants), followed by four choices (7.5\% of the participants). A few subjects even choose the fair chance device in almost all situations. Note that all subjects that choose the device exactly three times do this in consecutive situations of the table. Only $15 \%$ of the subjects do not display compact intervals.

Result 2.3.1. About half of the subjects are indecisive between risky and ambiguous prospects at least 3 times, mainly in consecutive choice situations.

In the following we refer to subjects that chose the chance device at most twice as "decisive" and to subjects who chose the chance device at least three times as "indecisive". Interestingly, self reported estimates on the composition of the ambiguous urn reveal that on average decisive subjects believe that there are 53 winning balls and indecisive subjects 48 winning balls, this difference being insignificant (Mann Whitney test $\mathrm{p}=0.16$ ). Notwithstanding this similarity in beliefs, SEU theory is clearly not suited to account for indecisive subjects' choices. ${ }^{7}$ In the following we show that many other theories of decision making under uncertainty are also unable to account for repeated choice of the chance device.

Multiple priors models In these family of models consider, for instance, $\alpha$ Maxmin Expected Utility ( $\alpha$-MEU) theory (Ghirardato, Maccheroni

\footnotetext{
${ }^{7}$ Actually, the limits of SEU theory in describing choice under uncertainty are known since Ellsberg's seminal two colors example (Ellsberg, 1961).
} 
and Marinacci, 2004), which is a development of Maxmin Expected Utility theory (Gilboa and Schmeidler, 1989) that explicitly accounts for attitude to ambiguity. In this model the decision maker may hold a set of priors on the ambiguous event and is characterized by an index $\alpha$ which captures attitude to ambiguity. Repeatedly choosing the chance device implies that either the prior on the ambiguous event or ones' attitude to ambiguity, the index $\alpha$, are revised in each choice situation. ${ }^{8}$ In our view, the assumption of continuous revision of beliefs and preferences does not appear as a plausible mechanism underlying choice behavior.

As a next candidate for explaining repeated choice of the chance device we consider a descriptive model of decision making under uncertainty, $\mathrm{Cu}$ mulative Prospect Theory (CPT, Tversky and Kahneman, 1992). CPT allows for the existence of sub-additive decision weights reflecting the idea that people have preferences over sources of uncertainty even when the beliefs associated with each source are the same (Tversky and Wakker, 1995). In general, more familiar and intelligible sources of uncertainty receive higher decision weights than less familiar ones. In our experiment, repeated choice of the chance device would imply that different probabilities values are all equally weighted. ${ }^{9}$ Thus, the more a subject is indecisive, the flatter the subject's probability weighting function. In other words, CPT may account for repeated choice of the chance device only if indecisive subjects are characterized by extreme insensitivity to likelihood changes. The existing empirical literature on probability weighting already suggests that this explanation can be ruled out because likelihood insensitivity, although widespread, is usually not too extreme (see, for instance, Abdellaoui, 2000). The second part of our experiment allows to directly test the descriptive validity of CPT by estimating the parameters of the value and probability weighting function at the individual level.

Probability weighting In order to make CPT operational, we have to assume specific functional forms for the value function $v(x)$ and for the probability weighting function $w(p)$. We use the specifications proposed by

\footnotetext{
${ }^{8}$ This can be easily proven, but it requires some tedious algebra. We invite the reader to refer to the appendix.

${ }^{9}$ Again, we invite the reader to refer to the appendix for a formal proof.
} 
Kahneman and Tversky (1992) because they combine goodness of fit with parsimony. Thus, we assume $v(x)=x^{\alpha}$, where $\alpha=1$ indicates a linear value function, $\alpha>1$ a convex one and $0<\alpha<1$ a concave value function and

$$
w(p)=\frac{p^{\gamma}}{\left(p^{\gamma}+(1-p)^{\gamma}\right)^{1 / \gamma}}
$$

where $\gamma=1$ indicates linear weighting. For values of $\gamma<1$ the function has an inverted-s shape and for $\gamma>1$ the function is s-shaped.

Under these parametric assumptions, we estimate the parameter values of $\alpha$ and $\gamma$ for each subject by minimizing the sums of squared distances (Wakker, 2009) that is:

$$
\sum_{i=1}^{33}\left(l_{i}-c e_{i}\right)^{2}
$$

where $l_{i}$ is the CPT value of lottery $i$ and $c e_{i}$ is the CPT certainty equivalent of lottery $i .{ }^{10}$ Subjects are free to switch back and forth between the lottery and the sure amount. Since the calculation of certainty equivalents requires subjects to switch from the lottery to the sure amount just once, we can only use decisions screens that meet this condition. However, since we need estimates of the relevant parameters for each individual we cannot exclude too many decisions. Thus, the following rule is employed: if a subject switches back and forth between the lottery and the sure amount in more than four lotteries, all her decisions are excluded from the experiment. In fact, only one subject had to be excluded from the analysis. Subjects with less than four inconsistent decision sheets are considered in the analysis but the inconsistent decision sheets are dropped.

Recall that the chance device is mostly chosen when the risky prospect has a winning probability that lies within 0.35 and 0.5 . Probabilities in the interval $[0.35,0.5]$ are all differently weighted if $\gamma \geq 0.37$. On the other hand, $\gamma \leq 0.25$ implies that all probabilities in the interval receive equal weight. Unless stated otherwise, all statistical tests in this paper are two tailed. We find that on average decisive and indecisive subjects are characterized by a nearly identical value of $\gamma$ (0.63 and 0.62 respectively) which is not signifi-

\footnotetext{
${ }^{10}$ To correct for heteroscedasticity prospects are normalized to uniform length.
} 
cantly different in the two groups (Mannn Whitney test $p=0.2$ ). ${ }^{11}$ Among decisive subjects, only 2 out of 27 are characterized by a $\gamma<0.37$ and nobody appears to hold a completely flat weighting function in the relevant probability interval. Similarly, only 2 out of 26 indecisive subjects are characterized by a $\gamma<0.37$ and nobody by a $\gamma \leq 0.25$. As a consequence, the hypothesis that indecisiveness follows from flatness of the probability weighting function can be ruled out.

Probabilistic choice We now consider the possibility that repeated choice of the chance device derives from a probabilistic choice process (Harrison, 2008). Probabilistic choice models extend traditional theories of decision making by allowing for decision errors. These models assume that the probability of choosing a prospect is not equal to one when its expected utility exceeds the expected utility of the alternative prospect. Instead, the likelihood of choosing a prospect is a function of the expected utility difference existing between the available prospects. The larger the expected utility difference, the higher the likelihood of choosing the better prospect. In our experiment choosing the chance device is equivalent to receiving the ambiguous or the risky prospect with equal probability. This implies that the chance device is chosen only when the utility difference between the two prospects is sufficiently small. However, when this is the case, the three available options (the risky prospect, the ambiguous one and the chance device) are equally attractive and should be chosen with equal probability. Given that the chance device is chosen almost exclusively in consecutive choice situations, our data do not seem consistent with a probabilistic choice model. In order to make this argument more robust we run an experiment where subjects had to make a series of choices between risky prospects and a sure amount. The risky prospects are exactly the same as those in the decision table of part 1 , while the sure amount is fixed in every choice situation and is equal to $€ 7.50$. As in the first experiment, in each situation subjects can have the chance device selecting one of the two options; the experimental

\footnotetext{
${ }^{11}$ The estimates of $\alpha$ indicate moderate concavity of the value function, in accord with the common hypothesis that utility is almost linear for small stakes. The estimated $\alpha$ of indecisive subjects is slightly higher, but not significantly different, than that of decisive subjects (0.91 and 0.84 respectively, Mann Whitney test $p=0.19$ ).
} 
procedures and all the other parts of the experiment are the same as in our first experiment. If repeated choice of the chance device has to be ascribed to a probabilistic choice process, we would expect that subjects also choose the chance device in a number of decision situations in this experiment. Indeed, given that the sure amount lies between the two possible outcomes of the lottery (which are $€ 0$ and $€ 15$ ), theoretically for each subject there exists at least one situation where the utility difference between the risky option and the sure amount is very small. 50 subjects participated in this experiment; we exclude from the analysis 4 subjects who violated monotonicity. Figure 2.3 shows the relative frequency of choices in favor of the chance device.

Figure 2.4: Relative frequency of indecisive choices under risk.

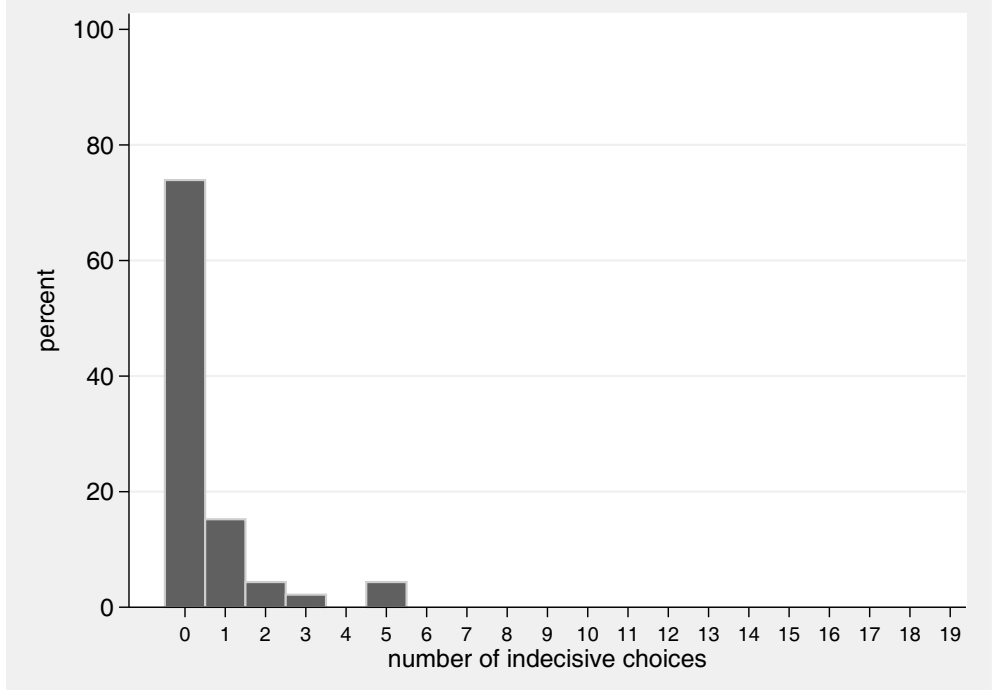

It appears that only $6.5 \%$ of the subjects choose the chance device more than twice, in contrast to the $49 \%$ share observed in the first experiment. This result clearly indicates that the high number of choices in favor of the chance device under ambiguity cannot be explained by arguments based on the existence of a small utility difference between prospects.

Regret aversion At last, we test whether Regret theory (Loomes and Sugden, 1982) can explain the repeated preference for the chance device. 
Regret theory predicts that between two options a DM chooses the one that maximizes a modified utility function which, for each state of nature, compares alternatives in terms of their outcomes and potential regret/rejoice feelings. Consider, for instance, choosing between two options, A and B, when only two states of the world are possible. If in a given state option A yields a more desirable consequence than option $B$, the individual anticipates that he may feel regret by choosing $B$ : he may reflect on how his position would have been had he chosen option A, and this thought may reduce the utility that he derives from the outcome of option B. Conversely, if in another state of nature option B yields the more desirable consequence, by choosing $\mathrm{B}$ the DM may experience what has been called rejoicing, that is extra pleasure associated with knowing that the best option has been chosen. The DM will ultimately choose B if its modified utility, that is B's utility plus the anticipated rejoice and minus the anticipated regret, is larger than A's modified utility, which is calculated in the same way.

Several scholars suggested that the chance device may be chosen in order to avoid regret feelings that can arise when actively choosing between the ambiguous and the risky prospect. First of all, we would like to point out that this interpretation may have some explanatory power only if we are ready to assume that anticipated feelings can exclusively arise when choosing between prospects and not when selecting the chance device. Indeed, if anticipated feelings would also be experienced when selecting the chance device, Regret theory would predict that the chance device is chosen only when the winning probability of the risky prospect is equal to the prior belief on the ambiguous event. ${ }^{12}$ The intuition behind this prediction is that all the three choice options in our experiment are characterized by the same potential outcomes which implies that only outcomes' likelihoods matter for decision making. Second, we need an additional assumption for Regret theory to be able to explain our data, namely that regret and rejoice are not equally strong feelings. In the appendix we formally show that if both assumption 1 (only active choices generate anticipated feelings) and assumption 2 (regret and rejoice are not equally strong feelings) hold then Regret theory may explain repeated choice of the chance device. In particular, the chance device

\footnotetext{
${ }^{12}$ Regret theory does not explicitly model decision making when probabilities are unknown: here we assume that subjects hold a unique prior belief on the ambiguous event.
} 
would be chosen more often the stronger the difference between regret and rejoice feelings. In the appendix we also show that under these assumptions, Regret theory would predict that the chance device is repeatedly selected when the choice problem involves comparing a risky prospect to a safe payment, like in the experiment described in the previous paragraph. However, Figure 2.3 shows that indecisive choice are hardly observed when subjects face choices between risky prospects and safe options. We conclude that Regret theory can only explain repeated choice of the chance device when ad hoc assumptions are made and, more importantly, only if we restrict our attention to choices between ambiguous and risky prospects.

Result 2.3.2. Several normative and descriptive models of decision making under uncertainty fail to account for repeated choice of the chance device.

\subsubsection{Are preferences of indecisive subjects incomplete?}

Our results show that approximately $50 \%$ of the subjects appear to be indecisive between risky and ambiguous prospects in at least three choice situations. Repeated choice of the chance device is inconsistent with the predictions of many decision making models: we have shown that neither normative models of decision making nor descriptive ones can account for it. In general, our results suggests that people may not always hold preferences for one source of uncertainty over another, even when they compare a familiar source, the risky prospect, with a less familiar one, the ambiguous prospect. This suggests that subjects repeatedly choosing the chance device may have incomplete preferences over uncertain prospects. Here we focus on models that allow preferences between two options to be incomplete when the DM holds multiple priors about an uncertain event, her utility function being well defined (Bewley, 2002 and Gilboa et al., 2010).

Consider Gilboa et al. (2010) decision making model. The authors introduce a preference relation that satisfies some basic conditions ${ }^{13}$, plus Independence and C-Completeness. The latter condition verifies that preferences between risky acts are complete; incompleteness may only arise when

\footnotetext{
${ }^{13}$ The basic conditions of the preference relation are reflexivity, transitivity, monotonicity, Archimedean continuity and non triviality.
} 
the DM has multiple prior beliefs about an ambiguous act. The authors then prove (Theorem 1) that such a preference relation is equivalent to the following:

$$
x \geq y \Leftrightarrow E_{p}(x) \geqslant E_{p}(y) \quad \forall p \in C
$$

That is, an act $x$ is weakly preferred over an act $y$ if and only if the expected utility of act $x$ is at least as large as the expected utility of act $y$ for each and every prior belief $p$ belonging to the closed and convex set of priors $C$. An act is strictly preferred to the other if it is in expectation always as good as the other and larger than the other according to at least one prior belief. Indifference holds when both acts are in expectation equal according to each and every prior belief. Conversely, a preference relation is incomplete whenever according to some beliefs $x$ yields a higher expected utility than $y$ and according to other beliefs $y$ yields a higher utility than $x$.

In our experiment, the acts $x$ and $y$ represent the choice to bet on the risky and ambiguous urn respectively. Thus, $E_{p}(y)$ is the expected utility of $y$ relative to a certain prior $p$. Consider, for instance, that one believes that the probability of drawing a winning ball from the ambiguous urn can take any value between 0.3 and 0.65 , inclusive. In the choice situation where the winning probability of the risky prospect is 0.3 the ambiguous prospect is in expectation at least as good as the risky one. Thus, the preference relation between prospects is complete, the ambiguous prospect being strictly preferred to the risky one. Consider now the consecutive choice situation which entails a risky prospect with a winning probability of 0.35 . In this case, the ambiguous prospect is at least as good as the risky prospect according to all prior beliefs but one, for which the expected utility of the ambiguous prospect is lower than that of the risky one. It follows that the preference relation is now incomplete. As a matter of fact, preferences may be incomplete in each and every choice situation where the winning probability of the risky urn lies within one's interval of prior beliefs.

The idea that repeated choice of the chance device follows from incomplete preference relations is a hypothesis that needs to be backed by further evidence. A problematic aspect is that subjects have to make choices in experiments and hence incomplete preferences are never fully observ- 
able. However, psychological and decision making literature suggest that choosing the chance device may be optimal when preferences are incomplete. People like to be able to justify their choices, to themselves and to others (Simonson, 1989). This need may be explained with several motives, like anticipation of possible regret feelings (Bell, 1982), cognitive dissonance (Festinger, 1957) or the anticipation that others will be observing the decisions made (Lerner and Tetlock, 1999). When preferences are incomplete, there is obviously no good reason to choose one prospect or the other: any choice would be difficult to justify. Thus, it seems conceivable that the random device becomes an appealing option.

In the next paragraph we propose an experiment to test whether the choice behavior of indecisive subjects is consistent with an incomplete preference relation.

\subsection{An experiment to test the completeness of preferences}

The purpose of our second experiment is to test whether repeated choice of the chance device is consistent with the potentially incomplete preference relation described in Gilboa et al. (2010). To this end, we increase the prize of the ambiguous prospect by a positive amount, while holding everything else the same as in the first experiment. We assume that on average subjects' prior beliefs on the composition of the ambiguous urn do not differ in the two experiments. ${ }^{14}$ Hence, for any belief on the ambiguous urn, the ambiguous prospect in experiment 2 has a higher expected value compared to experiment 1 . If people choose the chance device when preferences are incomplete, the situations where the chance device is mostly chosen should be different in the two experiments. In particular, in experiment 2 such choice situations should entail risky prospects with higher winning odds compared to those in experiment 1 . In what follows the argument is formally developed.

Consider the 21 choice situations in the first part of the experiment. We

\footnotetext{
${ }^{14}$ The elicitation of subjects' beliefs confirms that on aggregate the distribution of priors is not significantly different in the two experiments (Kolmogorov Smirnov test $p=0.74$ ).
} 
of preferences

indicate with $p_{n}$ the winning probability of the risky prospect in choice situation $n$ where $p_{1}=1$ and $p_{21}=0$ (choices are recoded as if red were to be the winning color for all subjects). Recall that according to (2.1) a subject may have incomplete preferences whenever $p_{n}$ lies strictly within her interval of prior beliefs about the winning probability of the ambiguous urn. A subject's worst prior for the composition of the ambiguous urn is defined as $w=p_{l}-\varepsilon$ where $p_{l}$ is the lowest $p_{n}$ among the situations where the random device is chosen and $0<\varepsilon<0.05$. We refer to $p_{l}$ as the lower bound of the indecisiveness interval. Similarly, $b=p_{h}+\varepsilon$ is the subject's best prior for the composition of the ambiguous urn, where $p_{h}$ is the highest $p_{n}$ among the situations where the random device is chosen. We refer to $p_{h}$ as the upper bound of the indecisiveness interval. The prospects positive outcome is $x_{1}$, while the zero outcome is $x_{2}$. Suppose that we want to induce complete preferences for those choice situations characterized by a $p_{n} \in\left[p_{l} ; \tilde{p}\right]$, with $\widetilde{p} \in\left[p_{l} ; p_{h}\right]$, the latter being the interval where a subject chooses the chance device. To induce complete preferences in the interval $\left[p_{l} ; \widetilde{p}\right]$, the prize increase $x$ that has to be added to the regular prize $x_{1}$ has to satisfy:

$$
w \times U\left(x_{1}+x\right)+(1-w) \times U\left(x_{2}\right) \geq \widetilde{p} \times U\left(x_{1}\right)+(1-\widetilde{p}) \times U\left(x_{2}\right)
$$

That is, the expected utility of the ambiguous prospects according to the subject's worst prior should be at least as large as the expected utility of the risky prospect characterized by a winning probability equal to $\widetilde{p}$.

Given the stakes employed in the experiment, we can make the simplifying assumption that utility is linear, that is $U(x)=x$. Since $x_{2}=0$ it follows that:

$$
x \geq \frac{(\widetilde{p}-w)}{w} x_{1}
$$

In addition, $x$ should be such that choices in favor of the chance device are not completely crowded out when the winning probability of the risky urn is larger than $\widetilde{p}$. To guarantee this $x$ has to satisfy:

$$
w \times\left(x_{1}+x\right)+(1-w) \times x_{2}<(\widetilde{p}+\varepsilon) \times x_{1}+(1-\widetilde{p}-\varepsilon) \times x_{2}
$$

That is, the expected utility of the ambiguous prospects according to the worst prior, $w$, should be smaller than the expected utility of the risky 
prospect characterized by a winning probability slightly larger than $\widetilde{p}$. This reduces to:

$$
x<\frac{(\widetilde{p}-w+\varepsilon)}{w} x_{1}
$$

From (2.3) and (2.5) it is easy to see that $x=\frac{(\widetilde{p}-w)}{w} x_{1}$ satisfies both conditions.

In sum, a subject's preferences shall become complete following an appropriate increase in the prize associated to the ambiguous prospect. Notice that subjects participating in experiment 1 do not take part in experiment 2. Hence, in experiment 2 we set a prize increase $x$ that is equal for all subjects and is based on the choices of participants in experiment 1 . Recall that in experiment 1 choices in favor of the chance device are most frequent when $p_{n} \in[0.35 ; 0.5]$. Also recall that $x_{1}=€ 15$ and $x_{2}=€ 0$. It follows that in order to increase the number of subjects having complete preferences in choice situations characterized by $p_{n} \in[0.35 ; 0.45]$, in experiment 2 we have to increase the $€ 15$ prize by $x=€ 5$.

By setting $x=€ 5$ we can make the following predictions: i) The chance device is chosen less often in experiment 2 compared to experiment 1 in all those choice situations where $0.35 \leq p_{n} \leq 0.45$. On the other hand, the chance device is chosen more often in experiment 2 than in experiment 1 in the choice situations characterized by $0.50 \leq p_{n} \leq 0.65$. ii) Subjects in experiment 2 are characterized by higher values of $p_{l}$ compared to subjects in experiment 1. iii) Subjects in experiment 2 are characterized by higher values of $p_{h}$ compared to subjects in experiment 1 . iv) Overall, the chance device is chosen less often in experiment 2 than in experiment 1 because the prize increase may be sufficiently high to make preferences of some subjects, those with a small interval of priors, always complete.

To summarize, experiment 2 presents subjects with the same series of 21 choice situations as in experiment 1 , with the only difference that the prize attached to the ambiguous prospect is now $€ 20$. All the other aspects of the experiment are exactly the same as in experiment 1.53 students from Maastricht University participated in the experiment which was conducted in March 2009. 78\% of the subjects were enrolled in the Faculty of Economics and Business Administration and $49 \%$ of them were male. The average age 
of preferences

was 25 years. The experiment lasted on average 90 minutes and the average earnings per subjects were $€ 28.7$.

\subsubsection{Results}

The choices of subjects participating in experiment 2 show that results obtained in experiment 1 are robust. ${ }^{15}$ First of all, the number of choices in favor of the risky prospect are significantly larger than $50 \%$ in all choice situations where $p_{n} \geq 0.55$ (binomial test $\mathrm{p}=0.01$ ). Conversely, choices in favor of the ambiguous prospect are significantly larger than $50 \%$ in all choice situations where $p_{n} \leq 0.45$ (binomial test $\mathrm{p}=0.01$ ). When the winning probability of the risky prospect equals $50 \%$ the number of choices in favor of the risky urn is not significantly different than the number of choices in favor of the ambiguous urn (binomial test $\mathrm{p}=0.19$ ). Thus, we can conclude that subjects in this experiment are moderately averse to ambiguity.

Figure 2.5 shows a histogram of the relative frequency of choices in favor of the chance device in experiment 2 . Notice that a large number of subjects repeatedly choose the chance device. Indeed, $60 \%$ of the subjects fall in the category of decisive subjects, while the remaining $40 \%$ choose the chance device in at least three choice situations. Hence, this experiment confirms that repeated choice of the chance device is a robust anomaly.

We now move on to compare the results of the two experiments. Table 2.2 shows data on the comparison of subjects' choices in the first part of the experiments.

Table 2.2: Intervals of indecisive choices.

\begin{tabular}{|c|c|c|}
\hline & experiment 1 & experiment 2 \\
\hline mean $p_{l}$ & 0.33 & 0.40 \\
\hline mean $p_{h}$ & 0.60 & 0.54 \\
\hline indecisive choices per subject & 3.7 & 2.5 \\
\hline
\end{tabular}

We find that in experiment 2 starting from the situation where $p_{n}=0.45$

\footnotetext{
${ }^{15}$ We exclude from the analysis 5 subjects who switched back and forth between the three options more than 4 times, thus displaying too noisy behavior. We also had to exclude 3 subjects that made highly inconsistent choices in the second part of the experiment.
} 
Figure 2.5: Relative frequency of indecisive choices.

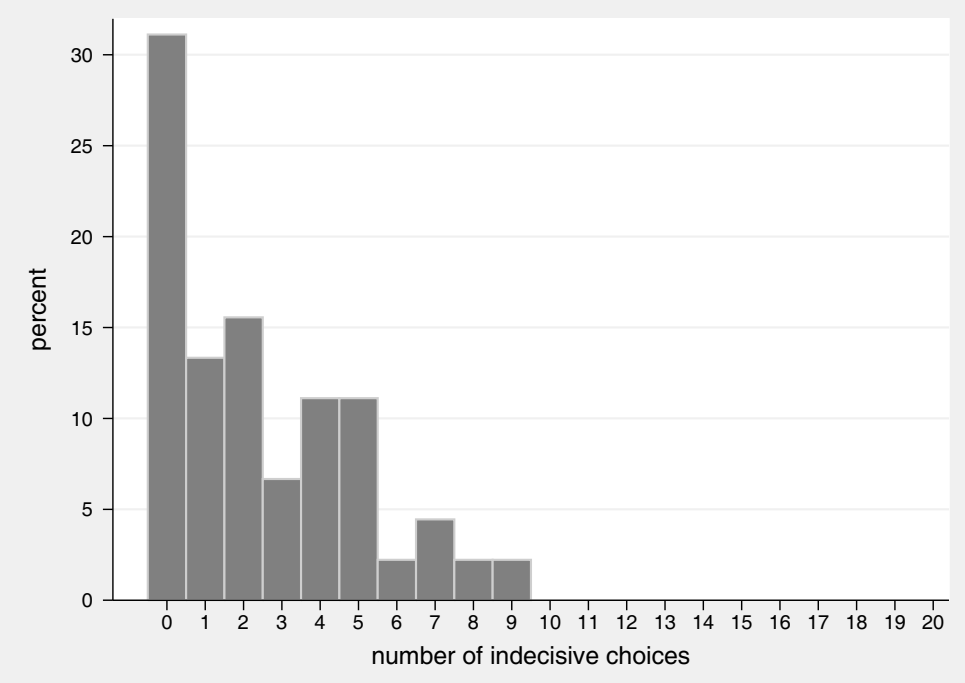

choices in favor of the chance device are less frequent compared to experiment 1 . On the other hand, the relative frequency of choices in favor of the chance device is higher in experiment 2 for those situations characterized by $0.5 \leq p_{n}<0.65$. Choices in favor of the chance device are less frequent in experiment 2 when $p_{n}=0.65$, a results that may be explained by the fact that fewer indecisive choices are predicted in experiment 2 (see point iv). In sum, the theoretical predictions in i) hold true.

In order to test the validity of theoretical prediction ii) we look at the lower bound of the indecisiveness interval. In experiment $1 p_{l}=0.33$ on average, while in experiment $2 p_{l}=0.40$ on average; a one tailed Mann Whitney test shows that subjects in experiment 2 are characterized by a significantly higher value of $p_{l}(\mathrm{p}=0.04) .{ }^{16}$ The second theoretical prediction ii) relates to the interval upper bound. In experiment $1 p_{h}=0.6$ on average, while in experiment $2 p_{h}=0.54$ on average; a one tailed Mann Whitney test shows that values of $p_{h}$ are not significantly different in the two experiments $(\mathrm{p}=0.46)$.

\footnotetext{
${ }^{16}$ If we exclude from both experiments those subjects who do not have connected intervals results are qualitatively unchanged but statistically insignificant.
} 
This result is actually not surprising because, as already mentioned, the prize increase may have been high enough to make preferences of some subjects complete. Indeed, the percentage of subjects who never choose the chance device increases from $24.5 \%$ in experiment 1 to $31 \%$ in experiment 2. Overall, the chance device is chosen less often in experiment 2 compared to experiment 1 , though this difference is not significant (one tailed Mann Whitney test $p=0.22$ ).

Result 2.4.1. In experiment 2 choices in favor of the chance device are distributed differently over decision situations compared to experiment 1. The change in the distribution is consistent with the existence of an incomplete preference relation between prospects.

Experiment 2 confirms that a large fraction of subjects repeatedly choose the chance device instead of selecting one prospect. Furthermore, it shows that choices of these subjects are consistent with the existence of an incomplete preference relation due to uncertainty. In the next section we investigate whether indecisiveness is related to individual characteristics of the decision maker.

\subsection{Individual characteristics}

Table 2.3 shows descriptive statistics on the relation between individual characteristics and indecisiveness for both experiments. First of all note that the level of experience with laboratory experiments, measured as the mean number of attended experiments, is not significantly different for indecisive subjects and decisive ones. The two groups are also not significantly different in terms of their performance in the CRT, measured as the number of correct answers. The parameters' values of the utility and probability weighting function are also not significantly different between indecisive subjects and decisive ones. These results are particularly interesting when compared to recent findings on the relation between cognitive abilities and risk attitude (Frederick, 2005 and Dohmen et al., 2010). Compared to subjects with low cognitive abilities, subjects with high cognitive abilities have been found to be less risk averse for small stakes. Our data show that neither risk attitude nor cognitive ability are systematically related to indeci- 
siveness in choice under uncertainty. One can speculate that smart subjects may appear more rational in experiments on decision making under risk because being rational in those experiments requires the ability to deal with numbers. On the contrary, in most real life situations and in our experiment as well, probabilistic information is not available. In such situations, cognitive ability may not always help to make decisions consistent with the axioms of rational choice.

Table 2.3: Individual characteristics, standard deviations in parenthesis.

\begin{tabular}{lllll}
\hline \hline & \multicolumn{2}{l}{ experiment 1} & \multicolumn{2}{l}{ experiment 2} \\
\cline { 2 - 5 } & decisive & indecisive & decisive & indecisive \\
\hline mean of attended experiments & $2.7(1.9)$ & $2.3(1.87)$ & $2.5(1.78)$ & $2.2(1.89)$ \\
median $\alpha$ & $0.82(0.19)$ & $0.87(0.2)$ & $0.62(0.16)$ & $0.68(0.12)$ \\
median $\gamma$ & $0.62(0.18)$ & $0.52(0.3)$ & $0.61(0.11)$ & $0.56(0.13)$ \\
mean CRT score & $1.63(1)$ & $1.57(0.99)$ & $1.74(1.1)$ & $1.61(1.14)$ \\
mean NFC & $3.88(0.59)$ & $3.73(0.66)$ & $3.77(0.52)$ & $3.67(0.73)$ \\
mean FI* & $3.3(0.67)$ & $3.61(0.57)$ & $3.52(0.41)$ & $3.44(0.51)$ \\
gender** (\% of male subjects) & 74 & 42 & 63 & 27 \\
\hline
\end{tabular}

Notes. **Significance at the $5 \%$ level, *Significance at the $10 \%$ level, Mann Whitney test two sided.

Recall that the Rational-Experiential Inventory consists of two scales: the Need for Cognition scale (NFC) and the Faith in Intuition scale (FI) which measure respectively analytical and intuitive processing. After dropping the items that load less than 0.3 on the respective factor, both scales are constituted of 11 items and are highly reliable (Cronbach's $\alpha=0.82$ for NFC, and $\alpha=0.79$ for FI). Furthermore, NFC and FI are not significantly related to each other (correlation coefficient $r=-0.13$ ), which means that analytical and intuitive processing are two independent constructs. Hence, for each subject we can compute the NFC and the FI score as the mean of the answers to the 11 items (recall that answers are on a 5 points scale). Given that repeated choice of the chance device violates rational choice, we expect subjects who are prone to it to be more inclined to think in an intuitive manner, rather than in a rational-analytical one. Indeed, indecisive 
subjects score slightly higher on the NFC scale but this difference is not significant. Indecisive subjects are also characterized by a lower score on the FI scale, but this difference is significant only in experiment 1 . In both experiments we observe that the group of decisive subjects is mainly composed by male subjects. Indeed, gender appears to be the only variable which is significantly different between indecisive and decisive subjects. Table 2.4 shows the results of a logit regression analysis.

Table 2.4: Explaining indecisiveness, logit regression results.

\begin{tabular}{lccc}
\hline \hline & \multicolumn{2}{c}{ dependent variable: probability of being indecisive } & \\
\cline { 2 - 4 } independent variables & coefficient & standard error & p-value \\
\hline attended experiments & -0.13 & 0.12 & 0.27 \\
$\alpha$ & 1.85 & 1.16 & 0.11 \\
$\gamma$ & 1.43 & 1.35 & 0.29 \\
CRT score & 0.01 & 0.22 & 0.98 \\
NFC score & -0.36 & 0.38 & 0.35 \\
FI score & 0.06 & 0.42 & 0.89 \\
male & -1.52 & 0.52 & 0.03 \\
constant & -2.56 & 2.5 & 0.9 \\
\hline observations & 98 & & \\
Log likelihood & -59.2 & & \\
likelihood ratio chi-square & 16.5 & & \\
p-value & 0.02 & & \\
\hline
\end{tabular}

The probability of being a indecisive is regressed on subject's individual characteristics. Since subjects in the two experiments are not significantly different, we pool data from the two experiments. ${ }^{17}$ Note that only the coefficient of gender is significant and negative, meaning that being male reduces the probability of being indecisive. Though not significantly, a higher need for cognition negatively affects the probability of being indecisive, whereas

\footnotetext{
${ }^{17}$ In fact, subjects in experiment 2 are characterized by a lower value of $\alpha$. Nevertheless, aggregation is warranted because the coefficient of the variable interacting a dummy for the experiment with $\alpha$ is not significant.
} 
a higher faith in intuition increases the probability of choosing the chance device. Also more experience with laboratory experiments negatively affects the probability of being indecisive though not significantly. Conversely, a higher value of $\gamma$, of $\alpha$ and a higher score in the CRT positively, but not significantly, affect the probability of being indecisive.

\subsection{Conclusions}

In this paper we experimentally study the behavior of subjects facing a series of choices between a risky and an ambiguous two-outcomes prospects. The possible outcomes and the level of ambiguity are the same in each choice situation, while risky prospects are characterized by different combinations of probability-outcomes. Our data show that half of the subjects in the experiment often choose a chance device to select between two prospects instead of actively picking one. The observed choice behavior is not consistent with the existence of a preference relation of true indifference, but rather indicates that subjects are indecisive when facing choices under uncertainty. Furthermore, the fact that subjects repeatedly choose the chance device constitutes a so far unexplored behavioral anomaly in decision making under uncertainty. Indeed, we have shown that both normative and descriptive models of decision making under uncertainty cannot account for it.

In our experiment, a DM with multiple prior beliefs on the ambiguous event is necessarily confronted with choice situations where her preferences may be incomplete. The larger the interval of prior beliefs, the more the choice situations where a DM may not have complete preferences. The observed behavioral anomaly seems consistent with the idea that a DM with incomplete preferences chooses the fair chance device. We use results from our first experiment to make choice predictions based on the incomplete preferences relation in Gilboa et al. (2010). Such predictions are then tested in a successive experiment: data are consistent with the hypothesis that avoidance of active decision making may follow from incomplete preference relations. Other anomalies, such as preference reversals (Eliaz and Ok, 2006) and the status quo bias (Mandler, 2004), have already been accounted by arguments based on incompleteness. Our results confirm that relaxing completeness may be a promising avenue in the development of descriptively valid deci- 
sion making models.

The results of our investigation offer as well interesting behavioral insights on choice behavior under uncertainty. The observed anomaly is in contrast with uncertainty aversion, which constitutes a well established empirical regularity. We suggest that uncertainty aversion may emerge in decision situations where people are "forced" to pick one of the available alternatives. On the other hand, when given a way out to the choice dilemma (the chance device in our case) many people do not actively select the more familiar risky prospects. Notably, in our experiment the chance device is neither a default option nor the status quo. Subjects explicitly choose a mechanism that decides instead of themselves.

In real life decision making is often performed under uncertainty. Financial investments, medical treatments and career choices for instance, are often chosen with a limited knowledge about the associated likelihood of success and failure. Our results suggest that in such decision situations many people may prefer not to choose by themselves when given the possibility to do so. Clearly, this behavior can have important economic consequences if not choosing comes at a cost for the decision maker; we believe that future research should be aimed at investigating this aspect.

\subsection{Appendix}

In the following we consider the discussed models of decision making under uncertainty and prove that they cannot account for the repeated avoidance of active choice.

\subsection{1 $\alpha$-maxmin Expected Utility Theory}

Consider $\alpha$ - maxmin Expected Utility Theory. The decision maker holds a set of prior $C=[\underline{c}, \bar{c}] \subseteq[0,1]$ on the ambiguous event and is characterized by an index $\alpha$ which captures attitude to ambiguity. The index varies from 0 to 1 and can be viewed as the weight that the decision maker places on the most pessimistic scenario, given his set of prior $C$. The utility function $U(\cdot)$ is the same assumed in expected utility theory. In our experiment, subjects would evaluate ambiguous prospects as follows: 


$$
\alpha \min _{q \in[\underline{c}, \bar{c}]}\left[q U\left(x_{1}\right)+(1-q) U\left(x_{2}\right)\right]+(1-\alpha) \max _{q \in[\underline{c}, \bar{c}]}\left[q U\left(x_{1}\right)+(1-q) U\left(x_{2}\right)\right]
$$

Where $q$ is the (unknown) winning probability of the ambiguous prospect, $x_{1}$ is the monetary prize equal to $€ 15$ and $x_{2}$ is the $€ 0$ outcome. Since the subject's worst prior is $\underline{c}$ and his best prior is $\bar{c}$ and $U\left(x_{2}\right)$ can be normalized to 0 , the above function can be written as:

$$
\alpha \underline{c} U\left(x_{1}\right)+(1-\alpha) \bar{c} U\left(x_{1}\right)
$$

If the decision maker chooses to delegate his decision in a given situation $n$, the lottery that assigns equal probability to the risky and to the ambiguous prospect is at least as good as the risky prospect characterized by a winning probability $p_{n}$ :

$$
\frac{1}{2}\left[\alpha \underline{c} U\left(x_{1}\right)+(1-\alpha) \bar{c} U\left(x_{1}\right)\right]+\frac{1}{2} p_{n} U\left(x_{1}\right) \geq p_{n} U\left(x_{1}\right)
$$

In addition he deems the lottery to be at least as good as the ambiguous prospect:

$$
\frac{1}{2}\left[\alpha \underline{c} U\left(x_{1}\right)+(1-\alpha) \bar{c} U\left(x_{1}\right)\right]+\frac{1}{2} p_{n} U\left(x_{1}\right) \geq \alpha \underline{c} U\left(x_{1}\right)+(1-\alpha) \bar{c} U\left(x_{1}\right)
$$

The above expressions can be rewritten as:

$$
\begin{aligned}
& \frac{1}{2}\left[\alpha \underline{c} U\left(x_{1}\right)+(1-\alpha) \bar{c} U\left(x_{1}\right)\right] \geq \frac{1}{2} p_{n} U\left(x_{1}\right) \\
& \frac{1}{2}\left[\alpha \underline{c} U\left(x_{1}\right)+(1-\alpha) \bar{c} U\left(x_{1}\right)\right] \leq \frac{1}{2} p_{n} U\left(x_{1}\right)
\end{aligned}
$$

It follows that:

$$
p_{n}=\alpha \underline{c}+(1-\alpha) \bar{c}
$$

Which means that $\alpha$-maxmin Expected Utility theory can account for indecisiveness only if $\underline{c}, \bar{c}$ and $\alpha$ change in each decision situation. 


\subsubsection{Cumulative Prospect Theory}

In order to apply CPT to choices in the table we need to recall that decision makers are assumed go through an initial editing phase. In this phase, complex choice problems are simplified by means of various routines (Starmer and Sugden, 1991). One of these routines is related to compound lotteries and leads to the so called isolation effect. If an individual is asked to make a choice between two lotteries, each of which is contingent on the occurrence of the same random event, then the choice is made as if that event is certain to occur. Assume that subjects employ this routine when evaluating prospects. When an indecisive choice is made the following equality holds:

$$
w^{+}\left(p_{n}\right) v\left(x_{1}\right)+\left(1-w^{+}\left(p_{n}\right)\right) v\left(x_{2}\right)=W^{+}(A) v\left(x_{1}\right)+\left(1-W^{+}(A)\right) v\left(x_{2}\right)
$$

where $v(\cdot)$ indicates the value function, $x_{1}$ is the monetary prize equal to $€ 15$ and $x_{2}$ is the $€ 0$ outcome. The letter $A$ indicates the event "drawing a winning ball from the ambiguous urn" and $p_{n}$ is the winning probability of the risky prospect in choice situation $n . w^{+}$and $W^{+}$are the weighting functions for risk and for uncertainty respectively. Since $v\left(x_{2}\right)$ can be normalized to 0 , from the equality above it follows that:

$$
W^{+}(A)=w^{+}\left(p_{n}\right)
$$

That is event $A$ has the same decision weight as probability $p_{n}$. Assume now that the decision maker delegates his decision between the prospects in the successive choice situation of the table. It follows that the weight of $p_{n+1}$ equals the weight given to event $A$. Since $W^{+}(A)$ should not change during the elicitation, the equality above implies that $w^{+}\left(p_{n}\right)=w^{+}\left(p_{n+1}\right)$. In general, if choices are delegated in $n$ consecutive decision situations in the table, it follows that:

$$
w^{+}\left(p_{n}\right)=w^{+}\left(p_{n+1}\right)=\ldots .,=w^{+}\left(p_{N}\right)
$$

which means that probabilities are equally wighted in all the decision situation where the decision maker appears to be indecisive. 


\subsubsection{Regret Aversion}

Consider the 21 decisions between the risky and the ambiguous prospects in the first experiment and assume that red is the winning color. In what follows the possible states of the world are listed, where $R_{R}$ means that a red ball is extracted from the risky urn, $R_{A}$ means that a red ball is extracted from the ambiguous urn, etc. $L_{R}$ means that the risky lottery matters for the payment of indecisive subjects, while $L_{A}$ means that the ambiguous lottery matters for the indecisive subjects.

$$
\begin{array}{ccc}
R_{R}, \stackrel{S}{L}_{R}, R_{A} & R_{R}, \stackrel{S_{2}}{L_{A}, R_{A}} B_{R}, \stackrel{S_{3}}{L_{R}, R_{A}} B_{R}, \stackrel{S_{4}}{L_{A}}, R_{A} \\
\stackrel{S_{5}}{B_{R}, B_{A}} & B_{R}, \stackrel{S_{6}}{L_{A}}, B_{A} R_{R}, \stackrel{S_{7}}{L_{R}, B_{A}} R_{R}, \stackrel{S_{8}}{L_{A}}, B_{A}
\end{array}
$$

We define: $p\left(S_{n}\right)$ : probability of state of the world $n$

$p_{n}$ : winning probability risky urn in decision situation $\mathrm{n}$

$q$ : winning probability ambiguous urn

$x_{1}$ : high prize, 15 Euro in the experiment

$x_{2}$ : low prize, 0 Euro in the experiment

$R(\cdot)$ : regret-rejoice function

Assume that in a given decision situation regret and rejoice can only be experienced when an active choice between the risky and the ambiguous prospect is made: choosing the chance device does not generate such feelings. Following Looms and Sugden (1982) the expected utility of the risky prospect is larger equal than the expected utility of the chance device, that is $E U($ risky) $\geq E U$ (device), if:

$$
\begin{array}{r}
p\left(S_{1}\right)\left[x_{1}-x_{1}+R\left(x_{1}-x_{1}\right)\right]+p\left(S_{2}\right)\left[x_{1}-x_{1}+R\left(x_{1}-x_{1}\right)\right]+p\left(S_{3}\right)\left[x_{2}-x_{2}+R\left(x_{2}-x_{2}\right)\right]+ \\
p\left(S_{4}\right)\left[x_{2}-x_{1}+R\left(x_{2}-x_{1}\right)\right]+p\left(S_{5}\right)\left[x_{2}-x_{2}+R\left(x_{2}-x_{2}\right)\right]+p\left(S_{6}\right)\left[x_{2}-x_{2}+R\left(x_{2}-x_{2}\right)\right]+ \\
p\left(S_{7}\right)\left[x_{1}-x_{1}+R\left(x_{1}-x_{1}\right)\right]+p\left(S_{8}\right)\left[x_{1}-x_{2}+R\left(x_{1}-x_{2}\right)\right] \geq 0 \\
\Leftrightarrow \\
p\left(S_{4}\right)\left[x_{2}-x_{1}+R\left(x_{2}-x_{1}\right)\right]+p\left(S_{8}\right)\left[x_{1}-x_{2}+R\left(x_{1}-x_{2}\right)\right] \geq 0
\end{array}
$$




$$
\begin{aligned}
& \left(1-p_{n}\right) \frac{1}{2} q\left[x_{2}-x_{1}+R\left(x_{2}-x_{1}\right)\right]+p_{n} \frac{1}{2}(1-q)\left[x_{1}-x_{2}+R\left(x_{1}-x_{2}\right)\right] \geq 0 \\
& \left(1-p_{n}\right) \frac{1}{2} q\left[-x_{1}+R\left(-x_{1}\right)\right]+p_{n} \frac{1}{2}(1-q)\left[x_{1}+R\left(x_{1}\right)\right] \geq 0 \\
& q\left[-x_{1}+R\left(-x_{1}\right)\right]-p_{n} q\left[-x_{1}+R\left(-x_{1}\right)\right]+p_{n}\left[x_{1}+R\left(x_{1}\right)\right]-p_{n} q\left[x_{1}+R\left(x_{1}\right)\right] \geq 0 \\
& q\left[-x_{1}+R\left(-x_{1}\right)-p_{n} R\left(-x_{1}\right)-p_{n} R\left(x_{1}\right)\right] \geq-p_{n} x_{1}-p_{n} R\left(x_{1}\right) \\
& q\left[x_{1}-R\left(-x_{1}\right)+p_{n} R\left(-x_{1}\right)+p_{n} R\left(x_{1}\right)\right] \leq p_{n}\left(x_{1}+R\left(x_{1}\right)\right) \\
& q \leq \frac{p_{n}\left(x_{1}+R\left(x_{1}\right)\right)}{x_{1}-R\left(-x_{1}\right)+p_{n} R\left(-x_{1}\right)+p_{n} R\left(x_{1}\right)}
\end{aligned}
$$

Further, the expected utility of the ambiguous prospect is larger equal than the expected utility of the chance device, that is $E U($ ambiguous $) \geq$ $E U($ device), if:

$$
\begin{array}{r}
p\left(S_{1}\right)\left[x_{1}-x_{1}+R\left(x_{1}-x_{1}\right)\right]+p\left(S_{2}\right)\left[x_{1}-x_{1}+R\left(x_{1}-x_{1}\right)\right]+p\left(S_{3}\right)\left[x_{1}-x_{2}+R\left(x_{1}-x_{2}\right)\right]+ \\
p\left(S_{4}\right)\left[x_{1}-x_{1}+R\left(x_{1}-x_{1}\right)\right]+p\left(S_{5}\right)\left[x_{2}-x_{2}+R\left(x_{2}-x_{2}\right)\right]+p\left(S_{6}\right)\left[x_{2}-x_{2}+R\left(x_{2}-x_{2}\right)\right]+ \\
p\left(S_{7}\right)\left[x_{2}-x_{1}+R\left(x_{2}-x_{1}\right)\right]+p\left(S_{8}\right)\left[x_{2}-x_{2}+R\left(x_{2}-x_{2}\right)\right] \geq 0 \\
\Leftrightarrow \\
p\left(S_{3}\right)\left[x_{1}-x_{2}+R\left(x_{1}-x_{2}\right)\right]+p\left(S_{7}\right)\left[x_{2}-x+R\left(x_{2}-x_{1}\right)\right] \\
\Leftrightarrow 0 \\
\Leftrightarrow \\
\left(1-p_{n}\right) \frac{1}{2} q\left[x_{1}-x_{2}+R\left(x_{1}-x_{2}\right)\right]+p_{n} \frac{1}{2}(1-q)\left[x_{2}-x+R\left(x_{2}-x_{1}\right)\right]
\end{array}
$$




$$
\begin{array}{r}
\left(1-p_{n}\right) \frac{1}{2} q\left[x_{1}+R\left(x_{1}\right)\right]+p_{n} \frac{1}{2}(1-q)\left[-x_{1}+R\left(-x_{1}\right)\right] \geq 0 \\
q\left[x_{1}+R\left(x_{1}\right)\right]-p_{n} q\left[x_{1}+R\left(x_{1}\right)\right]+p_{n}\left[-x_{1}+R\left(-x_{1}\right)\right]-p_{n} q\left[-x_{1}+R\left(-x_{1}\right)\right] \geq 0 \\
\Leftrightarrow \\
q\left[x_{1}+R\left(x_{1}\right)-p_{n} R\left(x_{1}\right)-p_{n} R\left(-x_{1}\right)\right] \geq p_{n} x_{1}-p_{n} R\left(-x_{1}\right) \\
\Leftrightarrow \\
q\left[x_{1}+R\left(x_{1}\right)-p_{n} R\left(x_{1}\right)-p_{n} R\left(-x_{1}\right)\right] \geq p_{n}\left(x_{1}-R\left(-x_{1}\right)\right) \\
\Leftrightarrow \\
q \geq \frac{p_{n}\left(x_{1}-R\left(-x_{1}\right)\right)}{x_{1}+R\left(x_{1}\right)-p_{n} R\left(x_{1}\right)-p_{n} R\left(-x_{1}\right)}
\end{array}
$$

If we assume that regret and rejoice are symmetric, that is $R(-x)=-R(x)$, conditions (2.6) and (2.7) imply that the chance device is chosen if $q \geq p_{n}$ and $q \leq p_{n}$. That is, the chance device is chosen only in the decision situation where $p_{n}=q$.

If we assume instead that regret and rejoice are not equally strong feelings, for example $|R(-x)|>R(x)$, conditions (2.6) and (2.7) imply that thick indecisiveness intervals can be justified by appropriate assumptions on the values of $R(-x)$ and $R(x)$. In particular, the stronger the difference between regret and rejoice feelings the larger the interval of indecisive choices.

We now consider our second experiment and test whether the observed shift in the indecisiveness interval is consistent with the existence of asymmetric regret and rejoice feelings.

We define:

$p\left(S_{n}\right)$ : probability of state of the world $n$

$p_{n}$ : winning probability risky urn in decision situation $n$

$q$ : winning probability ambiguous urn

$x_{1}$ : high outcome risky prospect, 15 Euro in the experiment

$x_{2}$ : low outcome, 0 Euro in the experiment 
$z$ : high outcome ambiguous prospect, 20 Euro in the experimentIn this experiment the expected utility of the risky prospect is larger equal than the expected utility of the chance device, that is $E U($ risky) $\geq E U$ (device), if:

$$
\begin{aligned}
& p\left(S_{1}\right)\left[x_{1}-x_{1}+R\left(x_{1}-x_{1}\right)\right]+p\left(S_{2}\right)\left[x_{1}-z+R\left(x_{1}-z\right)\right]+p\left(S_{3}\right)\left[x_{2}-x_{2}+R\left(x_{2}-x_{2}\right)\right]+ \\
& p\left(S_{4}\right)\left[x_{2}-z+R\left(x_{2}-z\right)\right]+p\left(S_{5}\right)\left[x_{2}-x_{2}+R\left(x_{2}-x_{2}\right)\right]+p\left(S_{6}\right)\left[x_{2}-x_{2}+R\left(x_{2}-x_{2}\right)\right]+ \\
& p\left(S_{7}\right)\left[x_{1}-x_{1}+R\left(x_{1}-x_{1}\right)\right]+p\left(S_{8}\right)\left[x_{1}-x_{2}+R\left(x_{1}-x_{2}\right)\right] \geq 0 \\
& p\left(S_{2}\right)\left[x_{1}-z+R\left(x_{1}-z\right)\right]+p\left(S_{4}\right)\left[x_{2}-z+R\left(x_{2}-z\right)\right]+p\left(S_{8}\right)\left[x_{1}-x_{2}+R\left(x_{1}-x_{2}\right)\right] \geq 0 \\
& p_{n} \frac{1}{2} q\left[x_{1}-z+R\left(x_{1}-z\right)\right]+\left(1-p_{n}\right) \frac{1}{2} q\left[x_{2}-z+R\left(x_{2}-z\right)\right]+ \\
& p_{n} \frac{1}{2}(1-q)\left[x_{1}-x_{2}+R\left(x_{1}-x_{2}\right)\right] \geq 0 \\
& p_{n} \frac{1}{2} q\left[x_{1}-z+R\left(x_{1}-z\right)\right]+\left(1-p_{n}\right) \frac{1}{2} q[-z+R(-z)]+p_{n} \frac{1}{2}(1-q)\left[x_{1}+R\left(x_{1}\right)\right] \geq 0 \\
& p_{n} q\left[x_{1}-z+R\left(x_{1}-z\right)\right]+q[-z+R(-z)]-p_{n} q[-z+R(-z)]+p_{n}\left[x_{1}+R\left(x_{1}\right)\right] \\
& -p_{n} q\left[x_{1}+R\left(x_{1}\right)\right] \geq 0 \\
& q p_{n}\left[x_{1}-z+R\left(x_{1}-z\right)\right]-z+R(-z)-p_{n}[-z+R(-z)]-p_{n}\left[x_{1}+R\left(x_{1}\right)\right] \geq-p_{n}\left[x_{1}+R\left(x_{1}\right)\right] \\
& q \leq \frac{p_{n}\left(x_{1}+R\left(x_{1}\right)\right)}{p_{n} R\left(x_{1}-z\right)-z+R(-z)\left(1-p_{n}\right)-p_{n} R\left(x_{1}\right)}
\end{aligned}
$$

The expected utility of the ambiguous prospect is larger equal than the expected utility of the chance device, $E U$ (ambiguous) $\geq E U$ (device), if: 


$$
\begin{aligned}
& p\left(S_{1}\right)\left[z-x_{1}+R\left(z-x_{1}\right)\right]+p\left(S_{2}\right)[z-z+R(z-z)]+p\left(S_{3}\right)\left[z-x_{2}+R\left(z-x_{2}\right)\right]+ \\
& p\left(S_{4}\right)[z-z+R(z-z)]+p\left(S_{5}\right)\left[x_{2}-x_{2}+R\left(x_{2}-x_{2}\right)\right]+p\left(S_{6}\right)\left[x_{2}-x_{2}+R\left(x_{2}-x_{2}\right)\right]+ \\
& p\left(S_{7}\right)\left[x_{2}-x_{1}+R\left(x_{2}-x_{1}\right)\right]+p\left(S_{8}\right)\left[x_{2}-x_{2}+R\left(x_{2}-x_{2}\right)\right] \geq 0 \\
& p\left(S_{1}\right)\left[z-x_{1}+R\left(z-x_{1}\right)\right]+p\left(S_{3}\right)\left[z-x_{2}+R\left(z-x_{2}\right)\right]+p\left(S_{7}\right)\left[x_{2}-x_{1}+R\left(y-x_{1}\right)\right] \geq 0 \\
& p_{n} \frac{1}{2} q\left[z-x_{1}+R\left(z-x_{1}\right)\right]+\left(1-p_{n}\right) \frac{1}{2} q[z+R(z)]+p_{n} \frac{1}{2}(1-q)\left[-x_{1}+R\left(-x_{1}\right)\right] \geq 0 \\
& p_{n} q\left[z-x_{1}+R\left(z-x_{1}\right)\right]+q[z+R(z)]-p_{n} q[z+R(z)]+p_{n}\left[-x_{1}+R\left(-x_{1}\right)\right] \\
& -p_{n} q\left[-x_{1}+R\left(-x_{1}\right)\right] \geq 0 \\
& q p_{n}\left[z-x_{1}+R\left(z-x_{1}\right)\right]+[z+R(z)]-p_{n}[z+R(z)]-p_{n}\left[-x_{1}+R\left(-x_{1}\right)\right] \\
& \geq-p_{n}\left[-x_{1}+R\left(-x_{1}\right)\right] \\
& q \geq \frac{p_{n}\left(x_{1}-R\left(x_{1}\right)\right)}{p_{n} R\left(z-x_{1}\right)+z+R(z)\left(1-p_{n}\right)-p_{n} R\left(-x_{1}\right)}
\end{aligned}
$$

Conditions (2.8) and (2.9) imply that in a certain decision situation the chance device is the utility maximizing choice if the DM believes that $q$ takes a value in the interval of priors identified by the conditions. In fact, for an arbitrary value of $q$ the chance device is the optimal choice when the risky prospect is characterized by a higher winning likelihood as compared to the one which justifies choosing the chance device in the first experiment. Hence, indecisiveness in our experiments can be explained assuming that regret and rejoice are not equally strong feelings that are only experienced when making an active choice.

The validity of an explanation based on anticipated feelings can be further tested by considering choice in the experiment described at page 11 . 
Subjects made 21 decisions between varying risky lotteries and a fixed sure payment: the possible states of the world are listed below, where $R_{R}\left(B_{R}\right)$ means that a red (black) ball is extracted from the risky urn. The letter $R$ indicate that the risky prospect is relevant, while the letter $S$ indicates that the sure payment is relevant, for the payment of indecisive subjects. As before, we assume that red is the winning color. Since the high prize of the risky prospect is 15 Euro and the low prize is 0 Euro, we use the letters $x_{1}$ and $x_{2}$ as before. The sure payment always equals 7.5 Euro and we indicate it with $\frac{x_{1}}{2}$.

$$
\begin{array}{ccccc}
S_{R} & S_{2} & R_{R}, S & B_{R}^{S_{3}}, R & B_{R}^{S_{4}}, S
\end{array}
$$

The expected utility of the risky prospect is larger equal the expected utility of chance device, that is $E U($ risky) $\geq E U$ (device), if:

$$
\begin{gathered}
p\left(S_{1}\right)\left[x_{1}-x_{1}+R\left(x_{1}-x_{1}\right)\right]+p\left(S_{2}\right)\left[x_{1}-\frac{x_{1}}{2}+R\left(x_{1}-\frac{x_{1}}{2}\right)\right] \\
+p\left(S_{3}\right)\left[x_{2}-x_{2}+R\left(x_{2}-x_{2}\right)\right]+p\left(S_{4}\right)\left[x_{2}-\frac{x_{1}}{2}+R\left(x_{2}-\frac{x_{1}}{2}\right)\right] \geq 0 \\
p_{n} \frac{1}{2}\left[x_{1}-\frac{x_{1}}{2}+R\left(x_{1}-\frac{x_{1}}{2}\right)\right]+\left(1-p_{n}\right) \frac{1}{2}\left[-\frac{x_{1}}{2}+R\left(-\frac{x_{1}}{2}\right)\right] \geq 0 \\
p_{n}\left[\frac{x_{1}}{2}+R\left(\frac{x_{1}}{2}\right)+\frac{x_{1}}{2}-R\left(-\frac{x_{1}}{2}\right)\right] \geq \frac{x_{1}}{2}-R\left(-\frac{x_{1}}{2}\right) \\
\Leftrightarrow \\
p_{n}\left[x_{1}+R\left(\frac{x_{1}}{2}\right)-R\left(-\frac{x_{1}}{2}\right)\right] \geq \frac{x_{1}}{2}-R\left(-\frac{x_{1}}{2}\right) \\
\Leftrightarrow \\
p_{n} \frac{x_{1}}{x_{1}+R\left(\frac{x_{1}}{2}\right)-R\left(-\frac{x_{1}}{2}\right)}
\end{gathered}
$$

The expected utility of the safe payment is larger equal the expected utility of the chance device, that is $E U($ safe $) \geq E U$ (device), if: 


$$
\begin{array}{r}
p\left(S_{1}\right)\left[\frac{x_{1}}{2}-x_{1}+R\left(\frac{x_{1}}{2}-x_{1}\right)\right]+p\left(S_{2}\right)\left[\frac{x_{1}}{2}-\frac{x_{1}}{2}+R\left(\frac{x_{1}}{2}-\frac{x_{1}}{2}\right)\right] \\
+p\left(S_{3}\right)\left[\frac{x_{1}}{2}-x_{2}+R\left(\frac{x_{1}}{2}-x_{2}\right)\right]+p\left(S_{4}\right)\left[\frac{x_{1}}{2}-\frac{x_{1}}{2}+R\left(\frac{x_{1}}{2}-\frac{x_{1}}{2}\right)\right] \geq 0 \\
\Leftrightarrow \\
p_{n} \frac{1}{2}\left[\frac{x_{1}}{2}-x_{1}+R\left(\frac{x_{1}}{2}-x_{1}\right)\right]+\left(1-p_{n}\right) \frac{1}{2}\left[\frac{x_{1}}{2}+R\left(\frac{x_{1}}{2}\right)\right] \geq 0 \\
p_{n}\left[\frac{x_{1}}{2}-x_{1}+R\left(\frac{x_{1}}{2}-x_{1}\right)-\frac{x_{1}}{2}-R\left(\frac{x_{1}}{2}\right)\right] \geq-\left[\frac{x_{1}}{2}+R\left(\frac{x_{1}}{2}\right)\right] \\
\Leftrightarrow \\
p_{n}\left[-\frac{x_{1}}{2}+R\left(-\frac{x_{1}}{2}\right)-\frac{x_{1}}{2}-R\left(\frac{x_{1}}{2}\right)\right] \geq-\left[\frac{x_{1}}{2}+R\left(\frac{x_{1}}{2}\right)\right] \\
p_{n}\left[-x_{1}+R\left(-\frac{x_{1}}{2}\right)-R\left(\frac{x_{1}}{2}\right)\right] \geq-\left[\frac{x_{1}}{2}+R\left(\frac{x_{1}}{2}\right)\right] \\
\Leftrightarrow \\
p_{n}-R\left(-\frac{x_{1}}{2}\right)+R\left(\frac{x_{1}}{2}\right) \\
p_{n}\left[x_{1}-R\left(-\frac{x_{1}}{2}\right)+R\left(\frac{x_{1}}{2}\right)\right] \leq \frac{x_{1}}{2}+R\left(\frac{x_{1}}{2}\right)
\end{array}
$$

Condition (2.10) and (2.11) imply that we should observe large intervals of indecisive choices in this experiment. However, as this is not the case, we can at reject the hypothesis that indecisiveness is motivated by anticipated regret for active choices. 
2.7. Appendix

\subsubsection{Instructions of the experiment}

We report here the original instructions used in the first experiment and in brackets the parts changing in the experiment investigating delegation in risky choices. The instructions used in the second experiment are similar and available upon request. Notice that the instructions were computerized.

\section{Part 1}

Shortly you are going to face 21 choice situations (situations 1-21). These choice situations will involve two urns (i.e. boxes). These urns really exist and they will play an important role in determining your earnings. You might have seen them on the table when you entered the lab. At the end of the experiment you will have the possibility to personally check their content.

In one urn there are 100 balls colored black and red. The exact number of black and red balls contained in this urn is always displayed in the decision table that you will see shortly. For convenience we call this urn Urn A. The other urn, that we call Urn B, contains 100 balls as well. However, the exact number of black and red balls in this urn is unknown to you. In fact, the composition of Urn B is also unknown to us because it was composed by a colleague of us and sealed thereafter, while we were absent. Our colleague was free to put any number of red and/or black balls into this urn provided the total number of balls is 100 .

In each choice situation you will be asked to bet on a draw of a ball of a certain color by selecting one of the two different types of urns. You are first given the possibility to select the color (black or red) that you like to bet on. The color you select will neither be to your advantage nor to your disadvantage. Also note that you will choose the color once for all choice situations.

[Shortly you are going to face 21 choice situations (situations 1-21). These choice situations will involve one urn (i.e. a box). This urn really exists and it will play an important role in determining your earnings. In the urn there are 100 balls colored black and red. The exact number of black and red balls contained in the urn changes in each choice situation and is always displayed in the decision table that you will see shortly. In each choice situation you will be asked whether you want to bet on a draw of a ball of a 
certain color from the urn or whether you prefer to receive a certain amount of money. You are first given the possibility to select the color (black or red) that you like to bet on. The color you select will neither be to your advantage nor to your disadvantage. Also note that you will choose the color once for all choice situations.]

This is a screen shot of a part of the table you are going to see. Each row of the table represents one choice situation:

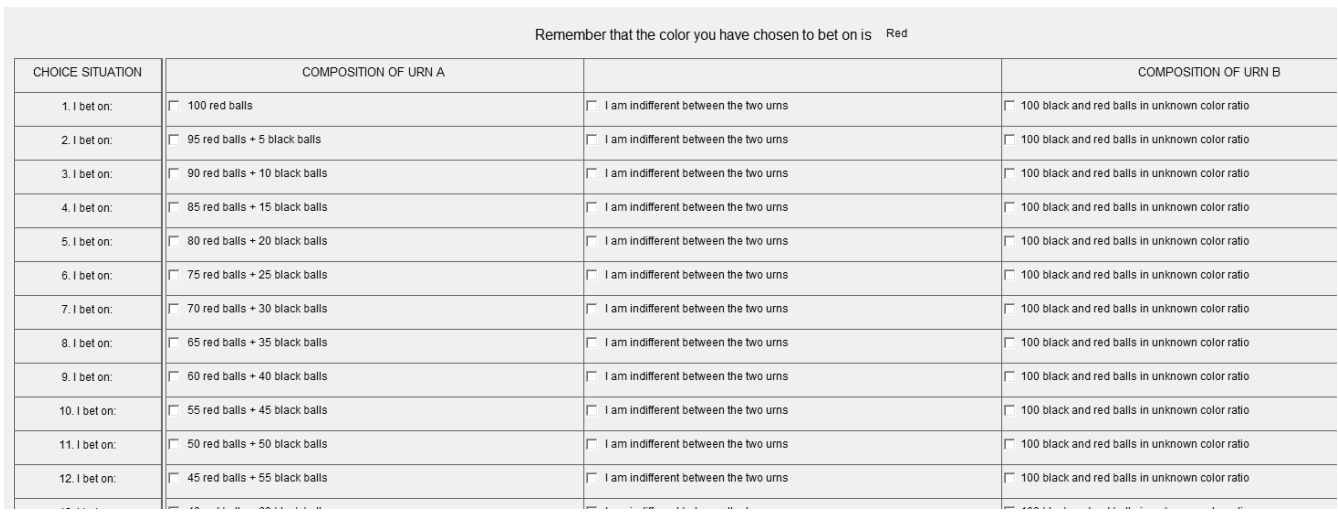

In each row you have to decide between Urn $\mathbf{A}$ and Urn $\mathbf{B}$ to bet on the color you have selected. You can also state that you are indifferent between the two urns.

Recall that Urn B contains an unknown proportion of 100 black and red balls. Urn A contains 100 balls as well: the proportion of black and red balls is always displayed in the table.

[In each row you have to decide whether you want to bet on the color you have selected or whether you want to receive 7.50 Euro for sure. You can also state that you are indifferent between these two options.

\section{Determination of earnings}

At the end of the experiment one of the choice situations in the table is randomly selected with equal probability to determine your earnings. Thereafter, a ball is drawn from the urn you decided to bet on in the choice situation that was randomly selected.

Suppose, for example, that red is your color and that choice situation 7 is 
randomly selected. Suppose further that you decided to bet on Urn A in that choice situation. At the end of the experiment, a ball is drawn from Urn A, which contains 70 red balls and 30 black balls in choice situation 7 . You receive 15 Euro if the ball is red and nothing otherwise.

Similarly, if in choice situation 7 you have decided to bet on Urn B, which contains 100 balls in unknown color composition, a ball is drawn from it. You receive 15 Euro if the ball is red and nothing otherwise. In case you were indifferent between the two urns, one is randomly selected with equal probability to determine your earnings.

[ At the end of the experiment one of the choice situations in the table is randomly selected with equal probability to determine your earnings. Depending on which choice situation is selected, the experimenter will put the appropriate number of red and black balls in the urn. For instance, if choice situation 12 is selected for payment, the experimenter will put 55 red balls and 45 black balls in the urn. At the end of the experiment you will have the possibility to personally check the content of the urn.

Suppose, for example, that you selected red and that choice situation 7 is randomly selected at the end of the experiment. Suppose further that you chose to bet on the urn in that choice situation. A ball is then drawn from the urn which contains 70 red balls and 30 black balls in situation 7 . You receive 15 Euro if the ball is red and nothing otherwise.

Differently, the ball drawn from the urn does not influence your earnings if in choice situation 7 you decided that you prefer to get 7.50 Euro for sure. In case you were indifferent between betting on the urn and earning 7.50 Euro for sure, one of these two options is randomly selected with equal probability to determine your earnings.

\section{Estimation of the composition of Urn B}

Now that you have made your choices, we would like to ask you for your best estimate of the color composition of Urn B.

The categories below are intervals indicating the number of red balls that might be contained in Urn B. Please click on the check box that represents your best estimate. You can also click on more than one box.

Consider the following random examples.

For instance, if you believe that there are between 12 and 34 red balls in 
Urn B, you should click on the 3rd, 4th, 5th 6 th and 7 th check box from the left.

For instance, if you believe that there are between 72 and 74 red balls in Urn B than you should click on the 15th check box form the left.

For instance, if you believe that there are exactly 6 red balls in Urn B than you should click on the 2nd check box from the left.

If you believe that there between 17 and 24 red balls or between 63 and 69 red balls in Urn B then you should click on the 4th, 5th, 13th and 14th check box. Notice that this part was not included in the experiment about delegation under risk.

\section{Part 2}

You are now going to make another series of choices. These choices will not influence your earnings from the choices you just made, nor will your earlier choices influence the earnings from the choices you are going to make. After you have made the these choices you will be asked to answer some questions. Thereafter the experiment will be over.

In the following, you will be confronted with a series of 33 decision situations that will appear in random order on the screen. All these decision situations are completely independent of each other. A choice you made in one decision situation does not affect any of the other following decision situations.

Each decision situation is displayed on a screen. The screen consists of 20 rows. You have to decide for every row whether you prefer option A or option B. Option A is a lottery and is the same for every row in a given decision situation, while the secure option B takes 20 different values, one for each row. By clicking on NEXT you will see an example screen of a decision situation.

This is a screen shot of one decision situation you are going to face. You are not asked to make choices now! Please have a careful look. Determination

\section{of earnings}

At the end of the experiment one of the 33 decision situations will be randomly selected with equal probability. Once the decision situation is selected, one of the 20 rows in this decision situation will be randomly selected 
2.7. Appendix

\begin{tabular}{|c|c|c|c|}
\hline DECISIOO Struation 13. & $\begin{array}{l}\text { OPIIONA } \\
\text { LOTERY }\end{array}$ & YOUR Сноске & $\begin{array}{l}\text { OPTIONB } \\
\text { sURE AMOUNT }\end{array}$ \\
\hline choice 1 & & $A \subset C B$ & 10. \\
\hline choice 2 & & $A \subset \subset B$ & 9.50 \\
\hline choice 3 & & $A \subset C B$ & 9. \\
\hline choice 4 & & $A C C B$ & 8.50 \\
\hline choice 5 & & $A \subset C B$ & 8.- \\
\hline choice 6 & & $A \subset C B$ & 7.50 \\
\hline choice 7 & $\begin{array}{l}\text { With } 55 \% \text { chance you receive } 10 \text {. Euro, } \\
\text { with } 45 \% \text { chance receive }\end{array}$ & $A \subset C B$ & 7.- \\
\hline choice 8 & & $A \subset \subset B$ & 6.50 \\
\hline choice 9 & & $A \subset C B$ & 6.- \\
\hline choice 10 & & $A C C B$ & 5.50 \\
\hline choice 11 & & $A \subset C B$ & 5.- \\
\hline choice 12 & & $A \subset C B$ & 4.50 \\
\hline choice 13 & & $A \subset \subset B$ & 4. \\
\hline choice 14 & & $A \subset \subset B$ & 3.50 \\
\hline choice 15 & & $A \subset C B$ & 3.- \\
\hline choice 16 & & $A \subset \subset B$ & 2.50 \\
\hline choice 17 & & $A \subset C B$ & 2.- \\
\hline choice 18 & & $A \subset C B$ & 1.50 \\
\hline choice 19 & & $A \subset C B$ & 1. \\
\hline choice 20 & & $A \subset C B$ & 0.50 \\
\hline
\end{tabular}

with equal probability.

The choice you have made in this specific row will determine your earnings. Consider, for instance, the screen shot that you have just seen.

Option A gives you a 55\% chance to earn 10.- Euro and a $45 \%$ chance to earn nothing. Option B is always a sure amount that ranges from 10.- Euro in the first row, to 0.50 Euro in the 20th row. Suppose that the 12th row is randomly selected. If you would have selected option $B$, you would receive 4.50 Euro. If, instead, you would have selected option A, the outcome of the lottery determines your earnings. The lottery will be paid out by publicly drawing a card from a stack of numbered cards.

Please note that each decision situation has the same likelihood to be the one that is relevant for your earnings. Therefore, you should view each decision independently and consider all your choices carefully.

\section{Part 3}

\section{Cognitive Reflection Test}

You have now finished with the 33 decision situations. In the following 
screens we ask you to answer some questions. Please read the following questions carefully and type your answer in the boxes. You will earn 0.50 Euro for each correct answer provided.

(1) A bat and a ball cost 1.10 Euro in total. The bat costs 1.00 Euro more than the ball. How many cents does the ball cost?

(2) If it takes 5 machines 5 minutes to make 5 widgets, how long (in minutes) would it take 100 machines to make 100 widgets?

(3) In a lake, there is a patch of lily pads. Every day, the patch doubles in size. If it takes 48 days for the patch to cover the entire lake, how many days would it take for the patch to cover half of the lake?

(4) Two cars are on a collision course, traveling towards each other in the same lane. Car A is traveling $70 \mathrm{~km}$ an hour. Car B is traveling $80 \mathrm{~km}$ an hour. How far apart are the cars one minute before they collide? Please answer in $\mathrm{km} .^{18}$

\section{Rational Experiential Inventory}

What is your opinion on the following statements?(subjects had to answer on a 5 point scale, where $1=$ "completely false"; $5=$ =completely true")

1. I would rather do something that requires little thought than something that is sure to challenge my thinking abilities

2. I don't like to have the responsibility of handling a situation that requires a lot of thinking.

3. I would prefer complex to simple problems.

4. I find little satisfaction in deliberating hard and for long hours.

5. Thinking is not my idea of fun.

6. The notion of thinking abstractly is not appealing to me.

7. I prefer my life to be filled with puzzles that I must solve.

8. Simply knowing the answer rather than understanding the reasons for

\footnotetext{
${ }^{18}$ This question is not part of the original CRT by Shane (2005). We added it to increase the complexity of the task. However, in the data analysis we do not consider answers to this question.
} 
the answer to a problem is fine with me.

9. I don't reason well under pressure.

10. The idea of relying on thought to make my way to the top does not appeal to me.

11. I prefer to talk about international problems rather than gossip about celebrities.

12. Learning new ways to think doesn't excite me very much.

13. I would prefer a task that is intellectual, difficult, and important to one that is somewhat important but does not require much thought.

14. I generally prefer to accept things as they are rather than to question them.

15. It is enough for me that something gets the job done, I don't care how or why it works.

16. I tend to set goals that can be accomplished only by expending considerable mental effort.

17. I have difficulty thinking in new and unfamiliar situations.

18. I feel relief rather than satisfaction after completing a task that required a lot of mental effort.

19. I try to anticipate and avoid situations where there is a likely chance I will have to think in depth about something.

20. My initial impressions of people are almost always right.

21. I trust my initial feelings about people.

22 . When it comes to trusting people, I can usually rely on my "gut feelings."

23. I believe in trusting my hunches.

24. I can usually feel when a person is right or wrong even if I can't explain how I know.

25. I am a very intuitive person.

26 . I can typically sense right away when a person is lying.

27. I am quick to form impressions about people.

28. I believe I can judge character pretty well from a person's appearance.

29. I often have clear visual images of things.

30. I have a very good sense of rhythm.

31. I am good at visualizing things. 



\section{Chapter 3}

\section{Fairness and Uncertainty}

\subsection{Introduction}

Resources often have to be (re)allocated without knowledge of the true state of nature or before the state of nature is known. In such situations the final position of the recipients depends on the allocation as well as on which one of the several uncertain states of the world materializes. The uncertainty about how the allocated resources translate into final positions complicates the definition of what constitutes a fair allocation in these distribution problems.

Consider, for instance, the income taxation of self employed workers, whose liabilities are usually calculated on the basis of self-reported income. In countries where treacherous declarations are difficult to detect or where tax evasion is frequent, the true redistributive effects of income taxation may be quite uncertain. The difficulty of finding a shared definition of fairness is effectively illustrated by the introduction of a new fiscal instrument by the Italian government in 1993. The instrument substitutes self-declarations of income with estimates based on the likelihood that each taxpayer belongs to a series of income groups: ${ }^{1}$ affected taxpayers strongly opposed the introduction of the new system, arguing that taxation based on probabilistic estimates is unfair.

Uncertainty about the true state of nature does not solely enter economic

\footnotetext{
${ }^{1}$ For detailed information consult http://www.agenziaentrate.gov.it
} 
problems. One of the main distributive justice criteria for organ transplant, the maximum benefit criteria, maintains ranking candidates according to their survival likelihood after the transplant. Opposers of the maximum benefit criteria argue that medical success is difficult to predict and hence that equal access should be granted to everybody (Childress, 2001). More generally, this example shows that people may disagree on what constitutes a fair allocation even when they do not have stakes in the distribution at hand.

In this paper we report the results of an experiment designed to investigate people's ideas about fairness in distribution problems where the state of nature is uncertain at the moment of the distribution decision. By uncovering the sources of people's disagreement about fairness, our investigation may help designing policies that are in line with generally shared fairness ideas. In the experiment participants are randomly divided into groups of three. In the first part of the experiment two group members work on a real effort task by which they earn money that is deposited in a joint account. The task is designed to minimize differences in productivity so that subjects can in principle acquire equal rights in the production phase (on the importance of earned rights and claims see, for example, Gächter and Riedl, 2005 and 2006). The third group member, the so called Benevolent Dictator (Konow, 2000 ), is then asked to allocate the money in the joint account between the two other group members, him/herself having no stakes in the account. This set up allows inferring people's distributive principles because it is designed in a way that the only possible concern for the Benevolent Dictator (BD) is to treat recipients fairly. Further, it has been shown that distributions by impartial third parties are much less sensitive to personal characteristics and expectations as compared to distributions decided behind the veil of ignorance (Aguiar et al., 2010). The BD has to make allocation decisions in several distribution problems knowing that at the end of the experiment one is randomly selected for the payment of the recipients. In all problems except one the final earnings of one of the recipients are uncertain and can be larger or smaller than the allocated amount, depending on the realized state after the distribution. Further, the level of uncertainty is systematically varied in the studied distribution problems in a way that the higher the potential final earnings the smaller their likelihood. The other recipient always earns 
exactly what is allocated to him/her. This design allows us investigating situations where recipients differ in their exposure to uncertainty and where the final distribution of outcomes can be more or less extreme. We only consider situations where uncertainty is independent from subjects' characteristics and cannot be influenced by subjects' decisions. Theoretically, three distributive justice principles are relevant: Equality, Utilitarianism and Equity are applied to our distribution problems in order to derive quantitative predictions on allocations. Predictions are then contrasted with actual data on allocations.

Traditionally, to empirically investigate people's distributive justice ideas researchers employed surveys and vignette studies (see, for example, Yaari and Bar-Hillel, 1984 and Kahneman et al., 1986) and only relatively recently incentivized experiments have been used. ${ }^{2}$ Konow (2000) first introduced the Benevolent Dictator game; in his experiment the BD was asked to allocate to two anonymous recipients the joint product of their work. The author finds that the product is less likely to be allocated in proportion to the individual contributions when differences in productivity are randomly assigned compared to when they result from subjects' differential efforts. These results have been replicated by Dickinson and Tiefenhalter (2002) who contrast earned rights to non-earned ones in a more complex environment. Cappelen et al. (2007) use allocation data from a standard dictator game to estimate the distribution of three principles of distributive justice in situations involving production. The authors adopt a random utility model that allows them disentangling dictators' allocation decisions into selfish motives and fairness principles. In another study Cappelen et al. (2011) investigate the distribution choices of both non-involved subjects and stakeholders in situations where inequalities in output are determined by risk taking behavior. The authors find that inequalities between risk takers and non-risk takers are mostly viewed as fair, but that inequalities between lucky and unlucky risk takers are instead eliminated.

To the best of our knowledge, we are the first investigating allocation problems where a division has to be made before the state of nature is known and where subjects cannot influence the level of uncertainty affecting their

\footnotetext{
${ }^{2}$ For empirical studies on distributive justice based on vignettes see also Schokkaert and Overlaet, 1989 and Faravelli, 2007.
} 
outcomes. Moreover, differently than in the reviewed studies, we do not focus on issues of merit but rather on fairness in situations where recipients differ due to factors beyond their direct influence.

We find that Benevolent Dictators agree on the equal split as the fair distribution, only when the final distribution of resources is certain. In contrast, when uncertainty is involved Benevolent Dictators differ substantially in their allocation decisions both within and across distribution problems. Since the perception of uncertainty is subjective and values often pluralistic, disagreement may stem from the fact that people hold different ideas about what is fair. Using a weighted distance criteria, we show that $50 \%$ of all the allocations are consistent with the principle of Equality, $17 \%$ with Utilitarianism and $33 \%$ with the Equity principle. We also observe that the interpretation of the Equality principle is context dependent as it is systematically related to the level of uncertainty of the distribution problem.

In the following section we discuss theories of distributive justice that are relevant for the distribution problems at hand and analytically derive the allocations predicted by these theories. In section 3.3 the experimental design is described in detail. In section 3.4 results are presented and discussed. In section 3.5 concluding remarks are drawn. In the Appendix details on the analytical derivation of allocations are provided.

\subsection{Distributive justice when outcomes are uncertain}

\subsubsection{The general problem}

Consider a distribution problem in which a certain amount $X$ of a resource has to be divided among a number of individuals $i$ in a normatively fair way. Individuals are equally deserving in the sense that all of them produce the same level of output under the same working conditions. The final distribution of resources depends on the state of nature which is uncertain at the moment of the division. After the division, uncertainty is resolved and the final distribution of resources is determined.

We indicate with $x_{i}$ the amount allocated to person $i$. The expected value of $x_{i}$ is given by the following expression: 


$$
E\left(x_{i}\right)=\sum_{e=1}^{n} p_{e} x_{i} k_{i, e} \text { with } \sum_{e=1}^{n} p_{e}=1
$$

where $0 \leq p_{e} \leq 1$ is the probability of event $e$ occurring and $k_{i, e} \in \mathbb{R}$ captures how event $e$ affects allocation $x_{i}$. For example, $k_{i, e}=1.5$ means that if event $e$ is realized the amount of resources of person $i$ increases by $50 \%$.

For the purpose of experimental implementation, we focus on a limited number of distribution problems $m \in\{1, . ., 7\}$ that are derived by introducing restrictions on the values of the variables in (3.1). In each studied distribution problem $m$ a monetary amount $X$ has to be divided between two persons called $\mathrm{R}$ (for exposed to risk) and $\mathrm{C}$ (for facing certain outcomes), such that $X=x_{R}^{m}+x_{C}^{m}$. We assume that only two states are possible, namely that event $e$ or its complement, $\bar{e}$, is realized. In order to simplify notation we indicate $k_{i, e}^{m}$ with $k_{i}^{m}$ and $k_{i, \bar{e}}^{m}$ with $\bar{k}_{i}^{m}$. Furthermore, assumptions on the values of $p_{e}^{m}$ and on $k_{i}^{m}, \bar{k}_{i}^{m}$ are made. First, we set $k_{C}^{m}=\bar{k}_{C}^{m}=1 \forall m$, which implies that person C's final earnings do not depend on which state is realized and are thus equal to what is allocated to her in all studied distribution problems. Second, we choose $k_{R}^{m}>1>\bar{k}_{R}^{m}$ and $p_{e}^{m}$ such that:

$$
p_{e}^{m} x_{R}^{m} k_{R}^{m}+\left(1-p_{e}^{m}\right) x_{R}^{m} \bar{k}_{R}^{m}=x_{R}^{m} \forall m, k_{R}^{m}, \bar{k}_{R}^{m}
$$

That is, in expectation person $R$ earns exactly what is allocated to her in all distribution problems. In other words, a given allocation to $\mathrm{R}$ has the same expected value in all studied distribution problems. Finally, parameters are chosen in a way that distribution problem $m$ second order stochastically dominates distribution problem $m+1$, that is:

$p_{e}^{m} f\left(x_{R}^{m} k_{R}^{m}\right)+\left(1-p_{e}^{m}\right) f\left(x_{R}^{m} \bar{k}_{R}^{m}\right)>p_{e}^{m+1} f\left(x_{R}^{m+1} k_{R}^{m+1}\right)+\left(1-p_{e}^{m+1}\right) f\left(x_{R}^{m+1} \bar{k}_{R}^{m+1}\right)$

for every concave function $f(\cdot)$ (Mas-Colell et al., 1995 page 197). For convenience, we say that problem $m+1$ is riskier than problem $m$ if (3.3) is satisfied.

To summarize, we design our distribution problems in a way that ensures that in expectation the distribution of $X$ between person $\mathrm{R}$ and person 
$\mathrm{C}$ is always equal to the amount allocated to them, while the riskiness of person R's allocation changes across problems. This setup allows studying how the riskiness of a situation influences distributive justice views, ruling out the possibility that differences in allocations within and across distribution problems may be motivated by efficiency concerns.

The expressions below describe the expected values of the allocations $x_{R}^{m}, x_{C}^{m}$ in any of the $m$ distribution problems:

$$
E\left(x_{R}^{m}\right)=p_{e}^{m} x_{R}^{m} k_{R}^{m}+\left(1-p_{e}^{m}\right) x_{R}^{m} \bar{k}_{R}^{m}=x_{R}^{m}, E\left(x_{C}^{m}\right)=x_{C}^{m}
$$

In what follows we discuss the distributive justice principles that may motivate allocations in such distribution problems.

\subsubsection{Theoretical discussion}

Our analysis proceeds along the three principles that have inspired the development of most distributive justice theories (Konow, 2003).

For each principle, we discuss theories that are plausible candidates for motivating allocations. We then apply the discussed theories to the distribution problem that satisfies (3.4) in order to derive theoretically predicted allocations. These allocations, the so called fairness ideals (Cappelen et al., 2007), specify at the individual-level the fair amounts $x_{R}^{m}$ and $x_{C}^{m}$ that should be allocated to person $\mathrm{R}$ and person $\mathrm{C}$, respectively.

Equality Theories in this category typically reflect a concern for the well being of those who are least advantaged in society. Within this category, Egalitarianism (Deutsch, 1985) and Sen's Weak Equity Axiom (Sen, 1973) can be applied to make predictions in the context of our distribution problems. ${ }^{3}$ Egalitarianism prescribes equality of outcomes. Sen's weak equity axiom, in direct opposition to Utilitarianism (discussed below), prescribes to allocate more to the individual who derives less utility from a given quantity of the resource.

\footnotetext{
${ }^{3}$ Also the contributions of Rawls (1971) and Marx (1958) to distributive justice belong to this category. However, they do not seem applicable to the distribution problems we consider because no information on recipients' needs and initial wealth is available.
} 
Applying Egalitarianism to the distribution problems we study is not straightforward, because the state of nature is unknown at the moment of the division of $X$. Therefore, there is no allocation which guarantees that person $\mathrm{R}$ and $\mathrm{C}$ enjoy equal amounts after uncertainty is resolved. Given this impossibility, the allocation closest to the egalitarian ideal is the one that minimizes the expected difference between R's and C's final allocations. Formally stated:

$$
\min _{x_{R}^{m}, x_{C}^{m}} p_{e}^{m}\left|k_{R}^{m} x_{R}^{m}-x_{C}^{m}\right|+\left(1-p_{e}^{m}\right)\left|\bar{k}_{R}^{m} x_{R}^{m}-x_{C}^{m}\right| \text { s.t. } x_{R}^{m}+x_{C}^{m}=X
$$

The solution of the above minimization problem yields the following normatively fair allocations: ${ }^{4}$

$$
x_{R}^{m E g l}=\frac{X}{k_{R}^{m}+1}, x_{C}^{m E g l}=\frac{X k_{R}^{m}}{k_{R}^{m}+1}
$$

In all distribution problems $k_{R}^{m}>1$, therefore the result in (3.5) tells that the fair allocation to person $\mathrm{R}$, who bears the risk, is smaller than that to person $\mathrm{C}$, who does not bear any risk. Since such allocations aim at the smallest possible level of inequality after uncertainty is resolved, we call this fairness ideal ex-post equality.

The application of Sen's weak equity axiom to the discussed distribution problems requires knowledge of the recipients' utility function for money. For the sake of parsimony and in accordance with the literature (Wakker, 2010) we assume that both persons are characterized by the same, CRRA, utility function for money $U(x)=x^{\alpha}$. Assume now, for example, that $0<$ $\alpha<1$, which means that $\mathrm{R}$ and $\mathrm{C}$ are risk averse. This implies that in any of the $m$ distribution problems if the same amount is allocated to person $\mathrm{R}$ and person $\mathrm{C}$, this yields the former a lower expected utility than the latter. Hence, to satisfy Sen's axiom person $R$ should always be allocated more than person C. Put differently, person $R$ should be compensated because her disadvantaged position, namely facing uncertainty, is due to reasons beyond her personal influence.

Notice that Sen's weak equity axiom does not specify the extent to which

\footnotetext{
${ }^{4}$ The formal derivation of the normatively fair allocations can be found in the Appendix.
} 
person $\mathrm{R}$ should be compensated. In order to apply the axiom we assume that compensation is full, meaning that allocations are chosen in a way that $\mathrm{R}$ enjoys in expectation the same utility as $\mathrm{C}$ :

$$
E\left[U\left(x_{R}^{m}\right)\right]=E\left[U\left(x_{C}^{m}\right)\right] \text { s.t. } x_{R}^{m}+x_{C}^{m}=X
$$

The following equation expresses this idea in the context of the discussed distribution problems:

$$
p_{e}^{m}\left(x_{R}^{m} k_{R}^{m}\right)^{\alpha}+\left(1-p_{e}^{m}\right)\left(x_{R}^{m} \bar{k}_{R}^{m}\right)^{\alpha}=\left(x_{C}^{m}\right)^{\alpha}
$$

which can be rewritten to obtain the following fair allocations:

$$
x_{R}^{m} \operatorname{Sen}=\frac{X}{e^{\frac{Z}{\alpha}}+1}, x_{C}^{m} \operatorname{Sen}=\frac{X e^{\frac{Z}{\alpha}}}{e^{\frac{Z}{\alpha}}+1},
$$

where $Z=\ln \left[p_{e}^{m}\left(k_{R}^{m}\right)^{\alpha}+\left(1-p_{e}^{m}\right)\left(\bar{k}_{R}^{m}\right)^{\alpha}\right]$. From the expression in (3.2) it follows that $Z<0 \forall 0<\alpha<1$ and hence that $e^{\frac{Z}{\alpha}}<1$. Thus, the equations in (3.6) imply that in all distribution problems characterized by uncertainty person $\mathrm{R}$ should be allocated more than person $\mathrm{C}$. Further, $Z$ is decreasing in the riskiness of the distribution problem (see the inequality in (3.3)) implying that the fair allocation to $\mathrm{R}$ increases.

Note that the allocation in (3.6) is fair also when $\alpha>1$, that is when the recipients are characterized by risk seeking preferences. However, in such a case, $\mathrm{R}$ should be allocated less than $\mathrm{C}$ the riskier the division problem. Clearly, if preferences are risk neutral both recipients should be allocated the same amount.

As this fairness ideal guarantees that $\mathrm{R}$ and $\mathrm{C}$ enjoy the same utility in expectation we call it ex-ante equality.

Utilitarianism According to utilitarianism (Bentham, 1789 and Sen, 1979), one should pursue the greatest aggregate level of utility. This implies that more of a resource should be allocated to the person that derives the greater utility from it. As above, we assume that person $\mathrm{R}$ and person $\mathrm{C}$ are characterized by a power utility function for money. We also assume for the 
moment that preferences are risk averse. As a consequence in all distribution problems the same amount yields a lower expected utility to person $\mathrm{R}$ as compared to person C. Evidently, utilitarianism would then prescribe to allocate to person $\mathrm{R}$ a smaller share of $X$. The below maximization problems formalizes this intuition:

$$
\max _{x_{R}^{m}, x_{C}^{m}} E\left[U\left(x_{R}^{m}\right)+U\left(x_{C}^{m}\right)\right] \text { s.t. } x_{R}^{m}+x_{C}^{m}=X
$$

which for the discussed distribution problems becomes:

$$
\max _{x_{R}^{m}, x_{C}^{m}} p_{e}^{m}\left(x_{R}^{m} k_{R}^{m}\right)^{\alpha}+\left(1-p_{e}^{m}\right)\left(x_{R}^{m} \bar{k}_{R}^{m}\right)^{\alpha}+\left(x_{C}^{m}\right)^{\alpha} \text { s.t. } x_{R}^{m}+x_{C}^{m}=X
$$

The solution of the maximization problem leads to the following optimal allocations:

$$
x_{R}^{m U t i l}=\frac{X}{e^{-\frac{Z}{1-\alpha}}+1}, x_{C}^{m U t i l}=\frac{X e^{-\frac{Z}{1-\alpha}}}{e^{-\frac{Z}{1-\alpha}+1}}
$$

where $Z=\ln \left[p_{e}^{m}\left(k_{R}^{m}\right)^{\alpha}+\left(1-p_{e}^{m}\right)\left(\bar{k}_{R}^{m}\right)^{\alpha}\right]$. Since $e^{-\frac{Z}{1-\alpha}}>1 \forall 0<\alpha<1$ (see the previous section) the expressions in (3.7) imply that $R$ is allocated less than C. Further, the higher the riskiness of R's final earnings the less $R$ is allocated.

If $\alpha>1$, that is recipients are characterized by risk seeking preferences, the maximization of total welfare implies that the total amount of resources $X$ is allocated to $\mathrm{R}$. We call this fairness ideal utilitarian.

Equity This principle inspires theories that establish a dependence of normatively fair allocations on individual actions. The accountability principle (Konow, 1996) represents a generalization of Equity. In redistributing the output of a joint effort, allocations should be proportional to the inputs people control (like effort) but should not be related to those exogenous factors that people cannot influence but that nevertheless affect the output (like physical handicap or random events). The application of the accountability principle to our problems disregards the fact that the state of nature is uncertain at the moment of the division. Indeed, the total amount of resources 
to be divided solely depends on the productivity of person $\mathrm{R}$ and $\mathrm{C}$ which is assumed to be equal. It follows that the prescribed distribution is the equal split of $X$ in every distribution problem. Formally,

$$
x_{R}^{m E q}=x_{C}^{m E q}=\frac{X}{2}
$$

We call this fairness ideal equity. Lastly, notice that all discussed fairness ideals prescribe an equal split of the joint account when the final outcomes of both person $\mathrm{R}$ and $\mathrm{C}$ are independent of which event is realized and thus equal to their allocations. This can be easily seen by substituting $k_{R}^{m}=\bar{k}_{R}^{m}=1$ into the above equations.Table 4.3 summarizes the distributional implications of the four fairness ideals. ${ }^{5}$

Table 3.1: Fairness ideals.

\begin{tabular}{lccc}
\hline \hline & allocation to R & Relation of $x_{R}^{m}$ to $x_{C}^{m}$ & Relation of $x_{R}^{m}$ to riskiness \\
\hline ex-post equality & $x_{R}^{m}=\frac{X}{k_{R}^{m}+1}$ & $<$ & non-monotonic \\
ex-ante equality & $x_{R}^{m}=\frac{X}{e^{\frac{Z}{\alpha}}+1}$ & $>$ & increasing \\
utilitarian & $x_{R}^{m}=\frac{X}{e^{-\frac{Z}{1-\alpha}+1}}$ & $<$ & decreasing \\
equity & $x_{R}^{m}=\frac{X}{2}$ & $\equiv$ & constant \\
\hline
\end{tabular}

Note: The relations described above hold for $0<\alpha<1$.

\subsection{The Benevolent Dictator experiment}

The main purpose of this experiment is to investigate people's ideas about fairness in distribution problems where uncertainty affects the final distribution of rewards. The experiment consists of three parts. In the first part of the experiment subjects having the role of receivers work on a real effort task. The money earned by each subject is then deposited in a group account.

\footnotetext{
${ }^{5}$ Some of the relations in the second and third columns of the table only hold under the assumption that $0<\alpha<1$. More specifically, for $\alpha>1$ Sen's weak equity axiom prescribes that $x_{R}<x_{C}$ and that $x_{R}$ decreases with the riskiness of the situation. Further, if $\alpha>1$ Utilitarianism assigns the total amount of resources to $\mathrm{R}$ in all distribution problems.
} 
In the second part of the experiment a subject, the Benevolent Dictator, is asked to split the money in the account between the two recipients, himself having no stakes in the amount to divide (Konow, 2000). In the third and last part, subjects face a series of incentivized choices that allow estimating participants' preferences over uncertain prospects and beliefs about others' preferences. In what follows the experiment is described in detail.

Part 1: production After every participant takes a seat at the designated computer station, instructions for the first and second part of the experiment are distributed and read aloud by the experimenter. Participants are then randomly matched in groups of three; one group member is randomly assigned the role of $\mathrm{BD}$, the other two persons are the recipients, which we call $\mathrm{R}$ and $\mathrm{C}{ }^{6}$ In the first part of the experiment $\mathrm{R}$ and $\mathrm{C}$ subjects work individually on a real effort task (Gill and Prowse, 2009) while the BD is idle.

The real effort task consists of a single screen displaying 32 sliders on horizontal bars. Each slider can be moved at any point of the bar for an unlimited number of times and the actual position of a slider is displayed to the right of it. A slider is correctly positioned when the number 50 pops up at the right of the bar, that is when the slider is exactly at the middle of the bar. The subject's "score" in the task, interpreted as productivity, is the number of sliders positioned at 50 in 6 minutes time. The current score and the amount of time remaining are displayed on the top of the screen.

We chose the slider task because it is simple to communicate and to understand, it is identical across repetitions and it has no scope for guessing, so the number of correctly positioned sliders corresponds closely to the effort exerted by the subject. Effort in the slider task is incentivized: for each correctly positioned slider $€ 0.25$ are credited, so that subjects can credit up to $€ 8$ each. After the time for the task has expired, the BD, $\mathrm{R}$ and $\mathrm{C}$ subjects in the same group view the effort provided by $\mathrm{R}$ and $\mathrm{C}$. The total amount of money generated by such effort is deposited in a group account; members of the same group are then informed about the amount of money in their account. We chose the number of sliders on the screen and the available time

\footnotetext{
${ }^{6}$ In the experiment subjects are assigned the more neutral labels A, B and C.
} 
to complete the task in a way that maximum effort is easily achievable, as we intended that $R$ and $C$ subjects in the same group do not differ in terms of productivity and are thus equally deserving.

Part 2: allocation decisions In the second part of the experiment the $\mathrm{BD}$ has to divide the amount in the group account between $\mathrm{R}$ and $\mathrm{C}$, who are not active in this phase. The fact that $\mathrm{R}$ and $\mathrm{C}$ exerted effort and are thus not mere money recipients should increase BD's motivation to make a fair division. The $\mathrm{BD}$ does not have stakes in the division: her payment for this task is randomly and publicly determined at the end of the experiment and is not related in any way to the amount in the group account. More precisely, the BD can earn 4, 6, 10 or 12 Euro with equal chance, which means that she earns $€ 8$ in expectation. Given that $€ 8$ is probably the equal split of most group accounts, we avoided including 8 among the possible earnings as it may become a focal point for BD's allocation decisions.

The BD has to make a division in $m=7$ problems. We consider distribution problems characterized by risk ( $p_{e}^{m}$ is a known value) as well as by ambiguity ( $p_{e}^{m}$ is unknown). The latter form of uncertainty is more common in real life as people hardly know the objective probability of most events. On the other hand, experimental data about the former are easier to interpret and organize with the tools of microeconomic theory.

Table 3.2 shows the characteristics of each distribution problem. In the first distribution problem, Certainty, R's and C's final earnings are respectively equal to the amount $x_{R}^{1}$ and $x_{C}^{1}$ assigned to them by the BD. This is different in the division problems involving uncertainty. For instance, in division problem 4 -Risk, $p_{e}^{4}=0.5$ and $k_{R}^{4}=2, \bar{k}_{R}^{4}=0$. This means that $\mathrm{R}$ earns twice her allocation with $50 \%$ chance and earns nothing with $50 \%$ chance. In division problem 6-Ambiguity $p_{e}^{6}$ is unknown, $k_{R}^{6}=1.5, \bar{k}_{R}^{6}=0.5$. Differently stated, R's final earnings may be equal to 1.5 times or to 0.5 times her allocation, but likelihoods are unknown. Notice that for all division problems $m<6$, problem $m$ second order stochastically dominates problem $m+1 .^{7}$

\footnotetext{
${ }^{7}$ Distribution problems 6 and 7 are characterized by ambiguity and therefore cannot be ranked according to second order stochastic dominance.
} 
Table 3.2: Distribution problems.

\begin{tabular}{llc}
\hline \hline distribution problem & final earnings of R & final earnings of C \\
\hline 1-Certainty & $x_{R}^{1}$ & $x_{C}^{1}$ \\
2-Risk & $\left(0.5: x_{R}^{2} \times 1.5, x_{R}^{2} \times 0.5\right)$ & $x_{C}^{2}$ \\
3-Risk & $\left(0.8: x_{R}^{3} \times 1.25, x_{R}^{3} \times 0\right)$ & $x_{C}^{3}$ \\
4-Risk & $\left(0.5: x_{R}^{4} \times 2, x_{R}^{4} \times 0\right)$ & $x_{C}^{4}$ \\
5-Risk & $\left(0.2: x_{R}^{5} \times 5, x_{R}^{5} \times 0\right)$ & $x_{C}^{5}$ \\
6-Ambiguity & $\left(p: x_{R}^{6} \times 1.5, x_{R}^{6} \times 0.5\right)$ & $x_{C}^{6}$ \\
7-Ambiguity & $\left(p: x_{R}^{7} \times 2, x_{R}^{7} \times 0\right)$ & $x_{C}^{7}$ \\
\hline
\end{tabular}

Note: The numbers in parenthesis refer to a prospect as follows: $\left(\mathrm{p}_{e}^{m}: x_{R}^{m} \times k_{R}^{m}, x_{R}^{m} \times \bar{k}_{R}^{m}\right)$.

Distribution problems appear on the screen one at a time and the order of appearance is randomized at the group level.

At the end of the experiment only one problem is randomly selected to be relevant for the payment of $R$ and C. A stack of cards numbered form 1 to 100 is used to determine R's earnings in risky decision problems. For instance, if $\mathrm{R}$ faces a $50 \%$ chance of doubling her allocation, her allocation is eventually doubled only if a card with a number smaller than 51 is drawn. In order to operationalize ambiguity, a stack of 100 cards colored black and red is used. Neither the participants nor the experimenter knows the exact color composition of the stack, and each participant is free to choose his/her winning color at the beginning of the experiment. This procedure is described in the instructions that are distributed before the start of the experiment. The determination of earnings took place publicly at the end of the experiment so that subjects could witness how the chance devices were operated.

Part 3: Individual characteristics In the last part of the experiment we gather data on individual characteristics that may be related to BD's allocations decisions.

First of all, we measure subjects' risk preferences. Participants' certainty equivalents are elicited for 6 two outcomes lotteries. Table 4.2 shows the outcomes and the probabilities employed. For each lottery subjects see a 
screen on the computer that contains a description of the lottery and a list of 20 equally spaced sure amounts, ranging from the lottery's highest to lowest outcome. In order to facilitate comprehension, probabilities are expressed both in percentage points and with the aid of a pie chart. In each row of the decision screen subjects have to make a choice between the lottery and the sure amount. Subjects are not allowed to switch back and forth between the sure amount and the lottery. ${ }^{8}$ In other words, a unique switching point is elicited for each lottery. Certainty equivalents are then calculated as the arithmetic mean of the smallest sure amount preferred to the lottery and the consecutive sure amount on the list.

Table 3.3: Lotteries for risk preferences elicitation.

\begin{tabular}{cccc}
\hline \hline Lottery & $p$ & $y$ & $z$ \\
\hline 1 & 0.20 & 40 & 0 \\
2 & 0.50 & 16 & 0 \\
3 & 0.80 & 10 & 0 \\
4 & 0.50 & 12 & 4 \\
5 & 0.25 & 16 & 4 \\
6 & 0.33 & 12 & 0 \\
\hline
\end{tabular}

Note: The lottery outcome is $€ y$ with probability $p, € z$ with probability $1-p$.

We chose the above lotteries in order to measure risk preferences for the range of outcomes that are relevant in the distribution problems of the first part. In particular, note that the first 4 lotteries in the table all have an expected value of $€ 8$ and are characterized by the same probability values that appear in the risky distribution problems. Lottery 5 and 6 provide additional information on risk preferences in the range of outcomes relevant to our experiment.

As two distribution problems are characterized by ambiguity, we also

\footnotetext{
${ }^{8}$ Consistency is enforced as it is crucial for the elicitation of beliefs about others' preferences.
} 
elicit subjects' attitude to it. To this end, subjects face 6 decision screens where they make choices between an ambiguous lottery and several risky ones. Both the ambiguous and the risky lotteries in a given decision screen are characterized by the same outcomes pair, which are those in table 3.3. In each screen subjects see a description of the ambiguous lottery and a list of 20 risky lotteries. The first and the last risky lotteries on the list are both degenerate, and guarantee respectively the high and the low outcome of the lottery. In every row of the list the likelihood of the high outcome decreases by $5 \%$, while the likelihood of the low outcome increases by $5 \%$. In each decision screen, subjects can switch only once from the risky to the ambiguous lottery. As in the first part of the experiment, ambiguity is generated with a deck of red and black cards in unknown color composition. Thus, the switching point provides an interval for a subject's belief about the likelihood that a winning card is drawn.

At the end of the experiment one out of the 12 decision screens (ambiguous and risky) and one row within the selected decision screen are randomly selected for payment. The relevant lottery is then publicly played out and earnings are added to those of the first part.

Lastly, we investigate subjects' beliefs about others' risk and ambiguity preferences. In order to do so, we ask subjects to estimate the choices made by a randomly matched group member in 4 risky and 2 ambiguous decision screens. Rather than eliciting a point belief, we decided to elicit an interval: for a certain decision screen, each participant is asked to indicate what he/she thinks is the minimum and the maximum switching point of the matched group member. The belief elicitation is incentivized using the interval scoring rule (Schlag and Van der Weele, 2009). The rule works as follows: if the true switching point of the matched member lies within the minimum and maximum of the indicated interval, the subject earns an amount that is inversely related to the length of the indicated interval, which could also be a point. If the true switching point of the matched member lays outside the indicated interval, the subject earns nothing.

The interval scoring rule has two advantages compared to scoring rules that elicit point beliefs. First, it is less time consuming and cognitively demanding for the subjects because to know the support of the beliefs' distribution the experimenter does not need to elicit probabilities over all possible events. 
Second, the interval scoring rule allows inferences that are valid under any degree of subjects' risk aversion and not only when subjects are risk neutral (Schlag and Van der Weele, 2009).

Before being privately payed out in cash and dismissed, subjects are asked a few socioeconomic questions. Furthermore, they are asked to complete a debriefing questionnaire on the first part of the experiment. More precisely, for each distribution problem subjects in the same group are reminded of the allocations made by the $\mathrm{BD}$. The $\mathrm{BD}$ is then asked to explain how he/she made the allocation decisions, while $\mathrm{R}$ and $\mathrm{C}$ subjects are asked if they deem the division fair or unfair, and why.

90 students from Maastricht University participated in the computerized experiment which was conducted in January 2010 in the Behavioral and Experimental Lab (BEElab) at the Maastricht University School of Business and Economics, using the Z-Tree software (Fischbacher, 2007). $82 \%$ of the subjects were enrolled in the School of Business and Economics and the remaining $18 \%$ were enrolled in other faculties, such as law, medicine and arts. $47 \%$ of the subjects were male. The average age was 23.5 years. The experiment lasted approximately 80 minutes and the average earnings per subject were $€ 17$.

\subsection{Results}

We begin with presenting descriptive statistics on production, allocation decisions and individual characteristics.

Production and allocation decisions Our design of the production phase successfully induced maximum performance, which was achieved by both $\mathrm{R}$ and $\mathrm{C}$ in 28 out of 30 groups. In the following analysis we exclude the 2 groups where performance was not maximal by one, or both, group members because we want to rule out any effects such differences may have on the BD's allocation decisions. Consequently, for all analyzed groups the group account equals $€ 16$.

Consider now the first distribution problem which is characterized by the fact that both R's and C's final earnings are equal to the amount allo- 
cated to them. As shown in Section 3.2 this is the only problem where all the applicable theories of justice prescribe an equal split of the group account. Therefore, allocations in this problem provide a test of whether our approach is successful in eliciting people's fairness ideals. The histogram in Figure 3.1 clearly shows that, with the exception of only two outliers, Benevolent Dictators indeed split the amount in the group account equally between $\mathrm{R}$ and $\mathrm{C}$. This result demonstrates that equally deserving individuals are treated equally when the earnings' distribution does not depend on the state of nature.

Figure 3.1: Distribution problem 1-Certainty.

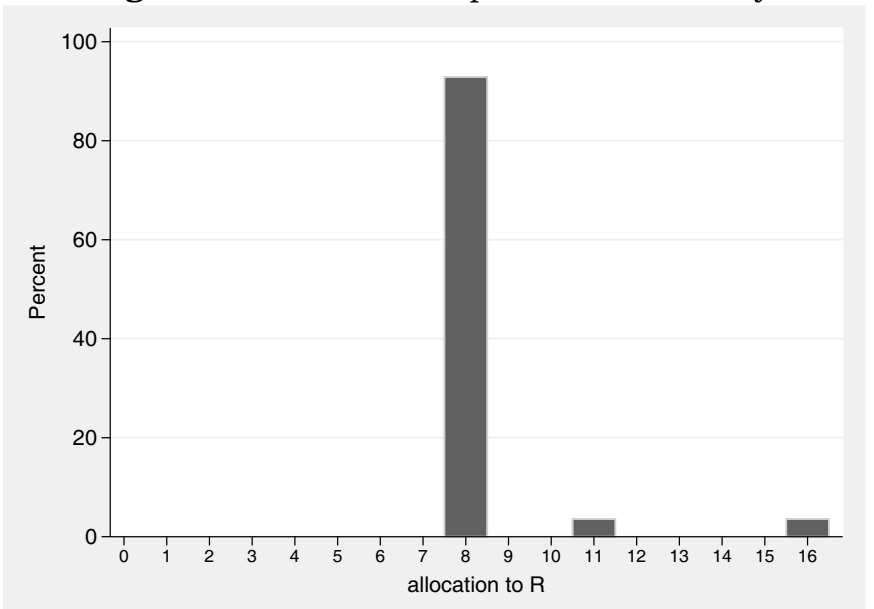

Allocation behavior changes dramatically in distribution problems characterized by uncertainty. The histograms in Figure 3.2 depict allocations to $\mathrm{R}$ in these six distribution problems.

They clearly show that within each distribution problem allocations are highly heterogeneous and that allocations substantially differ also across the distribution problems.

Table 3.4 provides descriptive statistics and statistical tests on allocations to $\mathrm{R}$ subjects; all statistical tests employed in the analysis are twosided. In the last but one column of the table we report the results of a 
Figure 3.2: Distribution problems characterized by uncertainty.

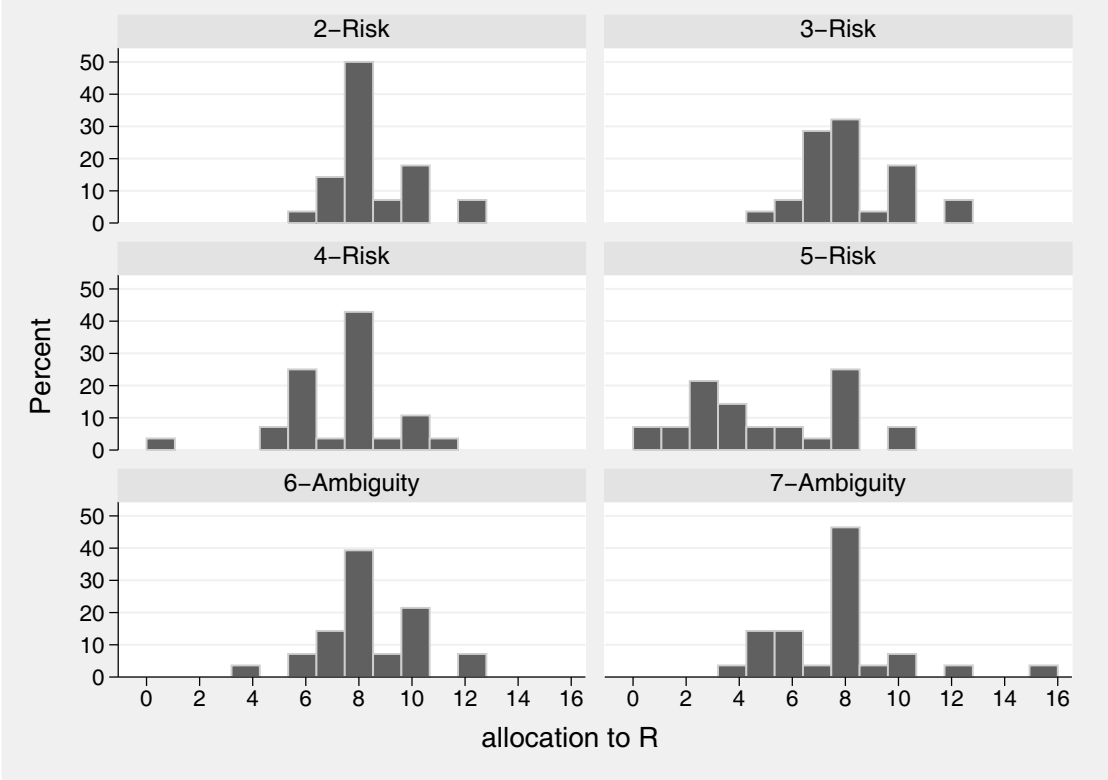

Wilcoxon test, which is used to test whether allocations to $R$ are significantly different from the equal split of $€ 8$. We find that the median allocation to $\mathrm{R}$ is significantly smaller than the equal split only in distribution problem 5-Risk. ${ }^{9}$ However, given that the median allocation to $\mathrm{R}$ is almost always $€ 8$, results of the Wilcoxon test only provide limited information about the existing heterogeneity within distribution problems. Therefore, for each risky problem we also compare the distribution of allocations to the hypothetical distribution where an equal split is uniformly chosen, using the Kolmogorov-Smirnov test (henceforth K-S test). We find that in all risky distribution problems the distribution of allocations is significantly different from an equal-split distribution ( $p$-value at least 0.07 ).

The results for the two distribution problems characterized by ambiguity are similar. The median allocation to $\mathrm{B}$ is not significantly different from the equal split in both distribution problems. However standard deviations

\footnotetext{
${ }^{9}$ When using a one-sample t-test we find that the mean allocation to $\mathrm{R}$ is significantly smaller than 8 in 4-Risk and significantly higher than 8 in 2-Risk.
} 
are quite high and a K-S test confirms that distributions significantly differ from the equal-split distribution.

Table 3.4: Allocations to $\mathrm{R}$ in all distribution problems.

\begin{tabular}{|c|c|c|c|c|c|c|c|}
\hline \multirow[b]{2}{*}{ distribution problem } & \multicolumn{6}{|c|}{ allocation to $\mathrm{R}$} & \multirow[b]{2}{*}{ K-S test } \\
\hline & $\max$ & $\min$ & mean & median & s. deviation & Wilcoxon test & \\
\hline \multirow[t]{2}{*}{ 1-Certainty } & 16 & 8 & 8.39 & 8 & 1.59 & $\mathrm{z}=1.41$ & $\mathrm{p}=1.00$ \\
\hline & & & & & & $(p=0.16)$ & \\
\hline \multirow[t]{2}{*}{ 2-Risk } & 12 & 6 & 8.50 & 8 & 1.43 & $\mathrm{z}=1.40$ & $\mathrm{p}=0.07$ \\
\hline & & & & & & $(p=0.16)$ & \\
\hline \multirow[t]{2}{*}{ 3-Risk } & 12 & 5 & 8.13 & 8 & 1.69 & $\mathrm{z}=-0.13$ & $\mathrm{p}=0.006$ \\
\hline & & & & & & $(\mathrm{p}=0.90)$ & \\
\hline \multirow[t]{2}{*}{ 4-Risk } & 11 & 0 & 7.33 & 8 & 2.10 & $\mathrm{z}=-1.57$ & $\mathrm{p}=0.015$ \\
\hline & & & & & & $(\mathrm{p}=0.12)$ & \\
\hline \multirow[t]{2}{*}{ 5-Risk } & 10 & 0 & 5.10 & 4.50 & 2.84 & $z=-3.86$ & $\mathrm{p}=0.00$ \\
\hline & & & & & & $(\mathrm{p}=0.0001)$ & \\
\hline \multirow[t]{2}{*}{ 6-Ambiguity } & 12 & 4 & 8.36 & 8 & 1.75 & $\mathrm{z}=0.99$ & $\mathrm{p}=0.03$ \\
\hline & & & & & & $(p=0.32)$ & \\
\hline \multirow[t]{2}{*}{ 7-Ambiguity } & 16 & 4 & 7.73 & 8 & 2.39 & $\mathrm{z}=-1.24$ & $\mathrm{p}=0.03$ \\
\hline & & & & & & $(\mathrm{p}=0.21)$ & \\
\hline
\end{tabular}

Note: The null hypothesis of the Wilcoxon test is that the median allocation to $\mathrm{R}$ is equal to 8 . The null hypothesis in the K-S test is that the distribution of allocations to $\mathrm{R}$ is equal to the distribution where the equal split has density 1.

Result 3.4.1. The amount in the joint account is almost always split equally in the distribution problem where uncertainty is absent. In all distribution problems characterized by uncertainty the distribution of allocations is significantly different from the equal split.

As pointed out earlier, the histograms in Figure 3.2 show that Benevolent Dictators differentiated their allocations decisions according to the characteristics of the distribution problem. We now proceed by comparing all distribution problems pair-wise. Table 3.5 summarizes the differences between decision situations, where we correct for multiple comparisons us- 
ing the "false discovery rate" method (Benjamini and Hochberg, 1995). The most striking fact emerging from Table 3.5 is that allocations in 5-Risk are highly and significantly different from allocations in all other distribution problems. Furthermore, when ignoring the distribution problem characterized by the lowest level of riskiness, 2-Risk, significant differences are observed between allocations in 1-Certainty and allocations in problems characterized by risk. Interestingly, we do not observe significant differences between 2-Risk and 6-Ambiguity and 4-Risk and 7-Ambiguity respectively. Given that these pairs of prospects are characterized by the same potential outcomes this result indicates that Benevolent Dictators treat ambiguity no differently than a 50-50 prospect.

Table 3.5: Pair-wise comparisons of distribution problems.

\begin{tabular}{|c|c|c|c|c|c|c|}
\hline & 2-Risk & 3-Risk & 4-Risk & 5-Risk & 6-Ambiguity & 7-Ambiguity \\
\hline \multirow[t]{6}{*}{ 1-Certainty } & $x[x]$ & $\mathrm{x}[* * *]$ & $\mathrm{x}[* * *]$ & $* * *[* * *]$ & $\mathrm{x}[\mathrm{x}]$ & $\mathrm{x}[\mathrm{x}]$ \\
\hline & \multirow[t]{5}{*}{ 2-Risk } & $\mathrm{x}[\mathrm{x}]$ & $\mathrm{x}[\mathrm{x}]$ & $* * *[* * *]$ & $\mathrm{x}[\mathrm{x}]$ & $\mathrm{x}[\mathrm{x}]$ \\
\hline & & \multirow[t]{4}{*}{ 3-Risk } & $\mathrm{x}[\mathrm{x}]$ & $* * *[* * *]$ & $x[x]$ & $x[x]$ \\
\hline & & & \multirow[t]{3}{*}{ 4-Risk } & $* * *[* * *]$ & $\mathrm{x}[\mathrm{x}]$ & $\mathrm{x}[\mathrm{x}]$ \\
\hline & & & & \multirow[t]{2}{*}{ 5-Risk } & $* * *[* * *]$ & $* * *[* * *]$ \\
\hline & & & & & 6-Ambiguity & $x[x]$ \\
\hline
\end{tabular}

Note: Wilcoxon test and K-S test (the latter in brackets). An $\mathrm{x}$ indicates that there are no statistically significant differences. $* * *$ indicates significance at the $1 \%$ level, ** at the 5\% level. All results are corrected using the "false discovery rate" method.

The statistics in Table 3.4 also show that although a given allocation $x_{R}^{m}$ has the same expected value across all distribution problems, the average allocation to $R$ is negatively related to the distribution problems when ranked according to second order stochastic dominance. Furthermore, the standard deviation of allocations to $\mathrm{R}$ is negatively related to such ranking, suggesting that ideas about fair distributions are more dispersed in division problems with a high level of riskiness. For each BD we calculate the Spearman correlation coefficient between the allocations to $R$ and the rank of the risky problems according to second order stochastic dominance. We find that the average Spearman correlation coefficient is -0.42 and is negative for 18 
of the 28 Benevolent Dictators. ${ }^{10}$ A Wilcoxon test rejects the hypothesis that the correlation coefficient is equal to $0(p=0.001)$.

Result 3.4.2. Across distribution problems statistically significant differences in allocations' medians and distributions are observed. In particular, $R$ is allocated on average significantly less the higher the riskiness of her final earnings. No significant difference is found between risky and ambiguous distribution problems which share the same possible outcomes for a given allocation.

To summarize, our results so far show that allocations are highly heterogeneous within and across distribution problems. Furthermore, in the aggregate Benevolent Dictators significantly decrease their allocation to $\mathrm{R}$ with increasing riskiness of the distribution problem. In the following we zoom into the individual data and explore the relation between BD's own preferences, beliefs about other's preferences, and allocations.

Individual characteristics In the third part of the experiment participants make several decisions that allow estimating their attitude to risk and ambiguity.

First, we calculate subjects' certainty equivalent for each of the employed risky lotteries and assume a CRRA utility function for money $U(x)=x^{\alpha}$, where $0<\alpha<1$ indicates risk aversion, $\alpha=1$ indicates risk neutrality and $\alpha>1$ risk seeking preferences. Under these parametric assumptions, we estimate the parameter value of $\alpha$ for each subject by minimizing the sum of squared distances (Wakker, 2008 and 2010). That is;

$$
\min _{\alpha} \sum_{i=1}^{6}\left[\left(p_{i} y_{i}^{\alpha}+\left(1-p_{i}\right) z_{i}^{\alpha}\right)^{\frac{1}{\alpha}}-c e_{i}\right]^{2}
$$

where the first term in brackets indicates the theoretically predicted certainty equivalent for lottery $i$ and $c e_{i}$ is the elicited certainty equivalent of lottery $i$. To correct for heteroscedasticity prospects are normalized to uniform length.

We find that the average $\mathrm{BD}$ is characterized by $\alpha=0.77$, ( $s . d=0.24$, median $\alpha=0.79$ ), which is in accordance with earlier findings (see, for instance,

\footnotetext{
${ }^{10}$ The Pearson correlation coefficient is -0.44 .
} 
Holt and Laury, 2002). ${ }^{11}$

Second, we elicit subjects' ambiguity attitude by using their choices between ambiguous and risky lotteries. Assuming Subjective Expected Utility theory (Savage, 1954), such choices reveal the bounds of the probability interval containing a subject's prior belief on the ambiguous event. ${ }^{12}$ As the ambiguous event is the same in all the decision screens, subjects should consistently reveal the same prior belief in all decisions. When pair-wise comparing the elicited prior beliefs in all six decision screens we find that subjects indeed hold consistent beliefs (the p-value of the Wilcoxon signedrank test is at least 0.22). For each subject we construct a variable called "prior-belief" defined as the average of the prior beliefs elicited in the six decision screens. The mean prior-belief of Benevolent Dictators is equal to 0.46 (s.d. $=0.08$, median prior belief $=0.48$ ). Hence, the average BD believes that the probability of the ambiguous event is very close to $50 \%$, a probability value which indicates only a slight aversion to ambiguity. ${ }^{13}$ Notice that the standard deviation of prior-belief is very small, indicating a large degree of consistency among Benevolent Dictators.

Before the end of the experiment subjects are asked to provide their best estimate of the choices made by a randomly matched group member in 4 risky and 2 ambiguous decision screens. We use their estimates to construct a synthetic measure of their beliefs about others' risk and ambiguity preferences. First, taking the midpoint of the interval indicated by a subject, we calculate the beliefs about others' certainty equivalents for risky lotteries and others' prior beliefs on the ambiguous event. Using these measures we employ the same method as for the estimation of Benevolent Dictators' risk preferences to estimate what Benevolent Dictators believe to be the coefficient of relative risk aversion, $\alpha$, of other subjects. We use the average of the two elicited believed prior beliefs to get a measure of Benevolent Dictators'

\footnotetext{
${ }^{11}$ Pair-wise comparisons of BD's, R's and C's risk attitude reveal no statistically significant difference ( $p \geq 0.14$, Mann-Whitney test).

${ }^{12}$ We take the midpoint of the interval to be the subjects' prior belief. As the length of the interval is always equal to 0.05 taking the midpoint cannot result in a large bias.

${ }^{13}$ Pair-wise comparisons of BD's, R's and C's prior beliefs reveal no statistically significant difference, $p \geq 0.69$ Mann-Whitney test.
} 
belief about others' ambiguity attitude, where ambiguity is disliked more the smaller the prior belief on the ambiguous event.

We find that Benevolent Dictators' beliefs about others' preferences are highly, though imperfectly, correlated with their own risk and ambiguity preferences (Spearman's rho $=0.61, p=0.0006$ for risk preferences, Spearman's rho $=0.50, p=0.009$ for ambiguity preferences, Pearson correlation coefficients are 0.48 for risk preferences and 0.61 for ambiguity preferences). ${ }^{14}$ The estimation of preferences and beliefs allows investigating whether there exists a relation between these measures and allocation decisions. To test this we regress allocations to $\mathrm{R}$ in distribution problems characterized by uncertainty on BD's risk preferences, beliefs about others' risk preferences, prior beliefs on the ambiguous event and beliefs about others' attitude to ambiguity (the last two variables are interacted with a variable that takes the value 1 only in decision situation 6 and 7). We also include dummy variables for gender (the variable takes the value 0 for males and 1 for females) and for the ability to calculate expected values (the variable takes the value 1 if the subject could correctly calculate the expected value of a simple lottery; $54 \%$ of Benevolent Dictators answered correctly). ${ }^{15}$

Table 3.6 shows the regression results. We find that beliefs about others' risk preferences, prior beliefs on the ambiguous event, beliefs about others' priors, gender and the ability to calculate expected values are not significantly related to allocation decisions. However, allocations to $\mathrm{R}$ are negatively and marginally significantly related to BD's own measure of risk aversion $\alpha$. Hence the more risk averse a BD the more he/she gives to $\mathrm{R}$ in risky distribution problems. We note that the coefficient associated to BD's risk attitude remains significant when the variable describing beliefs about others' risk preferences is excluded from the regression. Further, when the own risk preference variable is excluded and the beliefs variable is included, the

\footnotetext{
${ }^{14}$ Two outliers believing that others' priors on the ambiguous event were below 0.12 , and thus extreme, are excluded when calculating correlations for ambiguity preferences. When they are included both the Pearson correlation coefficient and the Spearman's rho are equal to $0.27, \mathrm{p}=0.16$.

${ }^{15}$ The following, non-incentivized, question was administered at the end of the experiment: "We would like to ask you to calculate the expected value of the following lottery: with 50\% chance you earn 20 Euro and with 50\% you earn 10 Euro. Please type your answer in the box below."
} 
latter remains insignificant. We also note that, since own risk preferences and beliefs about others' risk preferences are highly correlated, regression coefficients are only informative regarding the direction and significance, but not the magnitude, of these relations.

Table 3.6: Allocations to $R$ in uncertain distribution problems, OLS regression.

\begin{tabular}{lccc}
\hline \hline & \multicolumn{3}{c}{ dependent variable: allocations to R } \\
\cline { 2 - 4 } independent variables & coefficient & standard error & p-value \\
\hline$\alpha$ & -2.21 & 1.21 & 0.08 \\
believed $\alpha$ & 0.84 & 0.86 & 0.34 \\
prior beliefs & 0.53 & 2.23 & 0.81 \\
believed prior beliefs & -0.84 & 1.56 & 0.6 \\
female & 0.19 & 0.35 & 0.58 \\
correct EV & 0.59 & 0.38 & 0.13 \\
constant & 9.81 & 1.28 & 0.00 \\
R-squared & 0.3 & & \\
Prob>F & 0.001 & & \\
$\mathrm{~N}$ & 156 & & \\
\hline
\end{tabular}

Note: Distribution problem dummies included and jointly significant. Standard errors are robust to heteroskedasticity and are clustered on subjects. Two subjects that acted randomly are excluded.

Result 3.4.3. BD's allocations to $R$ in risky distribution problems are positively and significantly related only to BD's own risk aversion.

To summarize, data from the third part of the experiment reveal the existence of a significant and positive relation between BD's risk aversion and her allocations to $\mathrm{R}$ in distribution problems characterized by risk.

\subsubsection{Fairness ideals}

In Section 2 we discussed theories of distributive justice in relation to the investigated distribution problems and analytically derived four fairness ideals. Here we use the derived expressions to compute the four fairness ideals 
at the individual level for each uncertain distribution problem. Finally, we estimate the distribution of these fairness ideals in our sample.

Consider the two fairness ideals inspired by the principle of Equality. First, the ex-post equality ideal specifies allocations that minimize the expected inequality between R's and C's final earnings. Second, the ex-ante equality ideal, based on Sen's weak equity axiom, prescribes that more money is allocated to the person that derives in expectation less utility from a given allocation. The expressions for the ex-ante fairness ideal are derived from the equalization of R's and C's expected utilities under the assumption that these are known. Since Benevolent Dictators in the experiment do not have information on recipients' preferences over uncertainty, we need to make assumptions on the value of $\alpha$ in equation (3.6). We assume that Benevolent Dictators evaluate the nature of R's position from their own viewpoint. Alternatively we could assume that Benevolent Dictators use their beliefs about others' preferences over uncertainty to compute the fair values of $x_{R}^{m}$ and $x_{C}^{m}$. However, our assumption seems reasonable as we observe that BD's own risk preferences and beliefs about others' risk preferences are not significantly different. Further, we also find that only BD's own risk preferences explain allocation decisions (see Result 3). Therefore, we proceed by substituting Benevolent Dictators' own $\alpha$ in the expression of the ex-ante equality ideal.

The third fairness ideal is the utilitarian one, which specifies allocations that maximize total welfare. This fairness ideal is subject to the same issues regarding preferences over uncertainty discussed above. We again proceed by assuming that the BD is acting in accordance with her own preferences. Fourth, the equity fairness ideal selects allocations that are proportional to the effort exerted in the creation of a joint output. As we only consider groups where productivity was highest by both $\mathrm{R}$ and $\mathrm{C}$, this fairness ideal prescribes an equal split in all distribution problems. Finally, in order to compute fairness ideals for ambiguous distribution problems we assume that $p_{e}^{6}=p_{e}^{7}=0.5$. This assumption is justified by invoking the principle of insufficient reason (Keynes, 1921), which states that if the possible states of the world are indistinguishable by the decision maker, equal probability should be assigned to each of them. BD's allocations in ambiguous 
distribution problems suggest that such an assumption is also empirically supported. As pointed out earlier (Result 4) allocations are indeed not related to BD's elicited prior-beliefs on the ambiguous event. Further, the mean and median prior-belief are both very close to 0.5 (see the paragraph on Individual characteristics). Table 3.7 shows the predicted allocations for all four fairness ideals calculated for the average coefficient of risk aversion $\alpha=0.77$.

Table 3.7: Prescribed allocations to $\mathrm{R}, \alpha=0.77$.

\begin{tabular}{lcccc}
\hline \hline & \multicolumn{4}{c}{ allocation to R under different fairness ideals } \\
\cline { 2 - 5 } distribution problem & ex-post & ex-ante & utilitarian & equity \\
\hline 1-Certainty & 8 & 8 & 8 & 8 \\
2-Risk & 6.40 & 8.10 & 7.60 & 8 \\
3-Risk & 7.10 & 8.30 & 7.10 & 8 \\
4-Risk & 5.30 & 8.90 & 5.30 & 8 \\
5-Risk & 2.70 & 10 & 2.70 & 8 \\
6-Ambiguity & 6.40 & 8.10 & 7.60 & 8 \\
7-Ambiguity & 5.30 & 8.90 & 5.30 & 8 \\
\hline
\end{tabular}

The second column of the Table shows that the ex-post ideal requires to allocate less money to $\mathrm{R}$ than to $\mathrm{C}$ in all distribution problems; however, the prescribed allocation to $\mathrm{R}$ is not monotonically related to the riskiness of the distribution problem. Instead, the ex-ante ideal establishes an increasing relation between R's compensation and the situations' riskiness; the values in column 3 would be even more distant from each other for higher values of $\alpha$. Column 4 shows that the utilitarian ideal prescribes monotonically decreasing allocations to R. Note that for distribution problems 3 to 5 utilitarian fairness ideals are equal to ex-post ideals: this is due to the fact that the experiment parameters are chosen to keep the expected value of an allocation constant across distribution problems and equal between $\mathrm{R}$ and $\mathrm{C}$, as 
explained in section 3.2. ${ }^{16}$

This feature is fundamental for the comparability of distribution problems and to interpret allocations decisions within a specific problem. Indeed, if expected values were not constant, allocations could be motivated by efficiency reasons, which we would not be able to separate from distributive justice considerations in the data.

After computing all the four fairness ideals at the individual level, we estimate the proportion of allocations consistent with each ideal. To this end, we assume that:

$$
y_{i}^{m}=x_{i}^{j m}+\epsilon_{i}^{m} \epsilon_{i}^{m} \sim N(0,1)
$$

Where $y_{i}^{m}$ is the amount allocated by BD $i$ to $\mathrm{R}$ in problem $m$ and $x_{i}^{j m}$ is the amount $\mathrm{R}$ should receive in problem $m$ according to fairness ideal $j$. Under these assumptions, for each $i$ and for $j=E g l$, Sen, Util, $E q$ we calculate the density of $y_{i}^{m}$ assuming a normal distribution with mean $x_{i}^{j m}$. We then proceed by rescaling the density values at the individual level to obtain a relative weighted ranking of the ideals. The associated weights are larger the closets the ideal to the observed allocation and the sum of all four weights is always equal to 100 . All weights are then averaged at the fairness ideal level: the obtained values can be interpreted as the relative weight, or importance, of each fairness ideal at the population level. We choose this procedure because the alternative minimization of squared residuals neglects the relative distance of ideals to allocations and only allows identifying the best fitting ideal for each allocation decision. In any case, we find very similar results when the fairness ideals are fitted to data by minimizing squared residuals: results are reported later on in the paper. Distribution problem 1-Certainty is not included in the estimation of the distribution of fairness ideals because all four ideals prescribe an equal split of

$$
\begin{aligned}
& { }^{16} \text { When } 0<\alpha<1 \text {, we have that } x_{R}^{E} g l=x_{R}^{U t i l} \text { because: } \\
& \frac{X}{k_{R}+1}=\frac{X}{e^{-\frac{Z}{1-\alpha}}+1} \Leftrightarrow k_{R}=e^{-\frac{Z}{1-\alpha}} \Leftrightarrow \ln k_{R}=\ln e^{\frac{\ln p_{e} k_{R}^{\alpha}}{\alpha-1}} \Leftrightarrow \\
& (\alpha-1) \ln k_{R}=\ln p_{e}+\ln k_{R}^{\alpha} \Leftrightarrow(\alpha-1) \ln k_{R}=\ln 1 / k_{R}+\alpha \ln k_{R}
\end{aligned}
$$


the group account in this problem.

Result 3.4.4. In distribution problems characterized by uncertainty, $21 \%$ of the allocations are consistent with ex-post equality, 29\% with ex-ante equality, $17 \%$ with utilitarian fairness and $33 \%$ with equity.

This result shows that each of the four fairness ideals motivates a significant share of allocations. ${ }^{17} 1819$ Though one-third of the allocations is motivated by the equity ideal, which neglects effect of uncertainty on final earnings, the majority of allocations is consistent with fairness ideals that account for the presence of uncertainty. In order to understand how well the theoretically specified ideals fit our data, we calculate for each allocation the error $\epsilon_{i}^{m}$ associated to the best fitting fairness ideal.

We find that the mean error is very close to 0 , namely $€-0.32$, the median error is 0 and $74 \%$ of allocations differ from the best fitting ideal by at most $€ 1$. Hence, we are confident that a large majority of the observed allocations is very well represented by at least one of the four ideals. ${ }^{20}$ We also find support for the identified fairness ideals in the debriefing questionnaire administered at the end of the experiment. In the appendix we report some answers Benevolent Dictators gave to the question of how they made their allocation decisions.

Since mean allocations and allocations' distributions are related to the problems' riskiness, it is an interesting question whether the endorsement of

\footnotetext{
${ }^{17}$ When minimizing the sum of squared residuals $23 \%$ of the allocations are consistent with ex-post equality, $25 \%$ with ex-ante equality, $12 \%$ with utilitarian fairness and $40 \%$ with equity.

${ }^{18}$ Results change only slightly if we exclude distribution problems characterized by ambiguity: $22 \%$ of the allocations are consistent with ex-post equality, $28 \%$ with ex-ante equality, $18 \%$ with utilitarian fairness and $32 \%$ with equity.

${ }^{19}$ The estimated shares only change by few percentage points if different assumptions on the standard deviation of $\epsilon_{i}^{m}$ are introduced (for instance, s.d.=2). Importantly, the relative ranking of fairness ideals is unchanged.

${ }^{20} \mathrm{We}$ also estimate the best fitting ideal at the subject level, that is the ideal that minimizes the sum of squared residuals over the 6 distribution problems characterized by uncertainty. We find that the allocation choices of $15.4 \%$ of the subjects are best represented by the exante ideal, $15.4 \%$ by the ex-post ideal, $26.9 \%$ by the utilitarian ideal and the remaining $42.3 \%$ by equity. Two subjects who acted randomly are excluded from the estimation. The mean residual is €2.9.
} 
fairness ideals is also related to the riskiness of the distribution problems. Figure3.3 shows the relative weight of each fairness ideal at the distribution problem level. Clearly, the weigh of the ex-post and the ex-ante ideals changes in a quasi-monotonic and opposite way over the distribution problems.

Figure 3.3: Relative weight of fairness ideals by distribution problem.

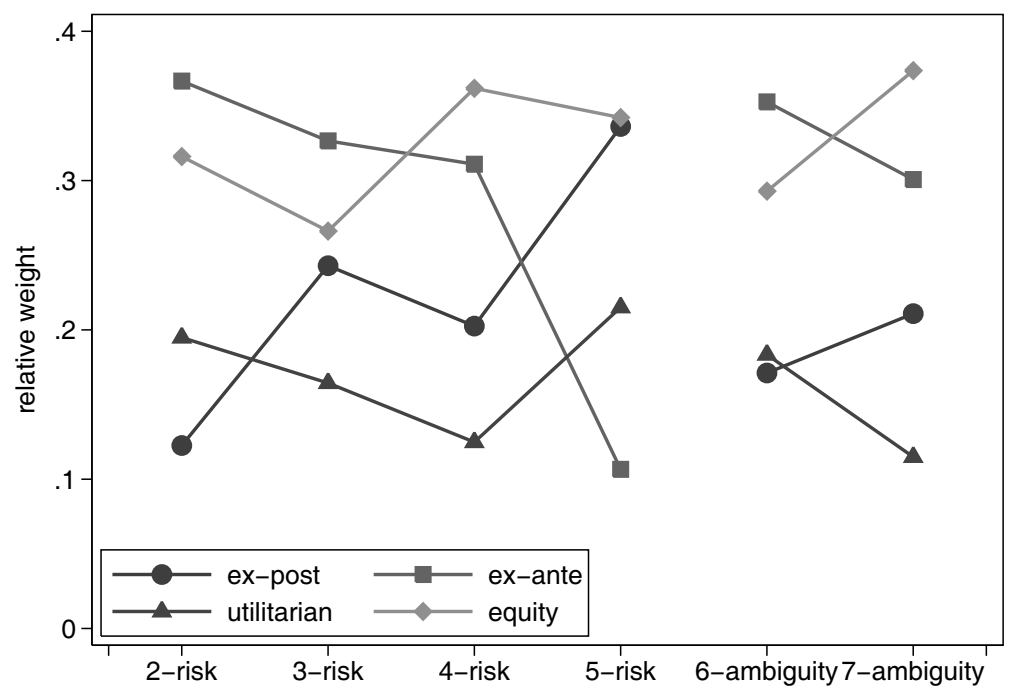

In particular, when uncertainty is highest, in 5-Risk, on average the equity ideal together with the ex-post fairness is characterized by the largest weight (both 34\%). Conversely, when uncertainty is relatively low, as in 2Risk, the ex-ante ideal gets the largest weight (37\%).

Using the Spearman correlation coefficient, for each BD we calculate whether the weights of a given fairness ideal in the different distribution problems are correlated to the riskiness of the distribution problems. We find that Benevolent Dictators' endorsement of ex-post fairness is positively, but insignificantly, correlated with the riskiness of the distribution problem (the mean correlation coefficient is 0.13 , a Wilcoxon test does not allow to reject the hypothesis that the correlation coefficient is equal to $0, \mathrm{p}=0.2$ ) while the endorsement of ex-ante fairness is negatively and significantly related to 
the riskiness of the distribution problem (the mean correlation coefficient is -0.43 , Wilcoxon test $\mathrm{p}=0.01$ ). Correlation coefficients are negatively but not significantly different than 0 for the utilitarian and the equity ideal (correlation utilitarian ideal -0.18 , Wilcoxon test $\mathrm{p}=0.16$, correlation equity ideal -0.15 Wilcoxon test $\mathrm{p}=0.21) .{ }^{21}$

Result 3.4.5. Subjects' endorsement of ex-post equality is positively, although not significantly, related to the riskiness of the distribution problem, while support for ex-ante equality significantly decreases with it. Support for the utilitarian and the equity ideal are not significantly related to the riskiness of the distribution problem.

This implies that the principle of Equality, which is the inspiration for both the ex-post and the ex-ante equality fairness ideals, is interpreted differently depending on the riskiness of the distribution problem. In particular, when subjects' final positions are moderately risky the largest weight is on the compensation of the disadvantaged, while concerns for ex-post fairness weigh more when risk can potentially lead to very unequal distributions. This finding support the idea that contextual elements influence justice ideas (Konow, 2003). Theories of justice are based on general principles but their actual application requires accounting for the specifics of the distribution problem at hand, which are subjects' chances and potential outcomes in our investigation.

\subsection{Concluding remarks}

In this paper we have used a controlled laboratory experiment to investigate the allocations of non-involved individuals in distribution problems with and without uncertainty. We observe that allocations in distribution problems characterized by uncertainty are highly dispersed compared to a situation where there is no uncertainty on the final distribution of resources. Four fairness ideals are theoretically endorsed: ex-ante and ex-post equality, utilitarianism and equity. We find that each of the four ideals fits a significant

\footnotetext{
${ }^{21}$ Results are similar, but more significant, when the Pearson correlation coefficient is employed: the mean correlation coefficient is $0.19, p=0.06$, for ex-post fairness, $-0.4, p=0.01$, for ex-ante fairness, $-0.14, p=0.26$, for utilitarianism and $-0.05, p=0.54$, for equity.
} 
share of the observed allocations. More than half of the allocations are motivated by the two fairness ideals inspired by the principle of equality, one third of the allocations are consistent with the equity principle and $15 \%$ with utilitarianism. In accordance with previous findings (see Konow, 2000 and Cappelen, 2011) we observe that Equality is the principle that inspires most allocations. However, we also observe that its interpretation is related to the riskiness of the distribution problem. In particular, we find that ex-post equality concerns are more important than ex-ante equality concerns the higher the riskiness of the distribution problem. Hence, people not only hold different ideas about fairness, but these ideas also depend on the characteristics of the situation. We also find that individuals believe that others have similar risk preferences as their own ones and that such preferences influence allocation decisions. In particular, the higher one's aversion to risk the more money is allocated to the subject exposed to uncertainty.

Risk and ambiguity are often found to elicit quite different behavior in individual choice problems. We do not observe any significant effect of these forms of uncertainty on Benevolent Dictators allocation decisions. We suggest two explanations: first, the possible outcomes of the ambiguous distribution problems in our experiment are the same as the outcomes that have a 50\% chance in some of the risky problems we employ. This feature of our experimental design may have induced subjects to think of the ambiguous event as a 50-50 prospect. Secondly, when people make decision from an external viewpoint they act in a normative way and hence treat ambiguity according to the principle of insufficient reason. This latter interpretation is supported by the fact that altogether allocation behavior is largely consistent with normative ideas of fairness derived from the theory of distributive justice. More research is however needed to confirm this interpretation.

The observed heterogeneity of fairness ideals helps explaining why controversies are common in many allocation problems where uncertainty affects people's final outcomes. Disagreement may stem from a normatively different perspective, that opposes, for instance, utilitarian objectives to a compensation of the weaker principle. More importantly, our results show that even though the majority of people is motivated by the ideal of treating equally deserving people in the same way, the presence of uncertainty leads to different interpretations of the concept that are taken up in a con- 
text dependent way. Some empirical studies show that when people have stakes in a distribution problem they tend to interpret fairness in a way that is most beneficial to their own selfish ends (see Babcock et al. 1996, Babcock and Loewenstein, 1997 and Rodriguez-Lara and Moreno-Garrido, 2011). Further, self-serving biases seem to emerge especially when information on the relation between actions and outcomes can be selectively chosen or interpreted (see, for instance, Dana et al., 2007). Thus, a natural and interesting continuation of our study would be to investigate stakeholders' fairness views and allocation behavior in distribution problems of the type exposed in this paper. Our results provide a necessary preliminary step in this direction.

\subsection{Appendix}

\subsubsection{Fairness ideals: analytical derivation}

\section{Egalitarianism}

$$
\min _{x_{R}^{m}, x_{C}^{m}} p_{e}\left|k_{R} x_{R}^{m}-x_{C}^{m}\right|+\left(1-p_{e}\right)\left|\bar{k}_{R} x_{R}^{m}-x_{C}^{m}\right| \text { s.t. } x_{R}^{m}+x_{C}^{m}=X
$$

In order to simplify notation we omit the superscript $m$ which indicates the decision problem. Substituting the constraint into the equation to minimize we obtain:

$$
\begin{array}{r}
\min _{x_{R}} p_{e}\left|k_{R} x_{R}-\left(X-x_{R}\right)\right|+\left(1-p_{e}\right)\left|\bar{k}_{R} x_{R}-\left(X-x_{R}\right)\right| \\
\left.\min _{x_{R}} p_{e} \mid x_{R}\left(k_{R}+1\right)-X\right)\left|+\left(1-p_{e}\right)\right| x_{R}\left(\bar{k}_{R}+1\right)-X \mid
\end{array}
$$

case $1 \quad x_{R} \geq \frac{X}{\bar{k}_{R}+1}\left(\Rightarrow x_{R} \geq \frac{X}{k_{R}+1}\right)$

$$
\begin{array}{r}
\left.\min _{x_{R}} p_{e}\left[x_{R}\left(k_{R}+1\right)-X\right)\right]+\left(1-p_{e}\right)\left[x_{R}\left(\bar{k}_{R}+1\right)-X\right] \\
\frac{\partial}{\partial x_{R}}=p_{e}\left(k_{R}+1\right)+\left(1-p_{e}\right)\left(\bar{k}_{R}+1\right)>0 \Rightarrow \\
x_{R}^{1}=\frac{X}{\bar{k}_{R}+1}
\end{array}
$$


case $2 \quad x_{R} \leq \frac{X}{k_{R}+1}\left(\Rightarrow x_{R} \leq \frac{X}{\bar{k}_{R}+1}\right)$

$$
\begin{array}{r}
\left.\min _{x_{R}} p_{e}\left[X-x_{R}\left(k_{R}+1\right)\right)\right]+\left(1-p_{e}\right)\left[X-x_{R}\left(\bar{k}_{R}+1\right)\right] \\
\frac{\partial}{\partial x_{R}}=-p_{e}\left(k_{R}+1\right)-\left(1-p_{e}\right)\left(\bar{k}_{R}+1\right)<0 \Rightarrow \\
x_{R}^{2}=\frac{X}{k_{R}+1}
\end{array}
$$

case $3 \quad \frac{X}{k_{R}+1} \leq x_{R} \leq \frac{X}{\bar{k}_{R}+1}$

$$
\begin{array}{r}
\min _{x_{R}} p_{e}\left[x_{R}\left(k_{R}+1\right)-X\right]+\left(1-p_{e}\right)\left[X-x_{R}\left(\bar{k}_{R}+1\right)\right] \\
\frac{\partial}{\partial x_{R}}=p_{e}\left(k_{R}+1\right)-\left(1-p_{e}\right)\left(\bar{k}_{R}+1\right) \\
\text { given that } \frac{p_{e}}{1-p_{e}}>\frac{\bar{k}_{R}+1}{k_{R}+1} \forall p_{e}, k_{R}, \bar{k}_{R} \Rightarrow \frac{\partial}{\partial x_{R}}>0 \\
x_{R}^{3}=\frac{X}{k_{R}+1}
\end{array}
$$

Since the function to minimize is continuous in $x_{R}$, increasing for $x_{R} \geq$ $\frac{X}{k_{R}+1}$ and decreasing for $x_{R} \leq \frac{X}{k_{R}+1}$, it follows that the global minimum is:

$$
x_{R}^{E g l}=\frac{X}{k_{R}+1} \quad x_{C}^{E g l}=\frac{X k_{R}}{k_{R}+1}
$$

\section{Sen's weak equity axiom}

$$
E\left[U\left(x_{R}\right)\right]=E\left[U\left(x_{C}\right)\right] \text { s.t. } x_{R}+x_{C}=X
$$

Assuming that $U(x)=x^{\alpha}$ we can rewrite the previous equations as:

$$
p_{e}\left(x_{R} k_{R}\right)^{\alpha}+\left(1-p_{e}\right)\left(x_{R} \bar{k}_{R}\right)^{\alpha}=\left(x_{C}\right)^{\alpha}
$$




$$
\begin{aligned}
p_{e} x_{R}^{\alpha} k_{R}^{\alpha}+\left(1-p_{e}\right) x_{R}^{\alpha} \bar{k}_{R}^{\alpha} & =\left(X-x_{R}\right)^{\alpha} \\
x_{R}^{\alpha}\left(p_{e} k_{R}^{\alpha}+\left(1-p_{e}\right) \bar{k}_{R}^{\alpha}\right) & =\left(X-x_{R}\right)^{\alpha} \\
\ln x_{R}^{\alpha}+\ln \left(p_{e} k_{R}^{\alpha}+\left(1-p_{e}\right) \bar{k}_{R}^{\alpha}\right) & =\ln \left(X-x_{R}\right)^{\alpha}
\end{aligned}
$$

By setting $\ln \left[p_{e} k_{R}^{\alpha}+\left(1-p_{e}\right) \bar{k}_{R}^{\alpha}\right]=Z$ we can rewrite the previous equation as:

$$
\begin{aligned}
\alpha \ln x_{R}+Z & =\alpha \ln \left(X-x_{R}\right) \\
\alpha\left(\ln x_{R}-\ln \left(X-x_{R}\right)\right) & =-Z \\
\alpha \ln \frac{x_{R}}{X-x_{R}} & =-Z \\
\frac{x_{R}}{X-x_{R}} & =e^{\frac{-Z}{\alpha}} \\
\frac{X}{x_{R}}-1 & =e^{\frac{Z}{\alpha}}
\end{aligned}
$$

Hence, the fair allocations to $R$ and $C$ are:

$$
x_{R}^{S e n}=\frac{X}{e^{\frac{Z}{\alpha}}+1} \quad x_{C}^{S e n}=\frac{X e^{\frac{Z}{\alpha}}}{e^{\frac{Z}{\alpha}}+1}
$$

\section{Utilitarianism}

$$
\max _{x_{R}, x_{C}} E\left[U\left(x_{R}\right)+U\left(x_{C}\right)\right] \text { s.t. } x_{R}+x_{C}=X
$$

Assuming that $U(x)=x^{\alpha}$ we can rewrite the previous equations as:

$$
\max _{x_{R}} p_{e}\left(x_{R} k_{R}\right)^{\alpha}+\left(1-p_{e}\right)\left(x_{R} \bar{k}_{R}\right)^{\alpha}+\left(X-x_{R}\right)^{\alpha}
$$


Consider the case where $0<\alpha<1$. The first order condition is then:

$$
\frac{\partial}{\partial x_{R}}=\alpha p_{e} k_{R}\left(x_{R} k_{R}\right)^{\alpha-1}+\left(1-p_{e}\right) \alpha \bar{k}_{R}\left(x_{R} \bar{k}_{R}\right)^{\alpha-1}-\alpha\left(X-x_{R}\right)^{\alpha-1}=0
$$

$$
\begin{aligned}
x_{R}^{\alpha-1}\left(p_{e} k_{R}^{\alpha}+\left(1-p_{e}\right) \bar{k}_{R}^{\alpha}\right) & =\left(X-x_{R}\right)^{\alpha-1} \\
\ln x_{R}^{\alpha-1}+\ln \left(p_{e} k_{R}^{\alpha}+\left(1-p_{e}\right) \bar{k}_{R}^{\alpha}\right) & =\ln \left(X-x_{R}\right)^{\alpha-1}
\end{aligned}
$$

By setting $\ln \left[p_{e} k_{R}^{\alpha}+\left(1-p_{e}\right) \bar{k}_{R}^{\alpha}\right]=Z$ we can rewrite the previous equation as:

$$
\begin{aligned}
\ln x_{R}^{\alpha-1}+Z & =\ln \left(X-x_{R}\right)^{\alpha-1} \\
(\alpha-1) \ln x_{R}+Z & =(\alpha-1) \ln \left(X-x_{R}\right) \\
\ln \frac{X-x_{R}}{x_{R}} & =\frac{Z}{\alpha-1} \\
\frac{X}{x_{R}}-1 & =e^{\frac{Z}{\alpha-1}}
\end{aligned}
$$

The solution of the maximization problem leads to the following fair allocations:

$$
x_{R}^{U t i l}=\frac{X}{e^{\frac{Z}{\alpha-1}}+1} \quad x_{C}^{U t i l}=\frac{X e^{\frac{Z}{\alpha-1}}}{e^{\frac{Z}{\alpha-1}}+1}
$$

Consider now the case where $\alpha>1$. The function to maximize is convex and thus the solution to the maximization problem is:

$$
x_{R}^{U t i l}=X \quad x_{C}^{U t i l}=0
$$




\subsubsection{Answers in the debriefing questionnaire}

In the following we provide some examples of the answers provided by Benevolent Dictators in the debriefing questionnaire, where they were asked to shortly explain their allocation decisions. Answers are grouped into four categories that correspond to the theoretically identified fairness ideals.

Examples of answers related to ex-ante equality:

"tried to give B a bit more as he has the risk."

"compensate B with a higher amount to compensate the risk of him getting 0 "

"Since B takes a higher risk, he/she deserves a higher payout to remedy the risk he/she takes"

Examples of answers related to ex-post equality:

"Since it would be either 5 times the amount or nothing I wanted to let the amount that B could earn be equal that of C. So I gave 3 points to $\mathrm{B}$ (so that this person could earn 15 Euro) and the remaining 13 to $\mathrm{C}$. This way the least amount of points was 'wasted' and could lead to an equal distribution."

"in case B is rewarded with money, the amount will be multiplied by 5 . I chose this distribution in order for everyone to have almost the same outcome"

"My aim was that if B wins, B wont earn much more than C."

Examples of answers related to utilitarianism:

"Since B has greater possibilities to get 0 . I allocate more to C" "The chance was low for B to win, therefore more money to C."

Examples of answers related to equity:

"I wanted to give $\mathrm{B}$ and $\mathrm{C}$ what they earned in their assignment, so 8 per person. $50 \%$ chance of 16 Euro and $50 \%$ chance of 0 Euro will also lead to a average earning of 8 (when repeating it very often)"

"Both scored the same amount of money. I did not want to punish $\mathrm{C}$ for being selected as $\mathrm{C}$ “ 
"I always allocated 8 because I thought this was most fair for $\mathrm{C}$ who always got the amount allocated. $\mathrm{C}$ earned 8 so I thought it was good to always grant him that amount. B had some random events that also influenced his earnings but since I could not influence those, I didn't take it into account"

\subsubsection{Instructions of the experiment}

\section{Introduction speech}

In this experiment you can earn money with the decisions you make. Your earnings may also depend on chance events and the decisions of other participants. At the end of the experiment you will be paid out in cash individually and confidentially. In order to ensure the highest level of anonymity and confidentiality, the payment will be carried out by a person that is not involved in this research project. The experimenters cannot link your earnings and decisions to your identity in any way. During the experiment you are not allowed to communicate in any other way than described in the instructions. If you have any questions please raise your hand. An experimenter will then come to you and answer your questions in private. The experiment consists of 3 parts. You will receive the instructions of a part only after the previous part has ended.

\section{Part 1}

In the first part of the experiment you will be randomly matched into groups of three participants, which will be labeled with the letters A, B and C. In this part of the experiment the $\mathrm{B}$ and $\mathrm{C}$ members of a group are asked to independently perform a task that involves correctly positioning sliders on a bar. Below you can see the representation of a slider in the initial position a) and in the correct position b), which is always in the middle of the bar. The slider is positioned correctly if the number that shows up to the right of the slider equals 50 .

For each correctly positioned slider 0.25 Euro are credited. There are a total of 32 sliders to be positioned in 6 minutes time, so that $\mathrm{B}$ and $\mathrm{C}$ can be credited up to 8 Euro each. After the 6 minutes are over, the credit accumu- 
Figure 3.4: a) initial position of the slider

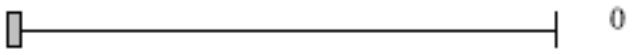

Figure 3.5: b) correct position of the slider

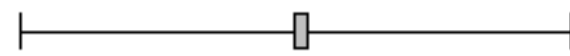

lated by $\mathrm{B}$ and $\mathrm{C}$, who are in the same group, is deposited in a joint group account. Each member of a group (A, B and C) is then informed about the total amount in the joint account of their group. A also receives information about how many sliders were correctly positioned by $\mathrm{B}$ and $\mathrm{C}$ members in her or his group.

The task of $A$ and earnings determination for A, B, and C: Person A earns money for performing a task, which is described below. At the end of the experiment, the earnings of A will be publicly and randomly determined by drawing a card from a stack of numbered cards. The earnings of A can be 4.-, 6.-, 10.- or 12.- Euro and each of these earnings are equally likely. Notice that the earnings of A only depend on chance. In particular, the earnings of A do in no way depend on the decisions taken by A. Also notice that the earnings of A are not taken from the joint account.

The task of $\mathrm{A}$ is to divide the amount of money in the joint account between $\mathrm{B}$ and C. A is asked to make a division in 7 different decision situations. At the end of the experiment one out of the 7 decisions will be randomly selected to determine $\mathrm{B}$ and $\mathrm{C}$ earnings. Each decision situation is independent and equally likely to be the one that determines the earnings of $\mathrm{B}$ and C. Therefore, person A should carefully consider each decision and make each decision in isolation.

The 7 decision situations differ in the way the amount of money assigned to $\mathrm{B}$ and $\mathrm{C}$ translates into earnings for $\mathrm{B}$ and $\mathrm{C}$. The table below summarizes the 7 decision situations and shows how the earnings of $\mathrm{B}$ and $\mathrm{C}$ are determined in each decision situation. Notice that during the experiment the 7 decision situations will appear in random order. Please have a look at it. 
3.6. Appendix

\begin{tabular}{lll}
\hline & Earnings of B & Earnings of C \\
\hline \hline 1 & allocation to B & allocation to C \\
2 & $20 \%$ chance 5 times allocation to B, $80 \%$ times 0 & allocation to C \\
3 & $50 \%$ chance 2 times allocation to B, $50 \%$ times 0 & allocation to C \\
4 & $80 \%$ chance 1.25 times allocation to B, $20 \%$ times 0 & allocation to C \\
5 & $50 \%$ chance 1.5 times allocation to B, $50 \%$ times 0.5 times allocation to B & allocation to C \\
6 & unknown chance 2 times allocation to B, unknown chance 0 & allocation to C \\
7 & unknown chance 1.5 times allocation to B, unknown chance 0.5 times allocation to B & allocation to C \\
\hline
\end{tabular}

We will now explain each decision situation in detail.

If, at the end of the experiment, decision situation 1 is selected to matter for payment then the earnings of $\mathrm{B}$ are equal to the allocation to $\mathrm{B}$ and the earnings of $\mathrm{C}$ are equal to the allocation to $\mathrm{C}$.

If, at the end of the experiment, decision situation 2 is selected to matter for payment the final earnings of B depend on the amount of Euro allocated to $B$ and on a chance event. The chance event will be the public drawing of a card from a stack of 100 cards numbered from 1 to 100 . If a card with a number from 1 to 20 will be drawn then the earnings of $B$ will be 5 times the money allocated to B (i.e., $500 \%$ of the allocation to B). If a number from 21 to 100 will be drawn then the earnings of $B$ will be 0 Euro. In other words, with $20 \%$ chance the earnings of $\mathrm{B}$ will be 5 times the allocation to $\mathrm{B}$ and with $80 \%$ chance the earnings of $\mathrm{B}$ will be 0 Euro. The earnings of $\mathrm{C}$ are equal to the allocation to $\mathrm{C}$.

If, at the end of the experiment, decision situation 3 is selected to matter for payment the final earnings of B depend on the amount of Euro allocated to $\mathrm{B}$ and on a chance event. The chance event will the public drawing of a card from a stack of 100 cards numbered from 1 to 100. If a card with a number from 1 to 50 will be drawn then the earnings of $B$ will be 2 times the allocation to B (i.e. $200 \%$ of the allocation to B). If a number from 51 to 100 will be drawn then the earnings of $B$ will be 0 Euro. In other words, with $50 \%$ chance the earnings of $\mathrm{B}$ will be 2 times the allocation to $\mathrm{B}$ and with $50 \%$ chance the earnings of $B$ will be 0 Euro. The earnings of $C$ are equal to the allocation to $\mathrm{C}$. 
If, at the end of the experiment, decision situation 4 is selected to matter for payment the final earnings of B depend on the amount of Euro allocated to $\mathrm{B}$ and on a chance event. The chance event will the public drawing of a card from a stack of 100 cards numbered from 1 to 100 . If a card with a number from 1 to 80 will be drawn then the earnings of $\mathrm{B}$ will be 1.25 times the allocation to B (i.e. $125 \%$ of the allocation to B). If a number from 81 to 100 will be drawn then the earnings of $B$ will be 0 Euro. In other words, with $80 \%$ chance the earnings of $\mathrm{B}$ will be 1.25 times the allocation to $\mathrm{B}$ and with $20 \%$ chance the earnings of $\mathrm{B}$ will be 0 Euro. The earnings of $\mathrm{C}$ are equal to the allocation to $\mathrm{C}$.

If, at the end of the experiment, decision situation 5 is selected to matter for payment the final earnings of B depend on the amount of Euro allocated to $\mathrm{B}$ and on a chance event. The chance event will the public drawing of a card from a stack of 100 cards numbered from 1 to 100. If a card with a number from 1 to 50 will be drawn then the earnings of $B$ will be 1.5 times the allocation to B (i.e. $150 \%$ of the allocation to B). If a number from 51 to 100 will be drawn then the earnings of $B$ will be 0.5 times the allocation to $\mathrm{B}$ (i.e. $50 \%$ of the allocation to B). In other words, with $50 \%$ chance the earnings of $B$ will be 1.5 times the allocation to $B$ and with $50 \%$ chance the earnings of $\mathrm{B}$ will be 0.5 times the allocation to $\mathrm{B}$. The earnings of $\mathrm{C}$ are equal to the allocation to $\mathrm{C}$.

If, at the end of the experiment, decision situation 6 is selected to matter for payment the final earnings of B depend on the amount of Euro allocated to $\mathrm{B}$ and on a chance event. The experimenters will first randomly select black or red to be the winning color. The chance event will then be the public drawing of a card from a stack of 100 cards which are black or red. The total number of red and black cards sums up to 100, but neither A nor $\mathrm{B}$ nor $\mathrm{C}$ nor the experimenters know how many red cards and how many black cards are in the stack. If a card with the winning color is drawn the earnings of $\mathrm{B}$ will be 2 times the allocation to $\mathrm{B}$ (i.e. $200 \%$ of the allocation to $B$ ). If a card with the losing color is drawn then the earnings of $B$ will be 0 Euro. In other words, with an unknown chance the earnings of $B$ will be 2 times the allocation to $B$ and with an unknown chance the earnings of $B$ will be 0 Euro. The earnings of $\mathrm{C}$ are equal to the allocation to $\mathrm{C}$. 
If, at the end of the experiment, decision situation 7 is selected to matter for payment the final earnings of B depend on the amount of Euro allocated to $B$ and on a chance event. The experimenters will first randomly select black or red to be the winning color. The chance event will then be the public drawing of a card from a stack of 100 cards which are black or red. The total number of red and black cards sums up to 100, but neither A nor B nor C nor the experimenters know how many red cards and how many black cards are in the stack. If a card with the winning color is drawn the earnings of $\mathrm{B}$ will be 1.5 times the allocation to $\mathrm{B}$ (i.e. $150 \%$ of the allocation to $\mathrm{B}$ ). If a card with the losing color is drawn then the earnings of $\mathrm{B}$ will be 0.5 times the allocation to B (i.e. $50 \%$ of the allocation to B). In other words, with an unknown chance the earnings of $B$ will be 1.5 times the allocation to $B$ and with an unknown chance the earnings of $\mathrm{B}$ will be 0.5 times the allocation to $\mathrm{B}$. The earnings of $\mathrm{C}$ are equal to the allocation to $\mathrm{C}$.

If you have any question please raise your hand and an experimenter will come to answer your question in private. In the following you are asked a few questions that will help us assessing your understanding of the decision situations described above. Please fill in the missing figures. Note, that in these questions we are not interested in the actual numbers you fill in but only if you fill them in correctly. During the experiment you will have the possibility to use a calculator by clicking on the icon in the bottom right corner of the screen. When you are ready please raise your hand and an experimenter will come to you to check your answers. Once you are ready please wait quietly.

Consider decision situation 3 and assume that the total in the joint account is 16 Euro. If $\mathrm{A}$ assigns ... Euro to $\mathrm{C}$ and ... Euro to B, then this means that with...\% chance B earns ... Euro and with ...\% chance ...Euro. C earns .... Euro.

Consider decision situation 5 and assume that the total in the joint account is 15 Euro. If A assigns ... Euro to $\mathrm{C}$ and ... Euro to B, then this means that with ...\% chance B earns ... Euro and with ...\% chance ... Euro. C earns .... Euro. 
Consider decision situation 7 and assume that the total in the joint account is 12 Euro. If A assigns ... Euro to $\mathrm{C}$ and ... Euro to B, then this means that with ...\% chance B earns ... Euro and with ...\%chance ...Euro. C earns ... Euro.

\section{Part 2}

You are now going to make a series of decisions. These decisions will not influence your earnings from the first part of the experiment, nor will the decisions you made in the first part of the experiment influence the earnings from this part. Furthermore, the decisions you are going to make will only influence your own earnings.

You will be confronted with 12 decision situations. All these decision situations are completely independent of each other. A choice you made in one decision situation does not affect any of the other following decision situations.

Each decision situation is displayed on a screen. The screen consists of 20 rows. You have to decide for every row whether you prefer option A or option B. Option A is the same for every row in a given decision situation, while option B takes 20 different values, one for each row. Note that within a decision situation you can only switch once from option B to option A: if you switch more than once a warning message will appear on the screen and you will be asked to change your decisions. By clicking on NEXT you will see some examples screens of decision situations.

This is a screen shot of a typical decision situation that you are going to face. You are not asked to make choices now! Please have a careful look. Thereafter click on NEXT to proceed.

\section{[INSERT SCREENSHOT 1 HERE]}

This is another screen shot of a typical decision situation that you are going to face. If you want to review the previous example click on BACK, otherwise click on NEXT to proceed. 
Determination of earnings At the end of the experiment one of the 12 decision situations will be randomly selected with equal probability. Once the decision situation is selected, one of the 20 rows in this decision situation will be randomly selected with equal probability. The choice you have made in this specific row will determine your earnings.

Consider, for instance, the first screen shot that you have seen. Option A gives you a $25 \%$ chance to earn 16 .- Euro and a $75 \%$ chance to earn 4 .- Euro. Option B is always a sure amount that ranges from 16.- Euro in the first row, to 4.60 Euro in the 20 th row. Suppose that the 12 th row is randomly selected. If you would have selected option B, you would receive 9.40 Euro. If, instead, you would have selected option A, the outcome of the lottery determines your earnings. At the end of the experiment the lottery outcome will be publicly determined by randomly drawing a card from a stack of numbered cards. Consider now the second screen shot that you have seen. Option A gives you an unknown chance to earn 12.- Euro and an unknown chance to earn 4.Euro. Option B is always a lottery that gives you different chances to earn 12.- Euro or 4.- Euro. Suppose that the 10th row is randomly selected. If you would have selected option B, you would receive 12.- Euro with $55 \%$ chance and 4.- Euro with $45 \%$ chance. If, instead, you would have selected option A, a stack of red and black cards would be used at the end of the experiment to determine whether you earn 12.- Euro or 4.- Euro. This stack of cards will be the same that has been described in part 1: recall that the exact number of black cards and the exact number of red cards in the stack are unknown to you and to us as well. You would earn 12.- Euro if a card of the winning color is drawn and 4.- Euro otherwise.

Please note that each decision situation has the same likelihood to be the one that is relevant for your earnings. Therefore, you should view each decision independently and consider all your choices carefully. If you like to, you can review the examples screens once more by clicking on BACK. If you have any questions please raise your hand. When you are ready, please press the BEGIN button below. 


\section{Part 3}

In the following you are asked to estimate the choices made by one of your group members in 6 decision situations of the second part of the experiment. After having made these estimates you will answer a questionnaire and then the experiment will be over.

You are going to be randomly matched to one of your group members. For a certain decision situation you are asked to indicate which is the last row where you believe your matched group member chooses option B before switching to option A. You earn 1 Euro if you correctly indicate the switching point of your matched group member in a certain decision situation. Therefore, you can earn up to 6 Euro in total. If the true switching point of your matched group member is different from the point you indicated you earn nothing.

If you do not want to indicate a single switching point you can indicate a range of values where you think the switching point of your matched group member lies. If the true switching point lies in this range of values you will earn a positive amount smaller than 1 Euro. The exact amount you earn is calculated according to a formula. The formula captures the idea that earnings are inversely related to the length of the interval you indicate. This means that the larger the interval you indicate the smaller your potential earnings are. This formula also guarantees that your earnings are maximized if you truthfully indicate your estimate. If the true switching point of your counterpart lies outside the interval you indicate you earn nothing. Please click on NEXT to view an example.

This is a screen shot of a typical screen that you are going to see. Assume, for instance, that you believe that your matched group member chooses option B for the last time when option B is equal to 6.- Euro. In such a case, you would type the number 6 in both boxes at the bottom of the screen.

Assume now that you believe that your matched group member may switch from option B to option A when option B takes any value between 8.- Euro and 4.50 Euro. In such a case, you would type the number 8 in the first box and the number 4.50 in the second box. Notice that you earn nothing if you type in two values that cover all possible switching points, in this case if you type in 10 and 0.50 . 
If you have any question please raise your hand. Otherwise click on NEXT to proceed.

\section{[INSERT SCREENSHOT 3 HERE]}

This is another screen shot of a typical screen that you are going to face. Assume, for instance, that you believe that your matched group member chooses option B for the last time when option B gives a chance of $40 \%$ to win 12.- Euro. In such a case, you would type the number 40 in both boxes at the bottom of the screen.

Assume now that you believe that your matched group member switches from option B to option A when the winning chance of option B is between $70 \%$ and $25 \%$. In such a case, you would type the number 70 in the first box and the number 25 in the second box. Notice that you earn nothing if you type in two values that cover all possible switching points, that is if you type in 100 and 5 .

\section{[INSERT SCREENSHOT 4 HERE]}

If you have any question please raise your hand. If you want to review the previous examples once more click on BACK. Otherwise, click on BEGIN to start the third part of the experiment. 
Figure 3.6: Screenshot 1

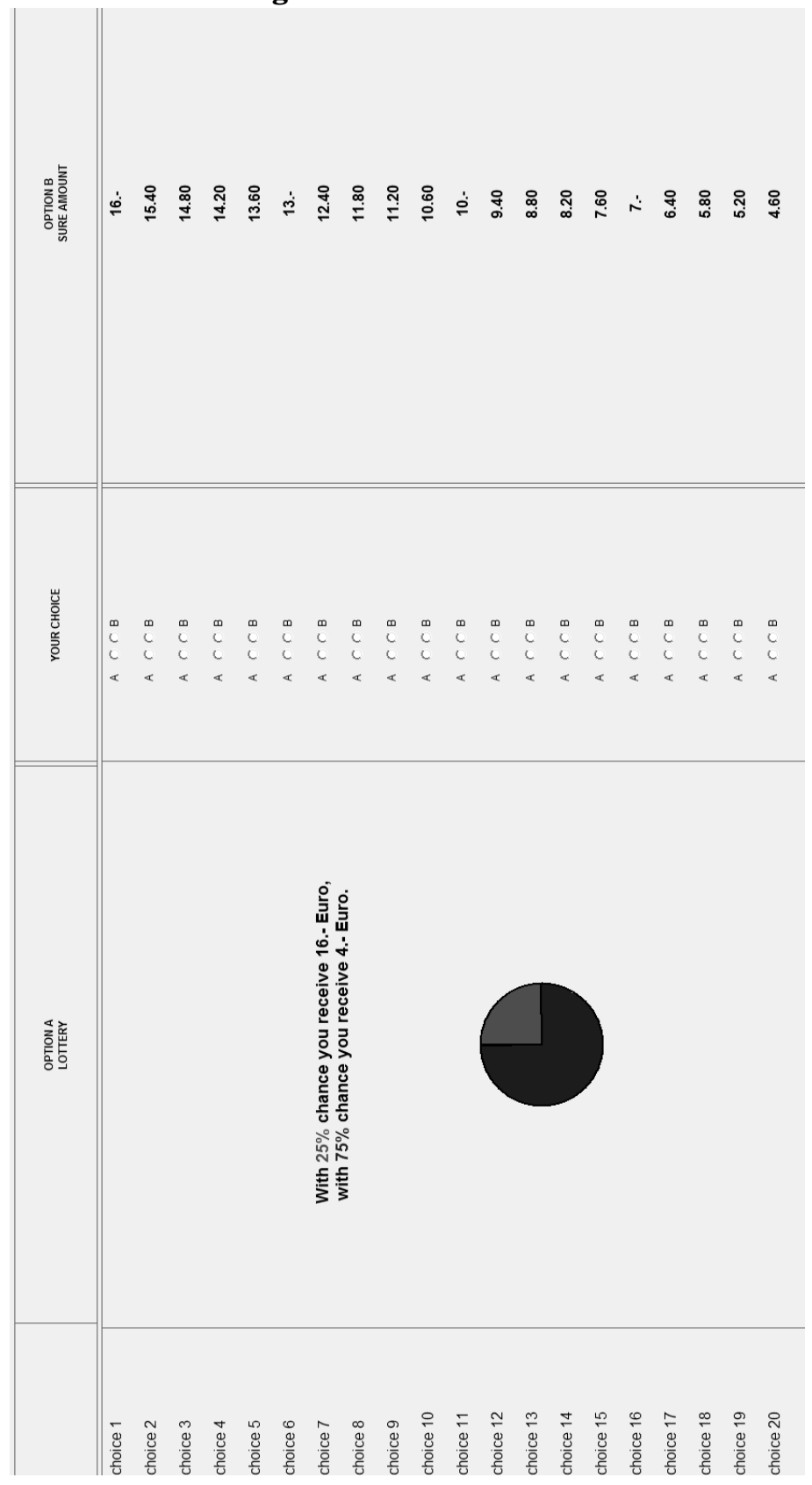


Figure 3.7: Screenshot 2

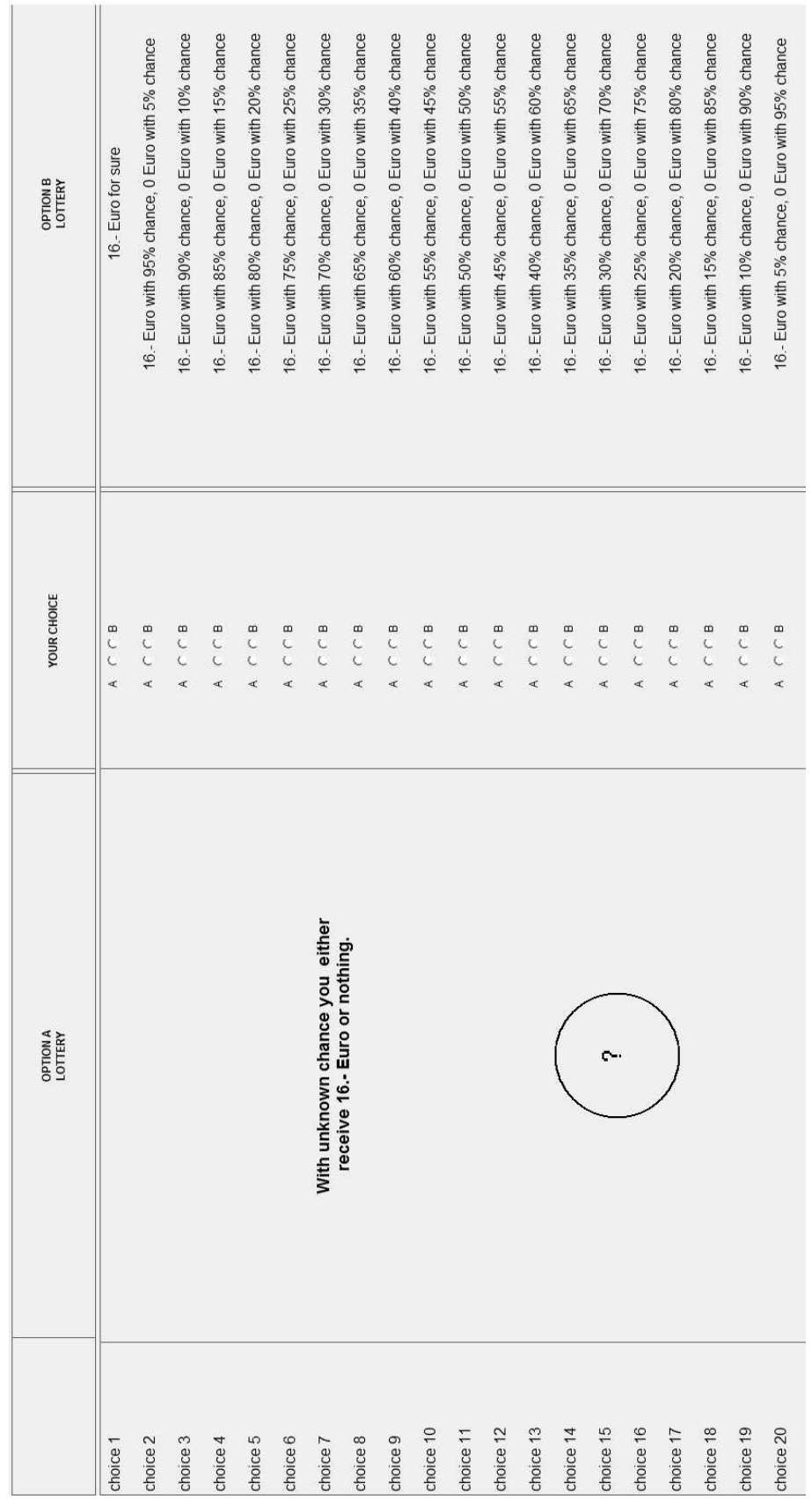


Figure 3.8: Screenshot 3

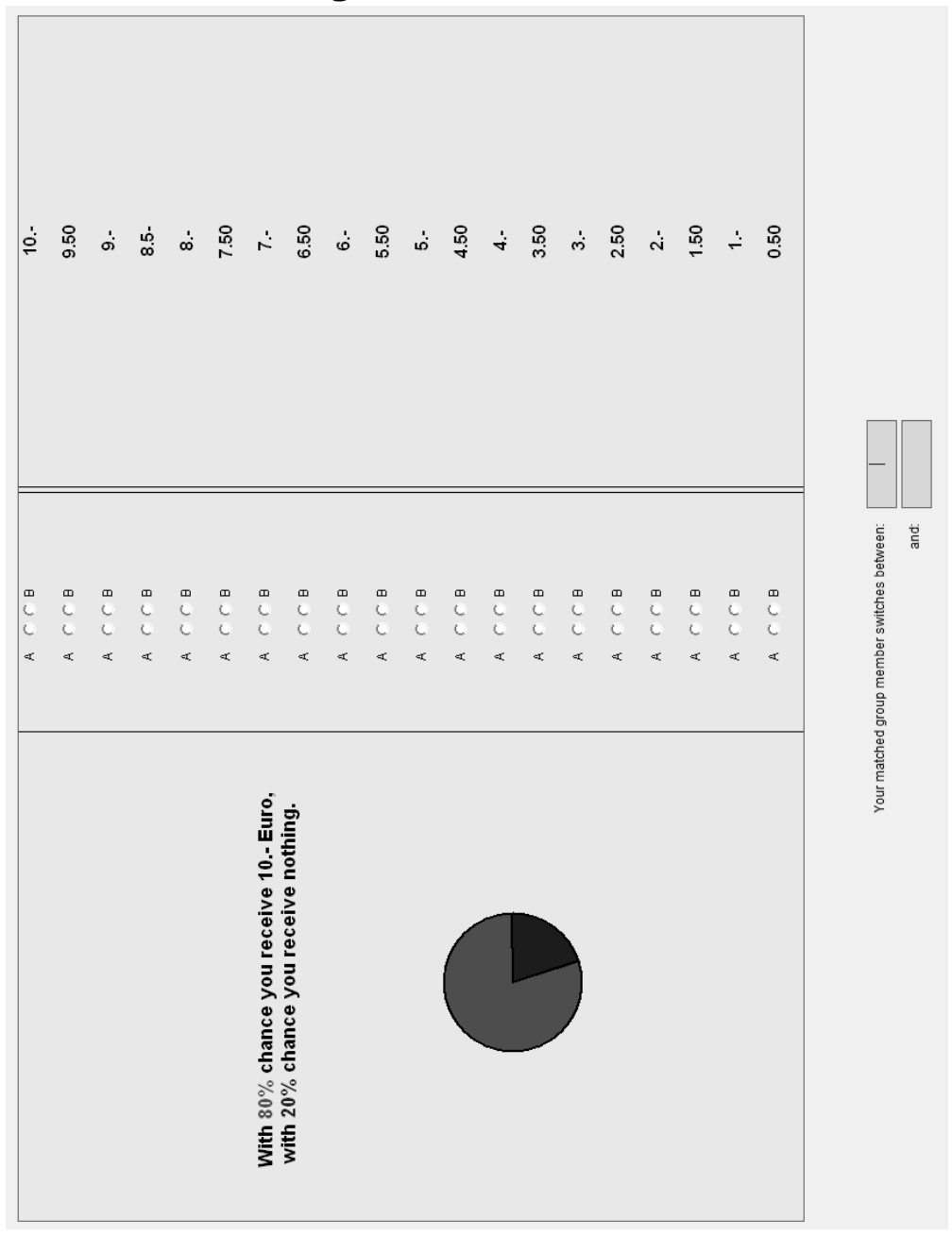


Figure 3.9: Screenshot 4

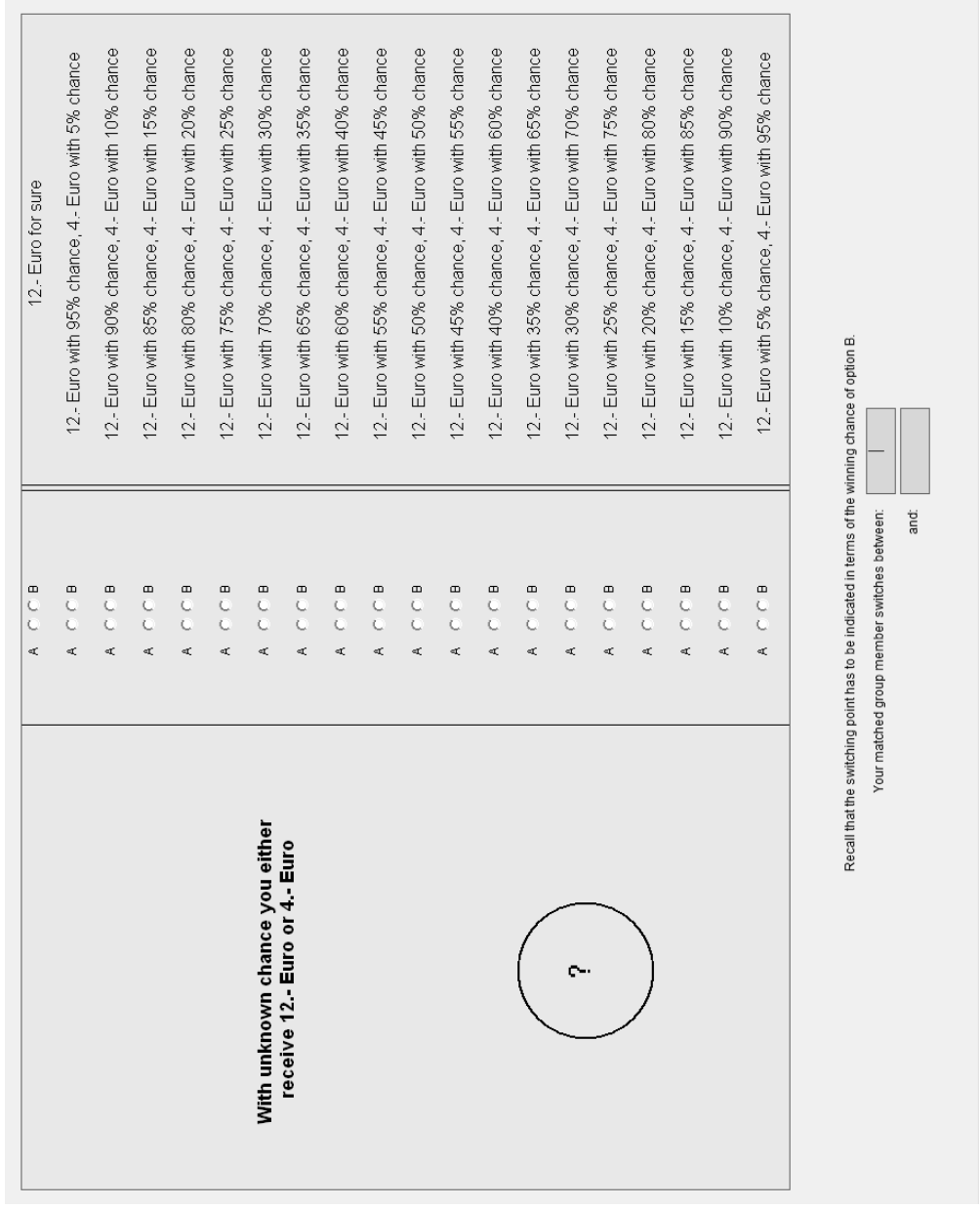





\section{Chapter 4}

\section{Generosity in the Face of Uncertainty}

\subsection{Introduction}

Experimental research has demonstrated that the actions of a large part of the population are inconsistent with the classical assumption of materialistic, self-interested agents (Camerer, 2003). This evidence has been often interpreted as a concern for the well being of others and consequently economists started including sentiments like reciprocity, altruism and inequality aversion into utility analysis. Notwithstanding the fact that expressions like 'social preferences' are by now part of the economic jargon, the robustness of such type of preferences has been recently put into question. Indeed, a few experimental studies have shown that subjects' behavior is sensitive to variations in the environment in a way that is not predicted by interdependent utility models.

For instance, Dana, Cain, and Dawes (2006) demonstrate that roughly one third of individuals prefer to receive $\$ 9$ instead of playing a dictator game over $\$ 10$ with an anonymous recipient. Similarly, Broberg, Ellingsen and Johannesson (2007) elicit reservation prices for exiting the dictator game and find that roughly two-thirds of the subjects are willing to accept less than 100 percent of the dictator endowment in order to opt out. In a variant of the dictator game Dana, Weber and Kuang (2007) show that many dictators 
avoid costless information about the consequences that their decision has on the recipient's payoff and as a result, act more selfishly when compared to the baseline game. Lastly, Haisley and Weber (2010) implement two treatment variations of the dictator game where the recipients' final outcomes could be either subject to risk or ambiguity. They find that otherwise ambiguity averse dictators adopt favorable views of ambiguity and retain on average larger amounts of money than in the risky treatment, even though the objective distribution of outcomes is the same in the risky and in the ambiguous treatment. Altogether these studies suggest that generosity decreases in environments where relevant information can be avoided or interpreted in a self-serving manner. As a consequence, giving behavior in standard dictatorgame experiments may not be the expression of, for instance, a preference for equality. Rather, giving may be the product of internalized sharing norms that constrain people's real goal of pursuing their own material interest (Rabin, 1995). A couple of theoretical papers formally introduce this intuition in decision making models. In Andreoni and Bernheim (2009) giving depends on observability: assuming that in the dictator game the generosity norm is the equal split, the model predicts that the greater the dictator's anonymity the more she can behave selfishly. Konow (2000) develops a model where deviations from a fairness norm are possible through costly self deception. As a consequence, it is predicted that in dictator games individuals act more selfishly the more the experimental set-up allows searching for arguments to support unfair allocations.

In this paper we report the results of an experiment designed to investigate people's generous behavior in situations where the final distribution of outcomes largely depends on events that are uncertain at the time when resources are allocated. In particular, we focus on how people's willingness to sacrifice their own material payoffs to achieve more equitable outcomes depends on their and others' different exposure to uncertainty. The experimental design we employ reproduces the characteristics of many decision situations in the real world. For instance, most of our choices have consequences that span into the future, which is inherently uncertain. Further, although we may know our and others' risk exposure, we cannot know which state out of many risky ones will materialize. Some of the reviewed literature suggests that uncertainty may encourage self-serving interpretations 
of fairness and thereby weaken individuals' willingness to share resources. However, in order to test the self-serving bias hypothesis, we need to account for the fact that in non-standard situations, like the ones we study, norms different than the equal split may apply. If people hold different fairness norms, interpreting giving behavior without taking this heterogeneity into account may not be informative regarding individuals' self-serving tendencies. To address this issue, we exploit the results of the experiment described in Chapter 3 where non-involved subjects had to make allocations in distribution problems with the same characteristics as those employed in this study. The experiment allowed us to identify a set of fairness norms and to estimate their distribution in the population. The existence of self-serving biases is then tested by comparing the distribution of fairness norms among non-involved individuals to the one estimated among involved dictators. ${ }^{1}$

At the beginning of our experiment participants are matched in pairs; one participant is assigned the role of dictator (D), the other the role of recipient (R). Thereafter, both $\mathrm{D}$ and $\mathrm{R}$ individually work on a real effort task by which they earn money that is deposited in a pair-joint account. $\mathrm{D}$ is then asked to allocate the money in the joint account between himself and $\mathrm{R}$ in several distribution problems characterized by different forms of uncertainty, knowing that one will be selected for payment at the end of the experiment. Two treatments are implemented: in the first treatment D always earns exactly what he allocates to himself, while the final earnings of $\mathrm{R}$ are uncertain and can be larger or smaller than his allocation, depending on which state is realized after the division. From now onwards we refer to this treatment as to TUR, that stands for Treatment-Uncertain-Recipient. The converse happens in the second treatment. There, D's final earnings are uncertain while $R$ earns exactly what is allocated to him by $D$. Hence, we refer to this treatment as TUD, Treatment-Uncertain-Dictator. The degree of uncertainty affecting D's or R's final earnings is varied across distribution problems but the expected value of a given allocation stays constant. In the last part of the experiment data on participants' attitude to uncertainty and

\footnotetext{
${ }^{1}$ The importance of accounting for heterogeneous distribution norms to understand giving behavior has been highlighted by some recent papers. See, for example, Cappelen, Sorensen and Tungodden (2007), Cappelen, Konow, Sorensen and Tungodden (2011) and Krupka and Weber (2008).
} 
beliefs about others' attitudes are collected.

We observe that dictators' generosity is independent of whether the dictator himself or the recipient faces earnings' uncertainty. Further, in both treatments dictators are least generous when uncertainty is highest. We find evidence of self-serving biased giving behavior when uncertainty affects dictators' earnings but not when the recipient's earnings are uncertain. Moreover, in the latter case we observe that dictators' generosity increases with their own aversion to risk.

The reminder of the paper is organized as follows. In the following section 4.2 the experimental design is described in detail. In section 4.3 results on dictators' allocation behavior are presented. In section 4.4 fairness norms are discussed and their distribution is estimated. In section 4.5 concluding remarks are drawn. The experiment's instructions are provided in the Appendix.

\subsection{The experiment}

The experiment consists of three parts. In the first part of the experiment subjects are divided in pairs and are randomly assigned the role of Dictator (D) or Recipient (R). Thereafter all subjects work on a real effort task and the money earned by each pair of subjects is deposited in a joint account. In the second part of the experiment every Dictator is asked to split the money in the joint account between himself and the Recipient. In the third and last part, all subjects face a series of incentivized choices that allow us to estimate their preferences for uncertainty and beliefs about others' preferences. In what follows the experiment is described in detail.

Part 1: the real effort task After everyone takes a seat at the designated computer station, participants are informed that the experiment consists of three parts. Thereafter, the instructions for the first and second part are distributed and read aloud by the experimenter. Participants are then randomly matched in groups of two: one group member is assigned the role of Dictator, the other the role of Recipient ${ }^{2}$. In the first part of the experi-

\footnotetext{
${ }^{2}$ In the experiment subjects are assigned the more neutral labels A and B.
} 
ment all subjects work individually on a real effort task. The real effort task consists of a single screen displaying 32 sliders on horizontal bars (Gill and Prowse, 2011). Each slider can be moved at any point of the bar for an unlimited number of times and the actual position of a slider is displayed to the right of the bar. A slider is correctly positioned when the number 50 pops up at the right of the bar, that is when the slider is exactly at the middle of it. Each subject's "score" in the task is the number of sliders positioned at 50 in 6 minutes time. During the task subjects view their current score and the amount of time remaining on the top of the screen. We chose the slider task because it is easy to explain and to understand, identical across repetitions and has no scope for guessing. Performance in the slider task is incentivized. For each correctly positioned slider $€ 0.25$ are credited, so that subjects can credit up to 68 each. After the time for the task has expired, $D$ and $R$ view the performance of the other subject in the pair and the total amount of money generated by such performance. The total amount of money, $X$, is deposited in a pair-joint account. We chose the number of sliders on the screen and the available time to complete the task such that maximum performance is easily achievable. In this way, dictators and recipients in the same group would only differ in their exposure to uncertainty, thereby excluding the possibility that productivity differences affect D's generosity.

Part 2: allocation decisions In the second part of the experiment $D$ has to divide the amount in the group account between himself and $\mathrm{R}$, who is not active in this phase. $\mathrm{D}$ has to make a division in $n=7$ distribution problems. Distribution problem 1-Certainty is the same in both treatments: D's and R's final earnings correspond to the distribution of the money in the joint account made by D. Hence, this distribution problem differs from a standard dictator game only by the fact that the amount to divide is the product of D's and R's efforts in the first part of the experiment. In the following we refer to 1-Certainty also as the baseline distribution problem.

Treatments are different with respect to how uncertainty affects D's and R's earnings. In TUR D's final earnings are equal to the share of $X$ that $D$ allocates to himself, while the final earnings of $R$ depend both on the share allocated to him as well as on the realization of one of two possible 
uncertain events. Conversely, in TUD D's final earnings are subject to uncertainty, while $\mathrm{R}$ earns exactly what has been allocated to him by $\mathrm{D}$. In distribution problems characterized by uncertainty we distinguish between uncertain events whose probability is known, that is risky events, and uncertain events whose likelihood is unknown, that is ambiguous events. We set the parameters such that in all distribution problems the expected value of a given allocation is constant. However, in TUR (TUD) in all distribution problems $n<6$ the earnings of $\mathrm{R}(\mathrm{D})$ are riskier in distribution problem $n+1$ than in distribution problem $n$. Hence, this set up allows studying the effect of different degrees of uncertainty on dictators' generosity without the confounding effects that differences in the expected value of an allocation would introduce. Table 4.1 shows the characteristics of each distribution problem. Consider, for instance, distribution problem 4-Risk. In TUR (TUD) the final earnings of $\mathrm{R}(\mathrm{D})$ are twice his share of $X$ with $50 \%$ chance and are zero with $50 \%$ chance. Distribution problem 7-Ambiguity offers the same possible outcomes: in TUR (TUD) the final earnings of $\mathrm{D}(\mathrm{R})$ may be doubled or may be zero, but this time likelihoods are unknown.

Table 4.1: Distribution problems.

\begin{tabular}{lllll}
\hline & & \multicolumn{2}{c}{ TUR } & \multicolumn{2}{c}{ TUD } \\
Problem & D's earnings & R's earnings & D's earnings & R's earnings \\
\hline 1-Certainty & $y_{1}$ & $X-y_{1}$ & $y_{1}$ & $X-y_{1}$ \\
2-Risk & $y_{2}$ & $0.5:\left(X-y_{2}\right) 1.5,\left(X-y_{2}\right) 0.5$ & $0.5: y_{2} 1.5, y_{2} 0.5$ & $X-y_{2}$ \\
3-Risk & $y_{3}$ & $0.8:\left(X-y_{3}\right) 1.25,\left(X-y_{3}\right) 0$ & $0.8: y_{3} 1.25, y_{3} 0$ & $X-y_{3}$ \\
4-Risk & $y_{4}$ & $0.5:\left(X-y_{4}\right) 2,\left(X-y_{4}\right) 0$ & $0.5: y_{4} 2, y_{4} 0$ & $X-y_{4}$ \\
5-Risk & $y_{5}$ & $0.2:\left(X-y_{5}\right) 5,\left(X-y_{5}\right) 0$ & $0.2: y_{5} 5, y_{5} 0$ & $X-y_{5}$ \\
6-Ambiguity & $y_{6}$ & $p:\left(X-y_{6}\right) 1.5,\left(X-y_{6}\right) 0.5$ & $p: y_{6} 1.5, y_{6} 0.5$ & $X-y_{6}$ \\
7-Ambiguity & $y_{7}$ & $p:\left(X-y_{7}\right) 2,\left(X-y_{7}\right) 0$ & $p: y_{7} 2, y_{7} 0$ & $X-y_{7}$ \\
\hline
\end{tabular}

Note: $y_{n}$ indicates the amount $\mathrm{D}$ keeps for himself, $X-y_{n}$ the amount allocated to $\mathrm{R}$

In the experiment distribution problems appear on the computer screen one at a time. The order of appearance is randomized in each pair level. 
4.2. The experiment

At the end of the experiment one problem is randomly selected to be relevant for payment. For that a stack of cards numbered from 1 to 100 is used to determine earnings in risky distribution problems. For instance, if $\mathrm{R}$ or $\mathrm{D}$ faces a $50 \%$ chance of doubling his allocation, his allocation is eventually doubled only if a card with a number smaller than 51 is drawn. In order to operationalize ambiguity, a stack of 100 cards colored black and red is used. Neither the participants nor the experimenter knows the exact color composition of the stack, and each participant is free to choose his/her winning color at the beginning of the experiment. This procedure is described in details in the instructions that are distributed before the start of the experiment. The determination of earnings took place publicly at the end of the experiment so that subjects could witness how the chance devices were operated.

Part 3: Elicitation of individual characteristics In the last part of the experiment we gather data on individual characteristics that may be related to dictators' allocation decisions.

First of all, we measure risk preferences. Participants' certainty equivalents are elicited for 6 two outcomes lotteries. Table 4.2 shows the outcomes and the probabilities employed. For each lottery subjects see a screen on the computer that contains a description of the lottery and a list of 20 equally spaced sure amounts, ranging from the lottery's highest to lowest outcome. In order to facilitate comprehension, likelihoods are expressed both in percentage points and with a pie chart. In each row of the decision screen subjects have to make a choice between the lottery and the sure amount. Subjects are not allowed to switch back and forth between the sure amount and the lottery, which guarantees a unique switching point. Certainty equivalents are then calculated as the arithmetic mean of the smallest sure amount preferred to the lottery and the next higher sure amount on the list.

We chose the above lotteries in order to measure risk preferences for the range of outcomes that are relevant in the distribution problems of the first part. In particular, note that all the first 4 lotteries in the table have an expected value of $€ 8$ and are characterized by the same probability values that appear in the risky distribution problems. Lottery 5 and 6 provide additional information on risk preferences in the relevant range of outcomes. 
Table 4.2: Lotteries for risk preferences elicitation.

\begin{tabular}{cccr}
\hline \hline Lottery & $p_{1}$ & $x_{1}$ & $x_{2}$ \\
\hline 1 & 0.20 & 40 & 0 \\
2 & 0.50 & 16 & 0 \\
3 & 0.80 & 10 & 0 \\
4 & 0.50 & 12 & 4 \\
5 & 0.25 & 16 & 4 \\
6 & 0.33 & 12 & 0 \\
\hline
\end{tabular}

Note: $p_{1}$ is the probability of winning $€ x_{1}$.

As two distribution problems are characterized by ambiguity, we also elicit subjects' attitude to it. To this end, subjects face 6 decision screens where they make choices between an ambiguous and a series of 20 risky lotteries (see Figure 4.1). All risky lotteries on a decision screen are characterized by the same outcomes pair $x_{1}$ and $x_{2}$, which are those in Table 4.2. The first risky lottery on the list is degenerate and guarantees the high outcome: moving down the list the likelihood of the lottery's high outcome decreases by $5 \%$, while the likelihood of the low outcome increases by $5 \%$. In each decision screen, subjects can switch only once from the risky to the ambiguous lottery. Thus, the switching point provides an interval for a subject's belief about the likelihood of the ambiguous event. As in the first part of the experiment, ambiguity is generated with a deck of red and black cards in unknown color composition.

At the end of the experiment one of the 12 decision screens ( 6 for the elicitation of risk preferences, 6 for the elicitation of ambiguity attitude) and one row within the selected decision screen are randomly selected for payment. The relevant lottery is then publicly played out and earnings are added to those of the first part.

Lastly, we investigate subjects' beliefs about others' risk and ambiguity preferences. In order to do so, we ask subjects to estimate the choices made by the other person in the pair in 4 risky and 2 ambiguous decision screens. Rather than eliciting a point belief, for each of the 6 decision screens a participant is asked to estimate the interval that contains the switching point of 
Figure 4.1: Screen shot of the ambiguity attitude elicitation task.

\begin{tabular}{|c|c|c|c|}
\hline & $\begin{array}{l}\text { OpDIONA } \\
\text { LOIIERY }\end{array}$ & Yоuв сноте & $\begin{array}{l}\text { OPPIONB } \\
\text { LOIIERY }\end{array}$ \\
\hline choice 1 & & A $r C B$ & 16. Euro for sure \\
\hline choice 2 & & $A \subset \subset B$ & 16. Euro vith $95 \%$ chance, 0 Euro with $5 \%$ chance \\
\hline choice 3 & & $A C C B$ & 16. Euro with $90 \%$ chance, 0 Euro with $10 \%$ chance \\
\hline choice 4 & & $A C C B$ & 16. Euro with $85 \%$ chance, 0 Euro with $15 \%$ chance \\
\hline choice 5 & & $A C C B$ & 16. Euro with $80 \%$ chance, 0 Euro with $20 \%$ chance \\
\hline choice 6 & & $A C C B$ & 16.- Euro with $75 \%$ chance, 0 Euro with $25 \%$ chance \\
\hline choice 7 & With unknown chance you either & $A \subset C B$ & 16. Euro with $70 \%$ chance, 0 Euro with $30 \%$ chance \\
\hline choice 8 & & $A C C B$ & 16- Euro with $65 \%$ chance, 0 Euro with $35 \%$ chance \\
\hline choice 9 & & $A C C B$ & 16- Euro with $60 \%$ chance, 0 Euro with $40 \%$ chance \\
\hline choice 10 & & $A C C B$ & 16- Euro with $55 \%$ chance, 0 Euro with $45 \%$ chance \\
\hline choice 11 & & $A C C B$ & 16- Euro with 50\% chance, 0 Euro with $50 \%$ chance \\
\hline choice 12 & & $A \subset C B$ & 16- Euro with $45 \%$ chance, 0 Euro with $55 \%$ chance \\
\hline choice 13 & & $A \subset C B$ & 16. Euro with $40 \%$ chance, 0 Euro with $60 \%$ chance \\
\hline choice 14 & & $A C C B$ & 16- Euro with $35 \%$ chance, 0 Euro with $65 \%$ chance \\
\hline choice 15 & ? & $A C C B$ & 16- Euro vith $30 \%$ chance, 0 Euro with $70 \%$ chance \\
\hline choice 16 & & $A C C B$ & 16. Euro with 25\% chance, 0 Euro with $75 \%$ chance \\
\hline choice 17 & & A CrB & 16- Euro with 20\% chance, 0 Euro with 80\% chance \\
\hline choice 18 & & $A \subset C B$ & 16.- Euro with $15 \%$ chance, 0 Euro with $85 \%$ chance \\
\hline choice 19 & & A $C F B$ & 16.- Euro vith $10 \%$ chance, 0 Euro with $90 \%$ chance \\
\hline choice 20 & & $A \cos \theta$ & 16. Euro with $5 \%$ chance, 0 Euro with $95 \%$ chance \\
\hline
\end{tabular}

the other person. A unique switching point can be indicated as well. The belief elicitation is incentivized using the interval scoring rule (Schlag and Van der Weele, 2009). The rule works as follows: if the true switching point of the other person in the pair lies within the indicated interval, the subject earns an amount that is inversely related to the length of the indicated interval. If the true switching point lies outside the indicated interval, the subject earns nothing. The interval scoring rule has two advantages compared to other scoring rules that either elicit point beliefs or the whole distribution. First, it is less time consuming and cognitively demanding for subjects than, for example, the quadratic scoring rule. Second, it allows inferences that are valid under any degree of subjects' risk aversion and not only when subjects are risk neutral (Schlag and Van der Weele, 2009). After the incentivized tasks subjects are asked a few basic background questions. Thereafter, subjects are paid out confidentially in cash and dismissed.

In total 158 students from Maastricht University participated in the computerized experiment which was conducted in February 2010 and in June 2011 in the Behavioral and Experimental Lab (BEElab) at the Maastricht University School of Business and Economics, using the Z-Tree software (Fischbacher, 2007). 80\% of the subjects were enrolled in the School of Busi- 
ness and Economics and the remaining $20 \%$ were enrolled in other faculties, such as law, medicine and arts. $48 \%$ of the subjects were male. The average age was 24 years. The experiment lasted approximately 75 minutes and the average earnings per subject were $€ 22$.

\subsection{Results}

The real effort task and D's allocation behavior We exclude 6 subjects who revealed in the debriefing questionnaire that they made mistakes in the allocation decisions. ${ }^{3}$ In the production phase, the performance of both $\mathrm{D}$ and $\mathrm{R}$ was maximally in 64 of 71 pairs in TUR and in 67 of 72 pairs in TUD. In the following analysis we focus on those pairs with maximum performance because we want to rule out the effects that differences in production may have on D's allocation decisions. In consequence, in all the analyzed pairs the amount in the joint account is equal to $€ 16$.

The histograms in Figure 4.2 show the distribution of the Euro amount D keeps for him/herself in problem 1-Certainty. The vertical thin line indicates the average amount D keeps. As suggested by the histograms, the amount kept by $\mathrm{D}$ is not significantly different between TUR and TUD (Mann-Whitney test $\mathrm{p}=0.81$ ). This is an expected result given that this distribution problem has the same characteristics in both treatments. The histograms also show that our results are in line with what is usually observed in standard dictator games: dictators never keep less than the equal split, a substantial share of dictators choose the equal split and more than $30 \%$ of the dictators keep all the amount in the joint account.

The histograms in Figure 4.3 show the distribution of the amount D keeps for himself in the 4 distribution problems characterized by risk. In both treatments the mean allocation does not change much with the distribution problems. Indeed, we find no significant differences when pair-wise comparing all distribution problems within a treatment using the Wilcoxon test and the Kolmogorov-Smirnov test. ${ }^{4}$ However in TUR the median amount

\footnotetext{
${ }^{3}$ These subjects allocated more money to the recipient than to themselves in all, or almost all, distribution problems.

${ }^{4}$ The results of all statistical test can be found in the Appendix. We correct for multiple comparisons with the "false discovery rate" method (Benjamini and Hochberg, 1995).
} 
Figure 4.2: Distribution problem 1-Certainty.

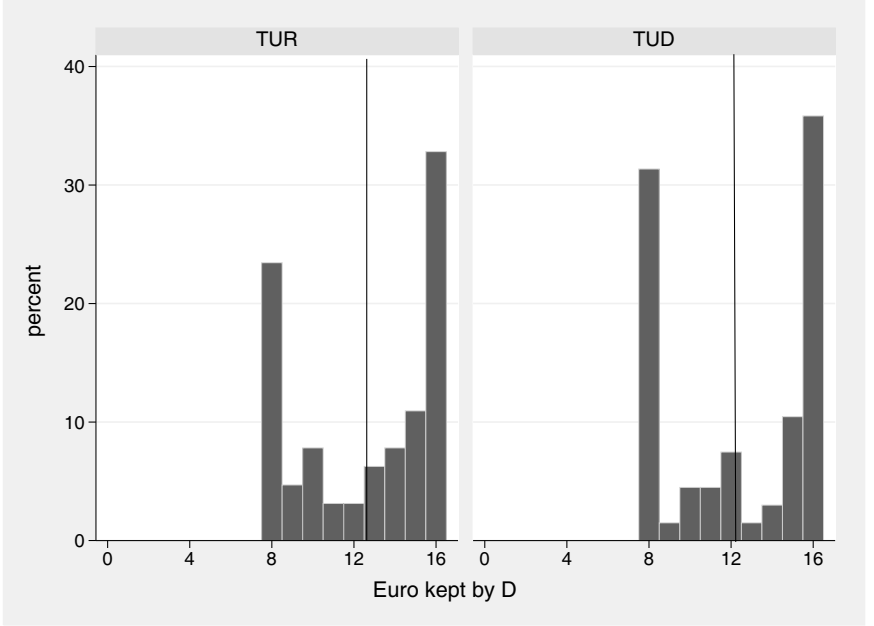

D keeps for himself increases with the riskiness of R's final earnings. The higher the risk the less frequent equal splits are and the more frequently dictators take all the money in the joint account. In TUD D's allocation behavior is not clearly related to the riskiness of the distribution problem. However notice that selfish behavior increases in the distribution problem where D's earnings are subject to the highest risk, that is in 5-Risk. Also notice that differently than in 1-Certainty, in distribution problems characterized by risk some dictators keep less than $€ 8$, the equal split amount.

To further investigate individuals' allocation behavior, we calculate for each $\mathrm{D}$ the Spearman-correlation coefficient between the amount he/she keeps and the rank of the risky problems according to second order stochastic dominance. Figure 4.4 shows how the Spearman-correlation coefficients are distributed in the two treatments. ${ }^{5}$ In TUR the average correlation coefficient is positive (0.24) and significantly different from zero (Wilcoxon test $\mathrm{p}=0.001$ ). Differently stated, in TUR the dictator is less generous the higher the uncertainty of the recipient's final earnings. In TUD the average correlation coefficient is -0.02 , which is not significantly different from zero

\footnotetext{
${ }^{5}$ The high percentage of zeros in both treatments is mainly due to the fact that some dictators keep the whole amount in the joint account in all distribution problems.
} 
Figure 4.3: Distribution problems characterized by uncertainty.

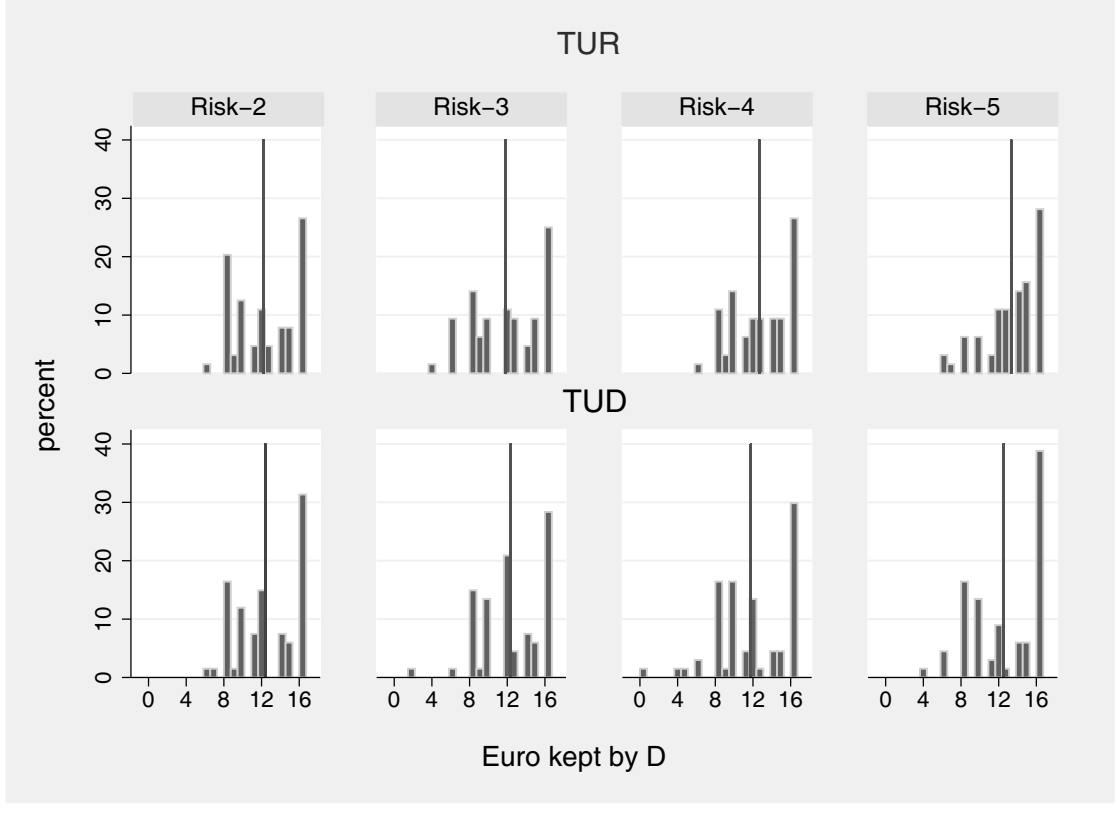

according to the Wilcoxon test.

Result 4.3.1. When recipients' earnings are risky, individuals' generosity is on average inversely related to the earnings' risk. No clear trend is observed when the earnings of the giver are risky. In both type of situations, selfish behavior is most frequent when earnings' risk is highest.

A last observation concerns distribution problems characterized by ambiguity. For a given allocation, 6-Ambiguity and 7-Ambiguity potentially yield the same outcomes as 2-Risk and 4-Risk, respectively. However, the outcomes' likelihoods are known only for the risky problems. Hence, in light of the abundant literature on ambiguity aversion (see Camerer and Weber, 1994) and on the results in Haisley and Weber (2004) reviewed in the Introduction, we hypothesized that dictators would act more selfishly in the ambiguous distribution problems as compared to their risky counterparts. However, in both treatments we observe no statistically significant differences between allocations in ambiguous and risky distribution problems. 
Figure 4.4: Spearman-correlation of D's allocations and distribution problems.

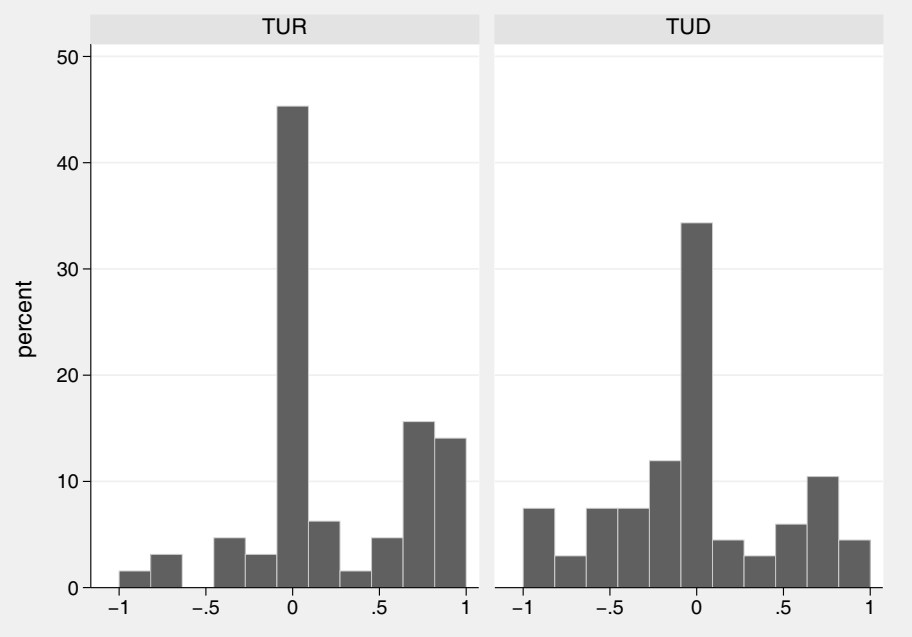

The p-value of the Wilcoxon signed-rank test is at least 0.12.

Individual characteristics In the third part of the experiment participants make several decisions that allow estimating their attitude to risk and ambiguity. As we are interested in investigating possible relations between dictators' preferences for uncertainty and their generous behavior, in the following we exclusively focus on dictators.

First, recall the six decision screens where subjects choose between a risky lottery and a list of varying sure amounts. Using subjects'switching points we calculate dictators' certainty equivalent for each lottery and assume a CRRA utility function for money $U(x)=x^{\alpha}$, where $0<\alpha<1$ indicates risk averse, $\alpha=1$ indicates risk neutral and $\alpha>1$ risk seeking preferences. We then estimate the parameter value of $\alpha$ for each $\mathrm{D}$ by minimizing the sum of squared distances (Wakker, 2008 and Wakker, 2010). That is;

$$
\min _{\alpha} \sum_{i=1}^{6}\left(l_{i}-c e_{i}\right)^{2}
$$

where $l_{i}$ is the expected utility value of lottery $i$ and $c e_{i}$ is the expected utility value of the certainty equivalent of lottery $i$. To correct for heteroscedas- 
ticity prospects are normalized to uniform length. We find that the average $\mathrm{D}$ is characterized by $\alpha=0.79$, ( $\mathrm{d} . \mathrm{d}(\alpha)=0.30$, median $\alpha=0.82$ ), which indicates a moderate aversion to risk.

Second, recall the 6 decision screens where subjects have to make choices between an ambiguous lottery and several risky ones. Assuming Subjective Expected Utility theory (Savage, 1954) choices in these decision screens allow eliciting an interval containing D's prior belief on the ambiguous event. We take the midpoint of this interval to be D's prior belief. As the ambiguous event is always the same in all the decision screens, subjects should consistently reveal the same prior belief in all screens. Pair-wise comparisons of elicited prior beliefs show that subjects indeed hold quite consistent beliefs. ${ }^{6}$ For each D we construct a variable called 'prior-belief' that is the average of the prior beliefs elicited in the six decision screens. Dictators' mean prior-belief is equal to 0.44 (s.d. $=0.1$, median prior-belief $=0.45$ ). That is, on average $\mathrm{D}$ believes that the probability of the ambiguous event is very close to $45 \%$. Since ambiguity aversion can be described as a pessimistically biased belief about the ambiguous event, we conclude that on average $\mathrm{D}$ is only slightly averse to ambiguity. In the last part of the experiment subjects are asked to provide their best estimate of the choices made by the other person in the pair in 4 risky and 2 ambiguous decision screens. Taking the midpoint of the interval indicated by a subject we calculate the believed certainty equivalents for risky lotteries and the believed prior beliefs on the ambiguous event. We find that these beliefs measures are highly and significantly correlated with individuals' own preferences (Spearman's rho $\geq 0.45$, p-value $<0.001$ for all beliefs measures). In order to investigate whether dictators' allocation decisions are related to individuals' observable characteristics we run a regression analysis. As explanatory variables we include distribution problem dummies, a gender dummy and the variables that measure individuals' risk preferences and attitude to ambiguity (the last variable is interacted with a dummy that takes value 1 in ambiguous distribution problems). We also include a dummy variable for the ability to calculate expected values (the variable takes the value 1 if the subject could correctly calculate the expected value of a simple lottery: $73 \%$ of Dictators

\footnotetext{
${ }^{6}$ Only two of the fifteen pair-wise comparisons reveal significant differences according to the Wilcoxon signed-rank test.
} 
answered correctly). ${ }^{7}$ Variables measuring individuals' beliefs about others' preferences are not included as they are highly correlated with measured preferences. We consider each treatment separately because risk preferences may differently influence dictators' generosity in the two treatments. We find that in TUR the amount D keeps for himself is positively and significantly related to D's own $\alpha$. One interpretation of this result is that dictators in TUR show some empathy for R's condition. The higher a dictator's own aversion to risk the more he is generous to $R$, who faces risk. We also observe that the amount kept by dictators in TUR increases with their ability to correctly calculate the expected value of a lottery. Generosity is thus weaker when the conditions affecting R's earnings are correctly understood. The regressions coefficients associated to the remaining explanatory variables are all insignificant. Dictators' generosity in TUD is not related to any of the considered explanatory variables. All regression results are reported in the Appendix.

Result 4.3.2. When recipients are exposed to risk, individuals' generosity increases with their aversion to risk and decreases with their ability to comprehend expected values. Individuals' generosity appears to be unaffected by observable characteristics when their own earnings are uncertain.

\subsection{Fairness norms}

In this section we present the different fairness norms that dictators may endorse when making distribution decisions. The section is based on Chapter 3 , where we theoretically and experimentally investigated fairness norms in distribution problems characterized by uncertainty. In the experiment a subject, the so called benevolent dictator (Konow, 2000), was asked to divide a certain amount of money between two equally deserving recipients, himself having no stakes in the amount to be divided. The same type of distribution problems as in this experiment were employed. In all problems, the final earnings of one of the recipients were uncertain while the

\footnotetext{
${ }^{7}$ The following, non-incentivized, question was administered at the end of the experiment: "We would like to ask you to calculate the expected value of the following lottery: with $50 \%$ chance you earn 20 Euro and with 50\% you earn 10 Euro. Please type your answer in the box below."
} 
final earnings of the other were safe. In order to understand which fairness norms motivate allocations, we first selected theories of distributive justice that can be applied to the studied distribution problems. We identified four theoretical fairness norms: ex-post equality, ex-ante equality, equity and utilitarianism. The ex-post equality fairness norm is based on the idea of minimizing outcome inequalities after uncertainty has been resolved. The implication of this norm for our experiment is that the share allocated to individuals facing earnings uncertainty should always be smaller than that of individuals whose earnings are safe. The fairness norm of ex-ante equality reflects the idea that individuals should enjoy the same utility in expectation, that is before the uncertainty affecting final earnings is resolved. More precisely, under the assumption of risk averse (seeking) preferences, the exante fairness norm prescribes to compensate individuals facing uncertain (sure) earnings. The utilitarian fairness norm delivers allocations that maximize total welfare. Hence, this norm prescribes to allocate a larger share of the total to the individual whose earnings are certain (uncertain) depending on weather the fairness norm is interpreted by risk averse (seeking) individuals. Lastly, the fairness norm inspired by equity prescribes that the total is equally split, irrespective of the uncertainty affecting a recipient's earnings. Each fairness norm produces individual-level predictions on allocations. ${ }^{8}$ We indicate with $y_{R}$ the fair allocation of the individual facing earnings' uncertainty and with $y_{C}$ the fair allocation of the individual whose earnings are certain. Table 4.3 shows the relation between $y_{R}$ and $y_{C}$ and how allocations depend on the riskiness of the distribution problem. Note that each fairness norm prescribes the equal split, $\frac{X}{2}$, in the baseline distribution problem 1-Certainty.

In Chapter 3 we estimated for each benevolent dictator (BD) which of the four fairness norms represents his allocation behavior best by minimizing the distance between predicted and observed allocations. We found that $15.5 \%$ of benevolent dictators are best represented by the ex-post and the exante fairness norm respectively, $27 \%$ are best represented by the utilitarian fairness norm and the remaining $42 \%$ by the equity norm. In the following section we estimate the distribution of fairness norm types among dictators

\footnotetext{
${ }^{8}$ The details on the analytical derivation of the fairness norms can be found in Chapter 3.
} 
Table 4.3: Fairness Norms.

\begin{tabular}{lcc}
\hline \hline & relation of $y_{R}$ to $y_{C}$ & relation of $y_{R}$ to riskiness \\
\hline ex-post equality & $y_{R}<\frac{X}{2}<y_{C}$ & non-monotonic \\
ex-ante equality & $y_{R}>\frac{X}{2}>y_{C}$ & increasing \\
utilitarian & $y_{R}<\frac{X}{2}<y_{C}$ & decreasing \\
equity & $y_{R}=\frac{X}{2}=y_{C}$ & constant \\
\hline
\end{tabular}

Note: the above relations hold assuming risk aversion.

in TUR and TUD. The distribution of fairness types in the two treatments is then compared to the one in the Benevolent Dictator game to investigate if the endorsement of a certain fairness norm depends on whether the decision maker has stakes in the distribution problem.

\subsubsection{Decision making model and estimation}

Cappelen et al. (2007) propose that a dictator is motivated by the desire to act fairly but also wants to maximize his own material payoff. We follow this approach and assume that the utility function of a dictator in a given distribution problem is characterized by: ${ }^{9}$

$$
U(y ; \cdot)=\gamma y-\frac{\beta\left(y-k^{j}\right)^{2}}{2 X}
$$

where $y$ is the amount the dictator keeps for himself, $\gamma>0$ is a parameter indicating how much importance $\mathrm{D}$ gives to material payoffs, $\beta \geq 0$ indicates how much weight is given to fairness considerations and $k^{j}$ is the fairness

\footnotetext{
${ }^{9}$ Drawing on some existing models of social preferences, Cappelen et al. (2011) have proposed two alternative formulations of the utility function in (4.1). The first, an extension of the Fehr-Schmidt model of inequity aversion (Fehr and Schmidt, 1999), delivers extreme predictions: a dictator keeps either everything or an amount exactly equal to his fair share. Given that in distribution problem 1-Certainty the allocation behavior of approximately $40 \%$ of dictators does not meet these predictions, we do not test this approach any further. The second formulation is a variation of the Bolton-Ockenfels utility function (Bolton and Ockenfels, 2000) and only differs from the one in (4.1) in that it assumes that dictators keep more the larger the total amount to be divided, $X$. Since we focus on situations where $X$ is the same in all dictator-recipient pairs, this formulation does not add to the prediction formulated above.
} 
norm endorsed by $\mathrm{D}$. The maximization of the function in (4.1) yields the following interior solution:

$$
y^{*}=k^{j}+(\gamma / \beta) X \quad(\beta \geq 0)
$$

Equation (4.2) indicates that a dictator takes at least what she considers to be the fairness norm, $k^{j}$, in the situation and possibly an additional amount that depends on how much weight she attributes to fairness concerns relative to selfish ends. A dictator characterized by a $\beta=0$ finds it optimal to always keep the entire amount $X$. For future estimation purposes, we introduce a variable $\theta: \gamma / \beta$. The more self-interested a dictator, the higher the value of $\theta$. We, therefore, refer to $\theta$ as the selfishness parameter. Introducing risk preferences in the utility function proposed in 4.1 is not straightforward. We do this by allowing $k^{j}$ to depend on the dictator's own risk attitude (see the derivation of fairness norms in Chapter 3 ) but, for tractability reasons, we assume that utility is linearly increasing in the amount kept by the dictator. ${ }^{10}$

\subsubsection{Estimates of the decision making model}

In what follows we seek to estimate, for each dictator, which of the four discussed fairness norms represents his/her allocation decisions best. Knowing the distributions of fairness norms in TUR and TUD and comparing them to the one estimated for Benevolent Dictators, we will be able to test whether dictators adopt fairness norms in a self serving way.

We assume that dictators choose allocations in order to maximize their utility but allow dictators to make mistakes in their decisions. Therefore, we estimate:

$$
y_{i n}=k_{i n}^{j}+\theta_{i} X+\epsilon_{i n}
$$

where $y_{i n}$ is the amount dictator $i$ keeps for himself in distribution problem $n, k_{i n}^{j}$ is the fair share of $i$ in problem $n$ according to the fairness norm $j$ and $\theta_{i}$ is the individual selfishness parameter. The last term in the equation

\footnotetext{
${ }^{10}$ Other formulations, for example assuming a CRRA utility function for money, would largely complicate our estimation exercise without adding much realism to the utility representation.
} 
captures the idea that dictators may make mistakes when choosing allocations, where we assume $\epsilon_{i n} \sim N\left(0, \sigma^{2}\right)$.

Recall that in distribution problem 1-Certainty $k^{j}=X / 2 \forall j$, that is each fairness norm predicts an equal split of the joint account. Hence, we can use dictators' allocations in this distribution problem to infer the value of $\theta_{i}$ at the individual level. The derivation of $\theta_{i}$ is a preliminary step in the estimation of the fairness norms' distribution, which is derived under the assumption that individuals' selfishness is constant across distribution problems. We assume that $\theta_{i}$ can be measured without error. As a matter of fact, In Chapter 3 we show that $93 \%$ of Benevolent Dictators choose precisely the equal split and this suggests that decision errors are rather uncommon in the baseline problem.

We find that the average $\theta$ is equal to 0.29 in TUR and to 0.27 in TUD (Wilcoxon rank-sum test $\mathrm{p}=0.81$ ), meaning that on average dictators take approximately $30 \%$ of the total amount on top of what they consider their fair share.

Keeping the elicited individual $\theta_{i}$ fixed, for each dictator we search for the fairness norm that minimizes the sum of the squared distances between the predicted and the observed allocations in distribution problems characterized by uncertainty. ${ }^{11}$ That is;

$$
\min _{k^{j}} \sum_{n=1}^{6}\left(y_{i n}-\theta_{i} X-k_{i n}^{j}\right)^{2}, j=1, . ., 4 .
$$

The first two columns of Table 4.4 show the estimated distribution of fairness types in the two treatments. ${ }^{12}$ In the third column we report the distribution of fairness types estimated in the Benevolent Dictator experiment.

When comparing the distribution of fairness types we find no statistically significant difference between TUR and TUD (Fischer exact test $p=0.22$ ). However, as suggested by the figures in the table, the distribution of fairness

\footnotetext{
${ }^{11}$ In order to calculate fairness norms in ambiguous distribution problems we assume that the probability of the ambiguous event is $50 \%$, consistently with the principle of insufficient reason.

1228 Dictators (15 in TUR and 13 in TUD) that keep the whole sum in the joint account in all distribution problems, including the Certainty-1, are excluded from the analysis. We interpret the behavior of these subjects as being completely self-interested: $\beta=0$ in 4.1 and thus $y^{*}=X$.
} 
Table 4.4: Fairness types

\begin{tabular}{llll} 
& TUR & TUD & BD \\
\hline ex-post & $21 \%$ & $24 \%$ & $15.5 \%$ \\
\hline ex-ante & $16 \%$ & $31 \%$ & $15.5 \%$ \\
\hline utilitarian & $10 \%$ & $6 \%$ & $27 \%$ \\
\hline equity & $53 \%$ & $39 \%$ & $42 \%$ \\
\hline $\mathrm{N}$ & 49 & 54 & 28
\end{tabular}

types differs more markedly when comparing situations where the decision maker has (TUR and TUD) or has not (BD) stakes in the amount to be divided. We find that distribution in TUR is not, and that in the TUD is, significantly different than in the $\mathrm{BD}$ experiment (Fischer exact test $\mathrm{p}=0.14$ and $p=0.01$ respectively). In the following we investigate whether these differences can be attributed to the presence in TUR and TUD of self-serving biased types. In order to do so, we first rank the fairness ideals by the extent to which they allow a dictator to keep money controlling for risk averse and risk seeking preferences. Table 4.5 shows the ranking.

Table 4.5: Self-serving ranking of fairness norms.

TUR

\begin{tabular}{lll}
\cline { 2 - 3 } 1. & risk averse & risk seeking \\
2. & ex-post & ex-antarian \\
3. & equity & equity \\
4. & ex-ante & utilitarian
\end{tabular}

TUD

\begin{tabular}{ll}
\hline risk averse & risk seeking \\
\hline ex-ante & utilitarian \\
equity & equity \\
ex-post & ex-ante \\
utilitarian & ex-post
\end{tabular}

In TUR, risk averse dictators benefit the most by adopting the utilitarian view, which allows them to keep more than the equal split in all decision situations. The least advantageous fairness ideal is ex-ante equality, which prescribes compensation of the recipient for his/her exposure to uncertainty. Risk seeking dictators can keep most money for themselves when endorsing the ex-post equality fairness ideal and the least when adopting a utilitarian 
view. In TUD earnings uncertainty lies on the dictator side. Therefore, the ranking of ideals is precisely opposite to the ranking in TUD. We proceed by comparing the frequency of each fairness types in TUR and TUD to that in the BD experiment, using the one-sided Fischer exact test. In all experiments, we separate individuals into risk averse and risk seeking by using the estimated CRRA, $\alpha$. Table 4.6 shows the results. If, compared to the BD experiment, the distribution of fairness types in TUR and TUD is biased in favor of fairness norms that favor dictators' own material interests, we would conclude that fairness views are adopted in a self-serving way.

Table 4.6: Fairness types by risk attitude.

\begin{tabular}{lllllll} 
& \multicolumn{3}{c}{ risk averse } & \multicolumn{3}{c}{ risk seeking } \\
\hline & TUR & TUD & BD & TUR & TUD & BD \\
\hline ex-post & $16.3 \%$ & $21 \%$ & $4 \%$ & $50 \%$ & $31 \%$ & $60 \%$ \\
\hline ex-ante & $16.3 \%$ & $34 \%$ & $9 \%$ & $16.7 \%$ & $25 \%$ & $40 \%$ \\
\hline utilitarian & $9.3 \%$ & $5 \%$ & $39 \%$ & $16.7 \%$ & $6 \%$ & $0 \%$ \\
\hline equity & $58 \%$ & $40 \%$ & $48 \%$ & $16.7 \%$ & $38 \%$ & $0 \%$ \\
\hline $\mathrm{N}$ & 43 & 38 & 23 & 6 & 16 & 5
\end{tabular}

For risk averse individuals, we find that the frequency of utilitarian types is significantly smaller in TUR than in the Benevolent Dictator experiment ( $\mathrm{p}=0.01$ ), while the frequency of all other types is not significantly different in the two experiments $(p \geq 0.15)$. This result is counter to the selfserving hypothesis: the endorsement of the most favorable fairness norm is less common among involved dictators than among non-involved individuals.

In TUD the frequency of utilitarian risk averse types is statistically significantly smaller, and that of ex-ante equality fairness types larger, than in the Benevolent Dictator experiment ( $p=0.001$ and $p=0.02$ respectively). Given that utilitarianism is the least, and ex-ante fairness the most, favorable fairness ideal for a risk averse dictator, these results indicate the presence of self-serving biased dictators in TUD.

Result 4.4.1. Risk averse individuals are likely to adopt a self-serving inter- 
pretation of fairness when uncertainty affects their own earnings. However, if uncertainty affects the earnings of others, individuals' endorsement of fairness norms is not biased by self-interest.

For risk seeking individuals, we do not find any significant difference between TUR, TUD and the Benevolent Dictator experiment. However, this is mainly attributable to the very limited number of risk seeking subjects in all experiments.

\subsection{Conclusions}

In this paper we have investigated individuals' generosity when its final consequences are uncertain. Our design allowed comparing situations where the decision maker himself is affected by uncertainty to situations where uncertainty only affects the recipient. Furthermore, we varied the degree of uncertainty such that distribution problems differed by the potential inequality in final earnings.

We find that on average individuals' behavior is largely independent of whether uncertainty affects their own or others' outcomes. However, when uncertainty is high, and outcomes potentially extreme, individuals tend to act much selfishly. The apparent unresponsiveness of generosity to uncertainty is called into question once we control for the fairness norms that apply to the studied distribution problems. Assuming that one's inclination to act selfishly is constant and measurable at the individual level, we were able to estimate which of four fairness norms best represents an individual's behavior across distribution problems. Since fairness norms differ in the extent to which they entitle individuals to retain money, we could test the the presence of self-serving biased dictators by comparing the distribution of fairness norms among non-involved individuals to that among dictators. We observe that the endorsement of advantageous fairness norms is significantly higher when uncertainty affects one's own earnings. On the other hand, when the earnings of the recipient are uncertain, giving does not seem to be affected by self-serving considerations.

Our results demonstrate the importance of accounting for individuals' unbiased fairness norms when analyzing generous behavior. We find that in- 
dividuals tend to exploit uncertainty in a self-serving way when they are personally affected by it. Perhaps surprisingly, individuals do not seem to exploit others' exposure to uncertainty in a self-serving way. A possible explanation relates to the fact that individuals are on average uncertainty averse: empathy with the recipient's condition may then reduce the room for biased interpretations of fairness. 


\subsection{Appendix}

\subsubsection{Tables}

Table 4.7: Euro kept by D, all distribution problems.

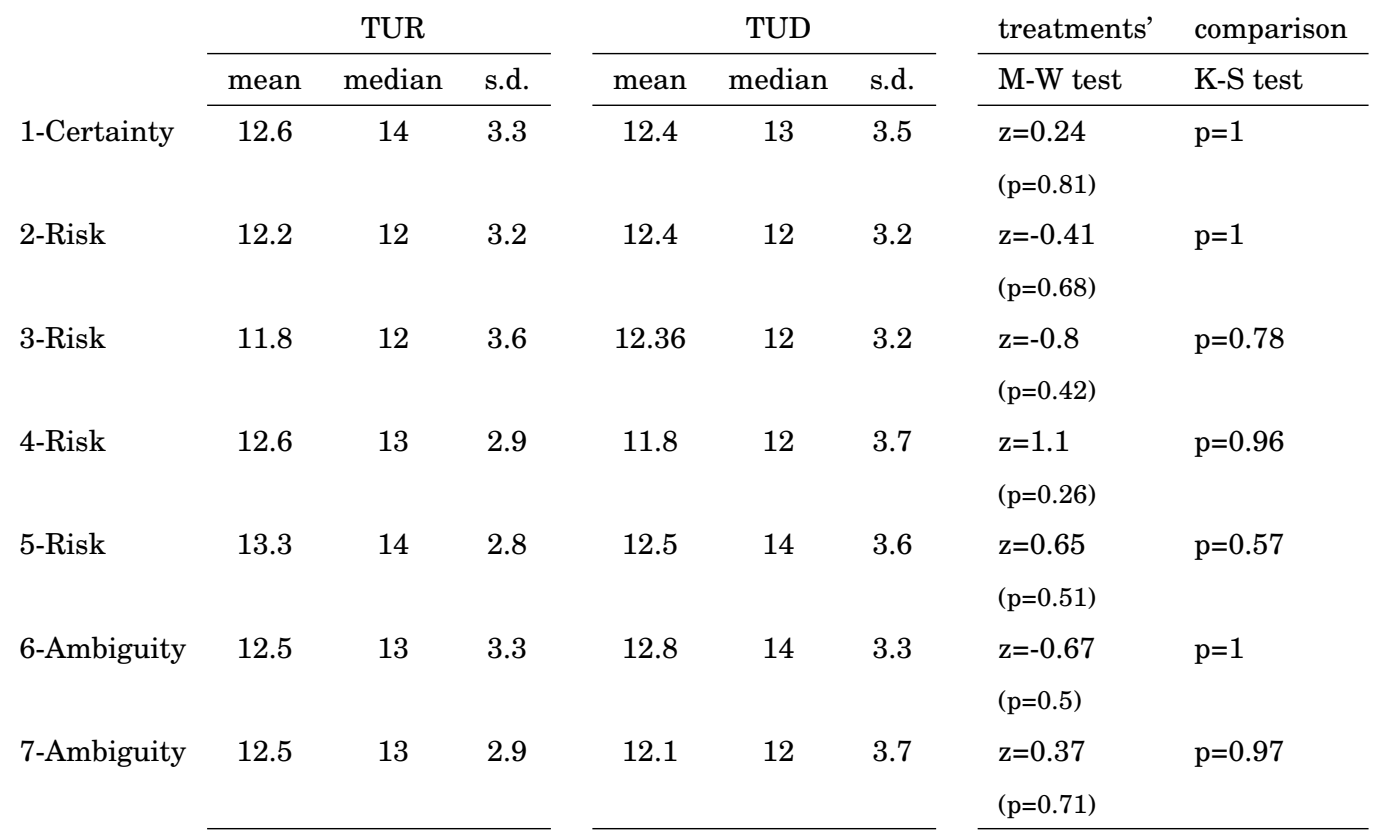

Notes. The null hypothesis of the M-W test is that the median amount kept by D is the same in both treatments. The null hypothesis in the K-S test is that the amount kept by $\mathrm{D}$ has the same distribution in both treatments. 
Table 4.8: Euro amount kept by D, tobit regression results TUR.

\begin{tabular}{lccc}
\hline \hline & \multicolumn{3}{c}{ dependent variable: Euro amount kept by D. } \\
\cline { 2 - 4 } independent variables & coefficient & standard error & p-value \\
\hline$\alpha$ & 3.37 & 1.48 & 0.02 \\
prior beliefs & -3.84 & 3.96 & 0.33 \\
female & 0.29 & 0.99 & 0.77 \\
correct EV & 2.25 & 1.03 & 0.03 \\
constant & 9.09 & 1.85 & 0.00 \\
pseudo R-squared & 0.025 & & \\
Prob>F & 0.0003 & & \\
$\mathrm{~N}$ & 448 & & \\
\hline
\end{tabular}

Distribution problem dummies included and significant.

Standard errors are robust to heteroskedasticity and are clustered on subjects.

Table 4.9: Euro amount kept by D, tobit regression results TUD.

\begin{tabular}{lccc}
\hline \hline & \multicolumn{3}{c}{ dependent variable: Euro amount kept by D. } \\
\cline { 2 - 4 } independent variables & coefficient & standard error & p-value \\
\hline$\alpha$ & -0.23 & 1.57 & 0.88 \\
prior beliefs & 3.84 & 5.29 & 0.47 \\
female & -0.63 & 0.99 & 0.52 \\
correct EV & -0.06 & 0.96 & 0.95 \\
constant & 14.06 & 1.55 & 0.00 \\
pseudo R-squared & 0.0023 & & \\
Prob>F & 0.74 & & \\
$\mathrm{~N}$ & 469 & & \\
\hline
\end{tabular}

Distribution problem dummies included and significant.

Standard errors are robust to heteroskedasticity and are clustered on subjects.

\subsubsection{Instructions of the experiment}

We report the instructions for the TUD treatment, the instructions for the TUR treatment only differ in Part 1. 


\section{Introduction speech}

In this experiment you can earn money with the decisions you make. Your earnings may also depend on chance events and the decisions of other participants. At the end of the experiment you will be paid out in cash individually and confidentially. In order to ensure the highest level of anonymity and confidentiality, the payment will be carried out by a person that is not involved in this research project. The experimenters cannot link your earnings and decisions to your identity in any way. During the experiment you are not allowed to communicate in any other way than described in the instructions. If you have any questions please raise your hand. An experimenter will then come to you and answer your questions in private. The experiment consists of 3 parts. You will receive the instructions of a part only after the previous part has ended.

\section{Part 1}

In the first part of the experiment you will be randomly matched into groups of two participants, which will be labeled with the letters A and B. The slider task In this part of the experiment every participant is asked to perform a task that involves correctly positioning sliders on a bar. Below you can see the representation of a slider in the initial position a) and in the correct position b), which is always in the middle of the bar. The slider is positioned correctly if the number that shows up to the right of the slider equals 50 .

Figure 4.5: a) initial position of the slider

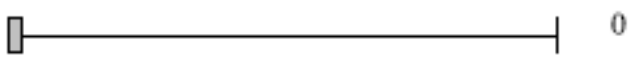

Figure 4.6: b) correct position of the slider

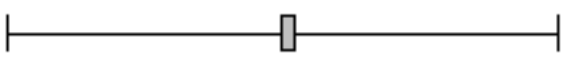

For each correctly positioned slider 0.25 Euro are credited. There are a total of 32 sliders to be positioned in 6 minutes time, so every participant 
can credit up to 8 Euro. After the 6 minutes are over, the credit accumulated by $A$ and $B$, who are in the same group, is deposited in a joint group account. Each member of a group (A and B) is then informed about how much she/he and the other member in the group contributed to the joint account. Determination of earnings The task of $\mathrm{A}$ is to divide the amount of money in the joint account between him/herself and B. A is asked to make a division in 7 different decision situations. At the end of the experiment one out of the 7 decisions will be randomly selected to determine the earnings of A and B. Each decision situation is equally likely to be the one that determines the earnings of A and B. Therefore, person A should carefully consider each decision and make each decision in isolation. The 7 decision situations differ in the way the amount of money assigned to A and B translates into earnings for $\mathrm{A}$ and $\mathrm{B}$. The table below summarizes the 7 decision situations and shows how the earnings of A and B are determined in each decision situation. Notice that during the experiment the 7 decision situations will appear in random order. Please have a look at it.

\begin{tabular}{lll}
\hline & Earnings of A & Earnings of B \\
\hline \hline 1 & allocation to A & allocation to B \\
2 & $20 \%$ chance 5 times allocation to A, $80 \%$ times 0 & allocation to B \\
3 & $50 \%$ chance 2 times allocation to A, $50 \%$ times 0 & allocation to B \\
4 & $80 \%$ chance 1.25 times allocation to A, 20\% times 0 & allocation to B \\
5 & $50 \%$ chance 1.5 times allocation to A, $50 \%$ times 0.5 times allocation to A & allocation to B \\
6 & unknown chance 2 times allocation to A, unknown chance 0 & allocation to B \\
7 & unknown chance 1.5 times allocation to A, unknown chance 0.5 times allocation to A & allocation to B \\
\hline
\end{tabular}

We will now explain each decision situation in detail. If, at the end of the experiment, decision situation 1 is selected to matter for payment then the earnings of $A$ are equal to the amount $A$ allocated to him/herself and the earnings of $\mathrm{B}$ are equal to the allocation to $\mathrm{B}$.

If, at the end of the experiment, decision situation 2 is selected to matter for payment the earnings of $\mathrm{A}$ are equal to the allocation to A. The final earnings of B depend on the amount of Euro allocated to B and on a chance event. The chance event will be the public drawing of a card from a stack 
of 100 cards numbered from 1 to 100 . If a card with a number from 1 to 20 will be drawn then the earnings of $\mathrm{B}$ will be 5 times the money allocated to B (i.e., $500 \%$ of the allocation to B). If a number from 21 to 100 will be drawn then the earnings of $B$ will be 0 Euro. In other words, with $20 \%$ chance the earnings of $\mathrm{B}$ will be 5 times the allocation to $\mathrm{B}$ and with $80 \%$ chance the earnings of $B$ will be 0 Euro.

If, at the end of the experiment, decision situation 3 is selected to matter for payment the earnings of $A$ are equal to the allocation to $A$. The final earnings of B depend on the amount of Euro allocated to B and on a chance event. The chance event will the public drawing of a card from a stack of 100 cards numbered from 1 to 100 . If a card with a number from 1 to 50 will be drawn then the earnings of $B$ will be 2 times the allocation to B (i.e. $200 \%$ of the allocation to $B$ ). If a number from 51 to 100 will be drawn then the earnings of $\mathrm{B}$ will be 0 Euro. In other words, with $50 \%$ chance the earnings of $\mathrm{B}$ will be 2 times the allocation to $\mathrm{B}$ and with $50 \%$ chance the earnings of B will be 0 Euro.

If, at the end of the experiment, decision situation 4 is selected to matter for payment the earnings of $A$ are equal to the allocation to $A$. The final earnings of $\mathrm{B}$ depend on the amount of Euro allocated to B and on a chance event. The chance event will the public drawing of a card from a stack of 100 cards numbered from 1 to 100. If a card with a number from 1 to 80 will be drawn then the earnings of $B$ will be 1.25 times the allocation to $B$ (i.e. $125 \%$ of the allocation to B). If a number from 81 to 100 will be drawn then the earnings of $B$ will be 0 Euro. In other words, with $80 \%$ chance the earnings of $B$ will be 1.25 times the allocation to $B$ and with $20 \%$ chance the earnings of $\mathrm{B}$ will be 0 Euro.

If, at the end of the experiment, decision situation 5 is selected to matter for payment the earnings of $\mathrm{A}$ are equal to the allocation to $\mathrm{A}$. The final earnings of B depend on the amount of Euro allocated to B and on a chance event. The chance event will the public drawing of a card from a stack of 100 cards numbered from 1 to 100 . If a card with a number from 1 to 50 will be drawn then the earnings of B will be 1.5 times the allocation to B (i.e. $150 \%$ of the allocation to $B$ ). If a number from 51 to 100 will be drawn then the earnings of $\mathrm{B}$ will be 0.5 times the allocation to $\mathrm{B}$ (i.e. $50 \%$ of the allocation to $\mathrm{B})$. In other words, with $50 \%$ chance the earnings of B will be 1.5 times 
the allocation to $\mathrm{B}$ and with $50 \%$ chance the earnings of $\mathrm{B}$ will be 0.5 times the allocation to $\mathrm{B}$.

If, at the end of the experiment, decision situation 6 is selected to matter for payment the earnings of $A$ are equal to the allocation to $A$. The final earnings of $\mathrm{B}$ depend on the amount of Euro allocated to $\mathrm{B}$ and on a chance event. The experimenters will first randomly select black or red to be the winning color. The chance event will then be the public drawing of a card from a stack of 100 cards which are black or red. The total number of red and black cards sums up to 100, but neither A nor B nor the experimenters know how many red cards and how many black cards are in the stack. If a card with the winning color is drawn the earnings of $\mathrm{B}$ will be 2 times the allocation to B (i.e. $200 \%$ of the allocation to B). If a card with the losing color is drawn then the earnings of $B$ will be 0 Euro. In other words, with an unknown chance the earnings of $B$ will be 2 times the allocation to $B$ and with an unknown chance the earnings of $B$ will be 0 Euro.

If, at the end of the experiment, decision situation 7 is selected to matter for payment the earnings of $\mathrm{A}$ are equal to the allocation to $\mathrm{A}$. The final earnings of B depend on the amount of Euro allocated to B and on a chance event. The experimenters will first randomly select black or red to be the winning color. The chance event will then be the public drawing of a card from a stack of 100 cards which are black or red. The total number of red and black cards sums up to 100, but neither A nor B nor the experimenters know how many red cards and how many black cards are in the stack. If a card with the winning color is drawn the earnings of $\mathrm{B}$ will be 1.5 times the allocation to B (i.e. $150 \%$ of the allocation to B). If a card with the losing color is drawn then the earnings of $\mathrm{B}$ will be 0.5 times the allocation to $\mathrm{B}$ (i.e. $50 \%$ of the allocation to B). In other words, with an unknown chance the earnings of $\mathrm{B}$ will be 1.5 times the allocation to $\mathrm{B}$ and with an unknown chance the earnings of $\mathrm{B}$ will be 0.5 times the allocation to $\mathrm{B}$.

If you have any question please raise your hand and an experimenter will come to answer your question in private. In the following you are asked a few questions that will help us assessing your understanding of the decision situations described above. Please fill in the missing figures. Note, that in these questions we are not interested in the actual numbers you fill in but only if you fill them in correctly. During the experiment you will have the 
possibility to use a calculator by clicking on the icon in the bottom right corner of the screen. When you are ready please raise your hand and an experimenter will come to you to check your answers. Once you are ready please wait quietly.

Consider decision situation 3 and assume that the total in the joint account is 16 Euro. If A assigns... Euro to him/herself and ... Euro to B, then this means that with...\% chance B earns ...Euro and with...\% chance ....Euro. A earns .... Euro.

Consider decision situation 5 and assume that the total in the joint account is 15 Euro. If A assigns ... Euro to him/herself and... Euro to B, then this means that with ...\% chance B earns ... Euro and with...\% chance ....Euro. A earns .... Euro.

Consider decision situation 7 and assume that the total in the joint account is 12 Euro. If A assigns ... Euro to him/herself and .... Euro to B, then this means that with ...\% chance B earns ... Euro and with ...\% chance ....Euro. A earns... Euro.

\section{Part 2}

You are now going to make a series of decisions. These decisions will not influence your earnings from the first part of the experiment, nor will the decisions you made in the first part of the experiment influence the earnings from this part. Furthermore, the decisions you are going to make will only influence your own earnings.

You will be confronted with 12 decision situations. All these decision situations are completely independent of each other. A choice you made in one decision situation does not affect any of the other following decision situations.

Each decision situation is displayed on a screen. The screen consists of 20 rows. You have to decide for every row whether you prefer option A or option B. Option A is the same for every row in a given decision situation, while option B takes 20 different values, one for each row. Note that within a decision situation you can only switch once from option B to option A: if you switch more than once a warning message will appear on the screen and you will be asked to change your decisions. By clicking on NEXT you will see 
some examples screens of decision situations.

This is a screen shot of a typical decision situation that you are going to face. You are not asked to make choices now! Please have a careful look. Thereafter click on NEXT to proceed.

\section{[INSERT SCREENSHOT 1 HERE]}

This is another screen shot of a typical decision situation that you are going to face. If you want to review the previous example click on BACK, otherwise click on NEXT to proceed.

\section{[INSERT SCREENSHOT 2 HERE]}

Determination of earnings At the end of the experiment one of the 12 decision situations will be randomly selected with equal probability. Once the decision situation is selected, one of the 20 rows in this decision situation will be randomly selected with equal probability. The choice you have made in this specific row will determine your earnings.

Consider, for instance, the first screen shot that you have seen. Option A gives you a $25 \%$ chance to earn 16 .- Euro and a $75 \%$ chance to earn $4 .-$ Euro. Option B is always a sure amount that ranges from 16.- Euro in the first row, to 4.60 Euro in the 20 th row. Suppose that the 12 th row is randomly selected. If you would have selected option B, you would receive 9.40 Euro. If, instead, you would have selected option A, the outcome of the lottery determines your earnings. At the end of the experiment the lottery outcome will be publicly determined by randomly drawing a card from a stack of numbered cards.

Consider now the second screen shot that you have seen. Option A gives you an unknown chance to earn 12.- Euro and an unknown chance to earn 4.Euro. Option B is always a lottery that gives you different chances to earn 12.- Euro or 4.- Euro. Suppose that the 10th row is randomly selected. If you would have selected option B, you would receive 12.- Euro with $55 \%$ chance and 4.- Euro with $45 \%$ chance. If, instead, you would have selected option A, a stack of red and black cards would be used at the end of the experiment to determine whether you earn 12.- Euro or 4.- Euro. This stack of cards will be the same that has been described in part 1: recall that the exact number of black cards and the exact number of red cards in the stack are unknown 
to you and to us as well. You would earn 12.- Euro if a card of the winning color is drawn and 4.- Euro otherwise.

Please note that each decision situation has the same likelihood to be the one that is relevant for your earnings. Therefore, you should view each decision independently and consider all your choices carefully. If you like to, you can review the examples screens once more by clicking on BACK. If you have any questions please raise your hand. When you are ready, please press the BEGIN button below.

\section{Part 3}

In the following you are asked to estimate the choices made by one of your group members in 6 decision situations of the second part of the experiment. After having made these estimates you will answer a questionnaire and then the experiment will be over.

You are going to be randomly matched to one of your group members. For a certain decision situation you are asked to indicate which is the last row where you believe your matched group member chooses option B before switching to option A. You earn 1 Euro if you correctly indicate the switching point of your matched group member in a certain decision situation. Therefore, you can earn up to 6 Euro in total. If the true switching point of your matched group member is different from the point you indicated you earn nothing.

If you do not want to indicate a single switching point you can indicate a range of values where you think the switching point of your matched group member lies. If the true switching point lies in this range of values you will earn a positive amount smaller than 1 Euro. The exact amount you earn is calculated according to a formula. The formula captures the idea that earnings are inversely related to the length of the interval you indicate. This means that the larger the interval you indicate the smaller your potential earnings are. This formula also guarantees that your earnings are maximized if you truthfully indicate your estimate. If the true switching point of your counterpart lies outside the interval you indicate you earn nothing. Please click on NEXT to view an example.

This is a screen shot of a typical screen that you are going to see. Assume, 
for instance, that you believe that your matched group member chooses option B for the last time when option B is equal to 6.- Euro. In such a case, you would type the number 6 in both boxes at the bottom of the screen.

Assume now that you believe that your matched group member may switch from option B to option A when option B takes any value between 8.- Euro and 4.50 Euro. In such a case, you would type the number 8 in the first box and the number 4.50 in the second box. Notice that you earn nothing if you type in two values that cover all possible switching points, in this case if you type in 10 and 0.50 .

If you have any question please raise your hand. Otherwise click on NEXT to proceed.

\section{[INSERT SCREENSHOT 3 HERE]}

This is another screen shot of a typical screen that you are going to face. Assume, for instance, that you believe that your matched group member chooses option B for the last time when option B gives a chance of $40 \%$ to win 12.- Euro. In such a case, you would type the number 40 in both boxes at the bottom of the screen.

Assume now that you believe that your matched group member switches from option B to option A when the winning chance of option B is between $70 \%$ and $25 \%$. In such a case, you would type the number 70 in the first box and the number 25 in the second box. Notice that you earn nothing if you type in two values that cover all possible switching points, that is if you type in 100 and 5 .

\section{[INSERT SCREENSHOT 4 HERE]}

If you have any question please raise your hand. If you want to review the previous examples once more click on BACK. Otherwise, click on BEGIN to start the third part of the experiment. 
Figure 4.7: Screenshot 1

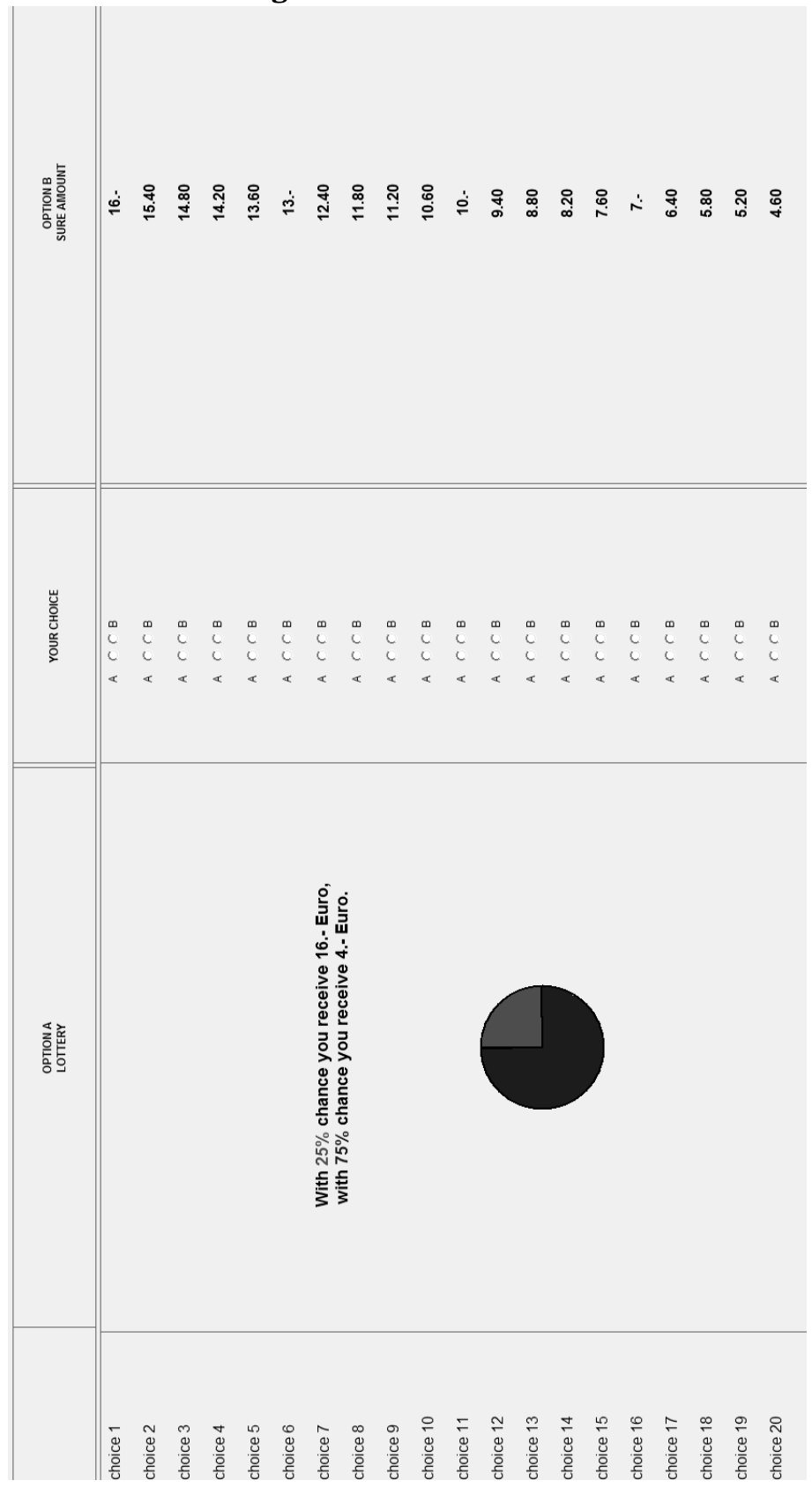


Figure 4.8: Screenshot 2

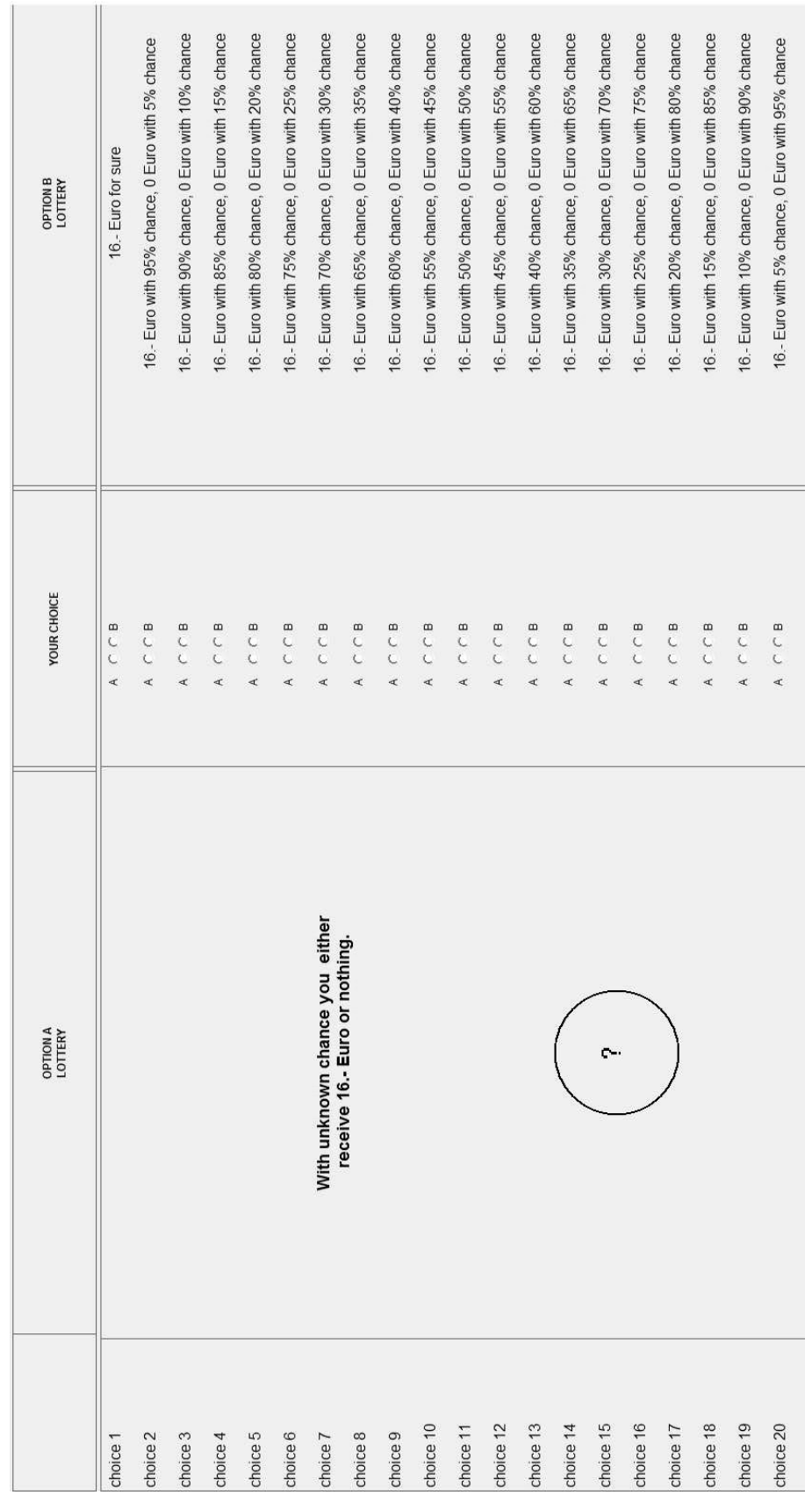


Figure 4.9: Screenshot 3

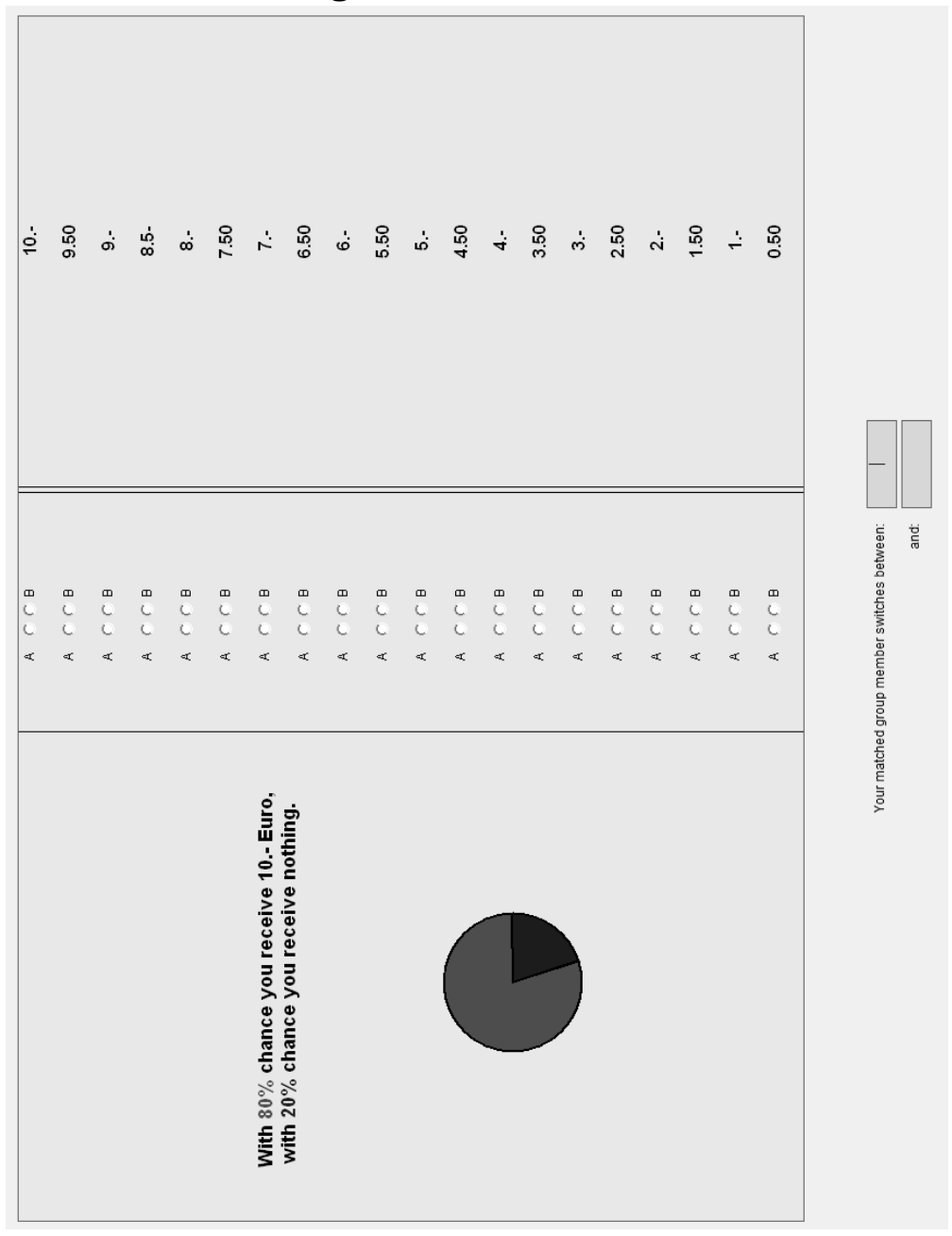


Figure 4.10: Screenshot 4

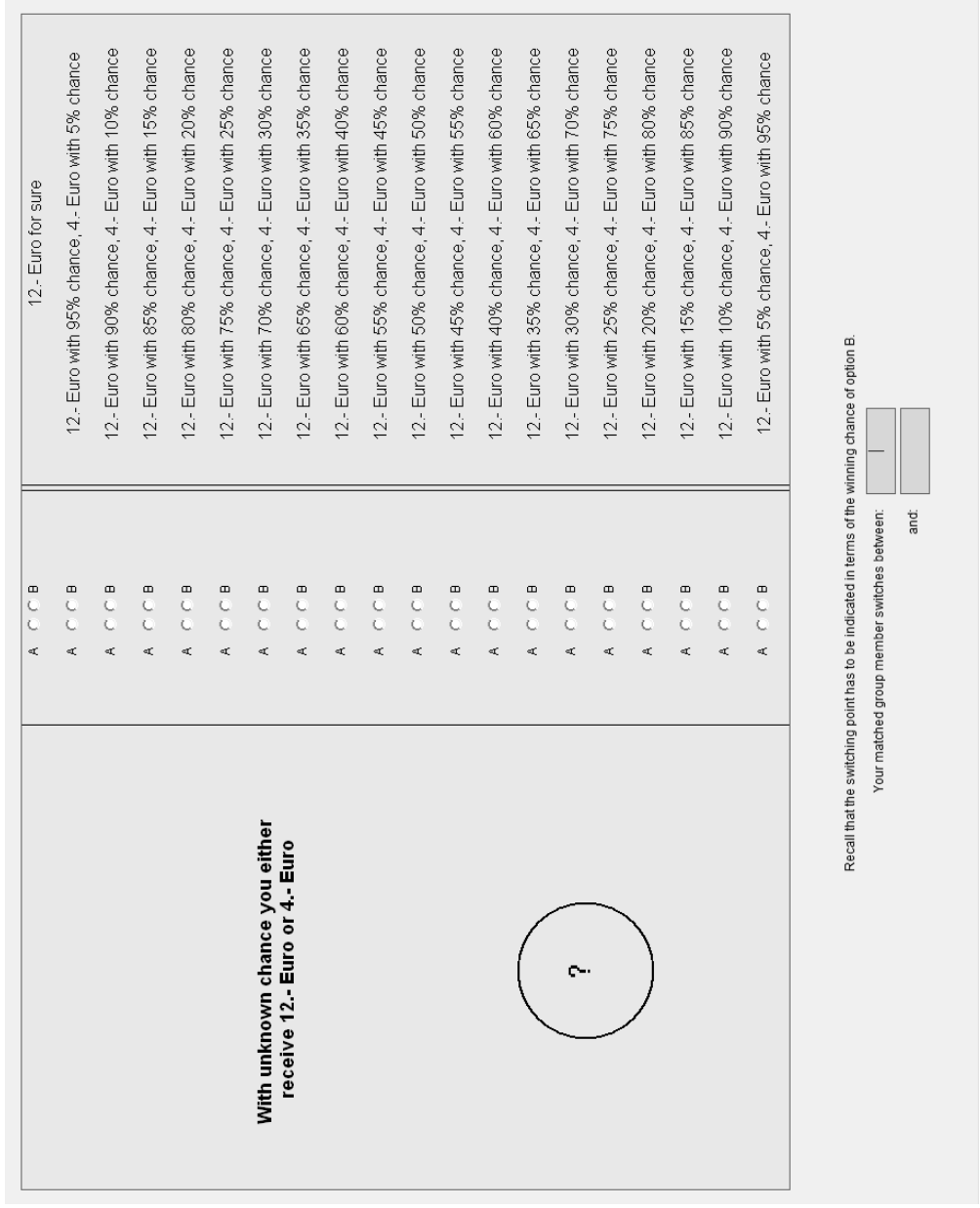





\section{Chapter 5}

\section{Risk Taking and Risk Sharing - Does \\ Accountability Matter?}

\subsection{Introduction}

Risk sharing is the essential feature of any kind of insurance system. Individual risks are pooled in order to avoid severe income losses in case an undesirable event occurs. Examples of risk sharing arrangements are the social security system that covers major risks like unemployment, disability, old age or illness, as well as voluntary sharing arrangements like fire insurance or car insurance. Crucial for the support of such an arrangement is individuals' awareness of their current risk exposure and the uncertainty regarding the event of a loss. Joining a risk sharing arrangement can be especially desirable for a risk averse individual as it allows reducing one's exposure to risks. Furthermore, the sharing of risk has redistributive consequences in the sense that individuals that are unlucky are eventually supported by those that are lucky. Thus, the more risk is shared, the more inequalities are reduced ex-post.

An important distinction has to be made between risks that cannot be influenced by individuals' behavior, such as their genetic predisposition to a certain disease, and those risks that can at least partly be influenced by their 
behavior. A high proportion of health care costs, for example, are directly caused by factors that are within patients' control like e.g., smoking, a lack of exercise, overeating, consumption of addictive substances or unsafe sex practices (see Cawley and Ruhm, 2011). Individuals exhibiting one of these habits face an increased probability of getting certain illnesses or exacerbate their consequences. Thus, they often represent an above average burden for health care providers. The awareness of this relation inspired the idea of using differential insurance rates for different risk types: the practical consequence would be that individuals with observable high risk exposure, like smokers or obese individuals, are charged a higher premium in line with their expected medical expenses. The controversial nature of this proposal is reflected in the large number of (recent) related articles in newspapers and news blogs. ${ }^{1}$ Critics argue that not all conditions are the results of fully deliberate choices but can be associated to factors beyond people's control. A recent health poll that covers more than 100.000 US households reveals that the majority of people, especially non-smokers, would favor higher health insurance payments for smokers. At the same time, however, the majority is not in favor of imposing higher insurance payments on obese individuals (see Thomas Reuters-NPR Health Poll, 2011). In sum, it seems that support for collective risk sharing is related to individuals' own risk taking behavior. Moreover, support for risk sharing seems to be related to perceived responsibility for high risk taking, as suggested by the fact that respondents' answers depend on whether higher insurance payments are targeted at smokers or obese people.

We conduct a laboratory experiment in order to systematically investigate how responsibility for risk exposure affects the support for risk sharing arrangements. Furthermore, our design allows to investigate how the support for risk sharing depends on individuals' risk preferences, their own risk exposure, as well as others' risk exposure. Understanding under which conditions people are most willing to share risks is of fundamental importance to adjust risk sharing systems in a way that their viability is ensured.

Existing experimental studies have investigated the support for income re-

\footnotetext{
${ }^{1}$ See, for instance New York Times (Nov, 2011), CNN (Oct, 2011), The Washington Post (Jan, 2012).
} 
distribution in contexts where individuals' outcomes are the product of decisions made under risk. Cappelen et al. (2010) find that impartial spectators equalize the earnings of lucky and unlucky risk takers more often than the earnings of individuals that chose a different risk exposure. In particular, willingness to reduce inequalities is lowest among individuals who chose a safe payment and are matched to an unlucky risk taker. Thral and Rademacher (2009) implement the solidarity game of Selten and Ockenfels (1998) and compare it to a treatment where individuals choose between a safe payment and a risky lottery. The authors show that individuals that chose the safe payment are less willing to reduce inequalities when matched with individuals that chose the lottery and became needy, as compared to individuals that became needy by pure chance. To summarize, it appears that high risk taking is negatively perceived by low risk takers and thus reduces their willingness to reduce inequalities. Both studies cover situations in which redistribution decisions are made at a point when individuals' outcomes are already known. However, the peculiar feature of insurance decisions is that they are taken before outcomes are realized. Hence, we test whether an accountability effect also emerges ex-ante: low risk takers may be less willing to share risks with high risk takers even if eventual outcomes are not yet known. Recent studies additionally show, that income inequalities seem to be more acceptable when they can be traced back to factors within people's control, but not if they are the result of unswayable factors. For instance, survey data reveal that support for redistribution is higher among people that think that wealth results from unjust motives, like luck or immoral behavior, as opposed to hard work, effort and skills (Alesina and Glaeser, 2004, Alesina and La Ferrara 2004, and Fong, 2011). Also experimental studies confirm the importance of the income generating process for redistribution preferences. Durante and Putterman (2009) find that subjects tend to favor less redistribution when pre-tax earnings are determined based on skill or knowledge as opposed to random assignment. Further, Krawczyk (2010) shows that individuals are more willing to reduce inequalities in situations where only luck matters as opposed to situations where the likelihood of achieving a high outcome can be affected via effort provision. In light of these results we conjecture that people's willingness to share risks may also depend on whether risk exposure is perceived to be an exogenous factor or, 
on the contrary, an individual choice variable.

The experimental set-up consists of two treatments: in the Exogenous Risks Treatment (EXO) subjects cannot influence the extent to which they are exposed to risk, while in the Endogenous Risks Treatment (ENDO) the extent of risk exposure is their own choice. In the first part of the ENDO treatment subjects face five situations where they choose one of two risky options, that have the same expected value but differ in their variance $\sigma^{2}$. The characteristics of the options are such that choices in this part should reflect individuals' risk preferences. In the first part of the EXO treatment subjects face exactly the same situations but are assigned one of the options by a random mechanism. Thereafter, subjects are paired and one subject in the pair is randomly selected to choose his/her preferred risk sharing level. Importantly, the risk sharing decision is made ex-ante, that is before the options' outcomes are determined. The risk sharing level indicates the percentage that will be subtracted from each individual's eventual option outcome. The pooled amount is then equally redistributed between the two individuals at the end of the experiment.

We implement the strategy method, which means that participants are asked to choose a risk sharing level for the case that they are matched with somebody that chose (has been assigned) the same, or the other option. Using the strategy method allows us to systematically test how people's desired level of risk sharing is related to their own risk exposure and that of the other individual in the pair. In the last part of the experiment we elicit subjects' certainty equivalents for a set of lotteries in order to estimate their CRRA (Constant Relative Risk Aversion) at the individual level.

We find that irrespective of their own risk attitude, individuals are on average less willing to share risk when their risk sharing partner has deliberately chosen a high risk option. Support for risk sharing is especially reduced among subjects who chose a low risk option instead and highest in the situations where high risk taking includes the possibility of a zero outcome, which is the lowest possible outcome among all lotteries. On the contrary, when risk exposure can not be controlled, risk sharing does not depend on the option faced by the risk sharing partner.

The reminder of the paper is organized as follows: in Section 5.2 the exper- 
imental design is described in detail. Section 5.3 summarizes theoretical predictions and hypotheses. Results are presented and discussed in section 5.4. In section 5.5 concluding remarks are drawn.

\subsection{Experimental design}

The ENDO and EXO treatments essentially differ in whether subjects can or cannot decide on their exposure to risk. Both treatments consist of three parts: in what follows we describe each part in detail and point out the treatment differences.

In the risk exposure part all subjects face five situations in which they are introduced to two options, $\mathrm{R}$ (risky) and $\mathrm{S}$ (safe). ${ }^{2}$ Both options are risky lotteries that yield a high outcome, $H$, with probability $p$ and a low outcome, $l$, with probability $1-p$. Options only differ in their variance: in all situations, the expected value is the same but the variance of option $\mathrm{S}$ is smaller than that of option R. Differently said, lottery $S$ always second order stochastically dominates lottery $\mathrm{R}$ and thus we refer to $\mathrm{R}$ as the riskier option.

In all five situations, subjects participating in the ENDO treatment are asked to make a choice between options $\mathrm{R}$ and S. On the contrary, participants in the EXO treatment are assigned one of the two options by a computer operated random draw. Options are described on the computer screen in words and with the use of an intuitive graphical display. All participants are informed that only one of the five situations will matter for their final earnings that will be determined at the end of the experiment. It was carefully explained to the subjects that each situation is equally likely to be selected for payment. Furthermore, in the ENDO treatment we specified that for this reason each choice should be made in isolation from the others.

At the beginning of the risk sharing part subjects are randomly matched in pairs and in each pair one subject is randomly selected to choose a risk sharing level $s \in[0,100]$, which represents the percentage amount that is deducted from the lottery outcome of each subject in the pair. Notice that the risk sharing level has to be chosen ex-ante, that is before the options'

\footnotetext{
${ }^{2}$ In the experiment a more neutral wording is employed. Please refer to the Appendix for the instructions used in the experiment.
} 
outcomes are known. After lotteries are resolved, the amounts deducted from the eventual individual outcomes are added at the pair level and then equally re-distributed to the two individuals. The final earnings of a subject $i$ from the first two parts of the experiment are then defined by the following expression:

$$
\Pi_{i}=\left(1-\frac{s}{100}\right) \cdot Y_{i}+\frac{\frac{s}{100} \cdot\left(Y_{i}+Y_{j}\right)}{2}, Y=H, l
$$

where $Y_{i}$ is the lottery outcome of $i$ and $Y_{j}$ is the lottery outcome of $i$ 's risk sharing partner, $j$. Note that the lottery outcomes of $i$ and $j$ are uncorrelated.

The selected subject is asked to choose a sharing level $s$ for each of the five situations in the risk exposure part of the experiment. We use the strategy method which means that for each situation two values of $s$ need to be chosen. One for the case that the other subject in the pair chose the same lottery option and one for the case that the other subject in the pair chose the alternative lottery option. At the end of the experiment subjects are informed about the actual lottery chosen by, or assigned to, their risk sharing partner. Further, the chosen risk sharing level is revealed. Risk is then resolved, the chosen redistribution is implemented and earnings are determined.

Instructions for the risk exposure and the risk sharing parts are administered together at the beginning of the experiment. Hence, in the ENDO (EXO) treatment subjects choose (are assigned) an option knowing that decisions about risk sharing will be made thereafter. This information structure was intentionally chosen to make subjects aware that their choices in the risk exposure part could influence the earnings of another participant in the experiment.

Parameters Table 5.1 gives an overview of the options employed in all five situations. Probabilities and outcomes are selected in a way that participants can easily compare the two options. In particular, option $\mathrm{R}$ and $\mathrm{S}$ are always equal in one dimension, either with respect to the outcomes' probabilities or to the value of the lower outcome of the lottery, $l$. All options have an expected value of $66 .{ }^{3}$ There are essentially two reasons for keeping

\footnotetext{
${ }^{3}$ An exception is situation II in which the expected value of option $\mathrm{S}$ is $€ 5.9$ in order to avoid confronting subjects with lottery outcomes that have more than one decimal point.
} 
the expected value of all options constant. First, within a given situation, it allows ruling out a potential explanation for risk sharing: sharing risk may be optimal for a subject in situations where the sharing partner faces a higher expected value lottery, even though this implies accepting more risk. Second, keeping expected values constant allows comparing risk sharing behavior across situations in a clean way. The order of appearance of the situations in the experiment is randomized.

Table 5.1: Situations in the risk exposure part.

\begin{tabular}{lccccc}
\hline \hline situation & option & $\mathrm{p}$ & $\mathrm{H}$ & $\mathrm{l}$ & $\sigma^{2}$ \\
\hline $\mathrm{I}$ & $\mathrm{R}$ & 0.2 & 30 & 0 & 144 \\
& $\mathrm{~S}$ & 0.5 & 12 & 0 & 36 \\
\hline II & $\mathrm{R}$ & 0.6 & 10 & 0 & 24 \\
& $\mathrm{~S}$ & 0.6 & 6.5 & 5 & 0.54 \\
\hline III & $\mathrm{R}$ & 0.2 & 22 & 2 & 64 \\
& $\mathrm{~S}$ & 0.2 & 10 & 5 & 4 \\
\hline IV & $\mathrm{R}$ & 0.2 & 14 & 4 & 16 \\
& $\mathrm{~S}$ & 0.5 & 8 & 4 & 4 \\
\hline $\mathrm{V}$ & $\mathrm{R}$ & 0.5 & 9 & 3 & 9 \\
& $\mathrm{~S}$ & 1 & 6 & 6 & 0 \\
\hline
\end{tabular}

Elicitation of risk preferences In this part of the experiment subjects' risk preferences are elicited with the multiple choice list method (Harrison and Rustrom, 2008). Participants' certainty equivalents are elicited for the first nine lotteries in Table 5.1. For each lottery subjects see a screen on the computer that contains a description of the lottery and a list of 20 equally spaced sure amounts, ranging from the lottery's highest to the lowest potential outcome. In order to facilitate comprehension, likelihoods are expressed both in percentage points and with the aid of a pie chart. In each row of the decision screen subjects have to make a choice between the lottery and the sure amount. Subjects are not allowed to switch back and forth between the sure amount and the lottery. In other words, a unique switching point is elicited for each lottery. Certainty equivalents are then calculated as the

However, as shown later, this difference does not critically affect our results. 
Chapter 5. Risk Taking and Risk Sharing - Does Accountability Matter?

arithmetic mean of the smallest sure amount preferred to the lottery and the consecutive sure amount on the list.

Experimental procedures The experiment was conducted at the Behavioral and Experimental Laboratory (BEELab) at Maastricht University. Subjects were recruited on line with the system ORSEE (Greiner, 2004). For the computerized implementation we used the experimental software Z-tree (Fischbacher, 2007). A session lasted approximately 1.5 hours and the average earnings were 18.70 Euro. In total 232 subjects participated in the experiment, 120 in the ENDO and 112 in the EXO treatment. In order to increase participants' understanding of the instructions a set of control questions was administered before the actual start of the experiment. Before being paid out and released participants are asked to fill out a questionnaire that gathers information on subjects' socio-economic characteristics.

\subsection{Theoretical predictions and hypotheses}

In the following we present the optimal risk sharing levels predicted by expected utility theory as well as by models of inequality aversion. Further, we discuss some hypotheses based on existing empirical findings.

Expected Utility Consider a subject $i$ that has been selected to choose a sharing level $s$ and is matched to a subject $j$. According to expected utility theory subject $i$ chooses $s$ in order to maximize the expected utility of his earnings:

$$
\max _{s} E U_{i}=\sum_{k=1}^{4} p_{k} \cdot U\left(\Pi_{i, k}(s)\right)
$$

where $p_{k}$ indicates the probability of the state of the world $k$, and $U(\cdot)$ is the utility of $i$ 's final earnings in state $k, \Pi_{i, k}$. The relevant states of the world are four: both subjects in the pair win, both lose, $i$ wins and $j$ loses or $j$ wins and $i$ loses. In order to make quantitative predictions on allocations, we assume that subjects are characterized by a CRRA utility function for money, that is $U(x)=x^{\alpha}$. A risk neutral individual is characterized by $\alpha=1$, a risk averse by $0<\alpha<1$ and a risk loving individual by $\alpha>1$. Recall that in the first part of the EXO treatment each subject is randomly assigned either 
option S or the riskier option R: hence, in a pair of subjects four combinations of options are possible. Furthermore, the optimal risk sharing level $s^{*}$ depends on whether a subject is risk averse or risk seeking. ${ }^{4}$ Hence, eight possible cases need to be considered: Table 5.2 summarizes the predicted optimal sharing levels for all cases. Notice that predictions are situation specific only in the case where a risk averse individual $i$ faces option $\mathrm{S}$ and individual $j$ faces option $\mathrm{R}$.

Table 5.2: Optimal sharing level $s^{*}$ of $i$.

\begin{tabular}{lcccccccc}
\hline \hline & \multicolumn{3}{c}{ i is risk averse } & \multicolumn{4}{c}{ i is risk seeking } \\
\hline i's option & $S$ & $S$ & $\mathrm{R}$ & $\mathrm{R}$ & $R$ & $R$ & $\mathrm{~S}$ & $\mathrm{~S}$ \\
j's option & $S$ & $R$ & $\mathrm{R}$ & $\mathrm{S}$ & $R$ & $S$ & $\mathrm{R}$ & $\mathrm{S}$ \\
\hline$s^{*}$ of $i$ & 100 & $10,50]$ & 100 & 100 & 0 & 0 & 100 & 0
\end{tabular}

Column three and four of Table 5.2 show that for a risk averse subject $i$ full risk sharing is optimal if assigned a risky option, irrespective of the option assigned to the other subject in the pair, $j$. This is because the benefits of reducing risk exposure predominate even if $j$ faces the riskier option $\mathrm{R}$. If $i$ is assigned option $\mathrm{S}$, the optimal sharing level is $100 \%$ if $j$ was also assigned option S. On the other hand, if $j$ is assigned option $\mathrm{R}$, the optimal sharing level is smaller than $50 \%$, but always larger than $0 .{ }^{5}$ In the latter case the optimal sharing level for $i$ results from a trade off between reducing risk exposure and facing $j$ 's riskier option R. For a risk seeking subject $i$ it is only beneficial to share risk if $j$ faces a riskier option than he does. The optimal risk sharing level in that case is $100 \%$, and 0 otherwise.

Optimal risk sharing levels are unchanged in the ENDO treatment. However, Expected Utility Theory also predicts that in the risk exposure part subjects choose an option consistent with their own risk preferences. Since option $\mathrm{S}$ second order stochastically dominates option $\mathrm{R}$ in all situations a risk averse (seeking) individual should always choose $\mathrm{S}(\mathrm{R})$. This is also

\footnotetext{
${ }^{4}$ When subject $i$ is risk neutral, there is no uniquely defined $s^{*} . E U_{i}$ is a linear combination of outcomes weighted by probabilities, and since all lotteries are characterized by the same expected value, $E U_{i}$ is constant and independent of $s$.

${ }^{5}$ The only exception is situation 5 . In this situation, if both subjects are assigned option $\mathrm{S}$, they receive a sure payment, so the optimal sharing level is not unique.
} 
true when considering that choices in the first part of the experiment can be strategically motivated. That is, subjects have no incentives to choose an option that is not in line with their own risk preferences even when they account for the existence of a successive risk sharing phase. Assume, for instance, that $i$ is risk averse: in case he will be selected to choose the risk sharing level, there is clearly no reason why he would choose the option that gives him the lower expected utility in the risk exposure part. Furthermore, even if $i$ will not be selected to decide on risk sharing, he cannot benefit from choosing the riskier option. Indeed, this will at best decrease the partner's willingness to share risks, which is always suboptimal for a risk averse subject. The relevant predictions for the ENDO treatment can be found in column 1-2 and 5-6 of Table 5.2.

Inequality Aversion Experimental research has demonstrated that, in contrast with the classical assumption of self-interested agents, the decisions of a considerable fraction of individuals are consistent with a concern for others' outcomes (Camerer, 2003). Fehr and Schmidt (1999) introduced other regarding concerns into utility analysis by assuming that an individual experiences disutility when outcomes are unequal. In particular, for a given difference in outcomes, an individual may incur a higher disutility in case he lags behind others. In our context, outcomes are not yet known when decisions about risk sharing are made. Hence, we assume that an inequality averse subject maximizes an expected Fehr-Schmidt type of utility function, where the utility derived from each possible combination of final outcomes is weighted by the outcomes' probabilities. Thus the expected utility of individual $i$ is defined as follows:

$\max _{s} E U_{i}=\sum_{k=1}^{4} p_{k} \cdot\left[\Pi_{i, k}-(1-s)\left(\alpha_{i} \cdot \max \left\{Y_{i, k}-Y_{j, k}, 0\right\}+\beta_{i} \cdot \max \left\{Y_{j, k}-Y_{i, k}, 0\right\}\right)\right]$

Where $\alpha_{i}$ measures $i$ 's aversion to disadvantageous inequality and $\beta_{i}$ measures $i$ 's aversion to advantageous inequality, with $\alpha_{i} \geq \beta_{i}$ and $0 \leq \beta_{i}<1$. The first derivative of equation (5.3) with respect to $s$ is strictly positive for all possible values of $\alpha$ and $\beta$, which implies that full risk sharing is always optimal for inequality averse individuals. Indeed, unless risks are fully shared, an individual's disutility from expected inequality is not compensated by the fact that high outcomes may only be partially shared with the 
partner. This follows from the fact that all options have the same expected value. Hence, the optimal sharing level of an inequality averse individual is $100 \%$, irrespective of his degree of inequality aversion and of the options faced by the two risk sharing partners. ${ }^{6}$

Responsibility for risk taking If subjects maximize their expected utility or a utility function that accounts for aversion to expected inequalities no difference in risk sharing is predicted between the ENDO and the EXO treatment. However, the literature reviewed in the introduction suggests that risk sharing may depend on whether risk exposure is a choice variable or exogenously given. Indeed, it has been observed that individuals are more willing to reduce inequalities in situations where only luck matters as opposed to situations where high outcomes can be achieved with effort provision (see, for instance, Krawczyk 2010). Furthermore, high risk taking seems to be negatively perceived by low risk takers, and thus reduces their willingness to reduce inequalities ex-post (see, for instance, Cappelen et al., 2010). Hence, we hypothesize that in the ENDO treatment individuals who choose a safe option may dislike a partner's voluntary high risk exposure and as a consequence share less risk with him than in the according situation in the EXO treatment.

\subsection{Results}

We start by presenting the estimates of subjects' risk preferences and proceed showing the relation of the estimates with the options chosen by subjects in the risk exposure part. This constitutes a necessary premise to the results on risk sharing behavior because, as outlined in Section 5.3, predictions on sharing levels hinge on individuals' preferences for risk and on individuals' risk exposure. We then analyze risk sharing behavior in the EXO and ENDO treatment.

\footnotetext{
${ }^{6}$ The same result holds for the inequity aversion model in Bolton and Ockenfels (2000), where individuals are assumed to incur disutility in case their outcome differs from the average outcome in the pair.
} 


\subsubsection{Risk preferences and risk exposure}

The elicited certainty equivalents in the last part of the experiment allow estimating subjects' risk preferences at the individual level. We assume a CRRA utility function for money $U(x)=x^{\alpha}$ and estimate the parameter value of $\alpha \in] 0, \infty[$ for each subject by minimizing the sum of squared distances (Wakker, 2008 and 2010). That is:

$$
\min _{\alpha} \sum_{i=1}^{9}\left[\left(p_{i} y_{i}^{\alpha}+\left(1-p_{i}\right) z_{i}^{\alpha}\right)^{\frac{1}{\alpha}}-c e_{i}\right]^{2}
$$

where the first term in brackets indicates the theoretically predicted certainty equivalent for lottery $i$, and $c e_{i}$ is the elicited certainty equivalent of lottery $i=1, . .9$. To correct for heteroscedasticity prospects are normalized to uniform length. We find that the median participant is characterized by $\alpha=0.91$, (s.d $\alpha=0.44$, mean $\alpha=0.98$ ). A majority of $62 \%$ of the participants is risk averse, a finding which is consistent with the existing literature (Holt and Laury, 2002).

In the ENDO treatment the estimated CRRA is related to the choice behavior in the risk exposure part. Indeed, we observe a positive correlation between subjects' $\alpha$ and the number of riskier options they choose (Spearman's rho $=0.48$, p-value $=0.00$ ). A large majority of $74 \%$ of risk averse subjects choose the riskier option $\mathrm{R}$ in at most two of the five situations. The choice behavior of risk seeking subjects appears to be less systematic. Still a slight majority of $57 \%$ choose option R more often than option S. Predictably, in the EXO treatment we observe no correlation between subjects' estimated risk preferences and the number of riskier options $\mathrm{R}$ assigned to them (Spearman's rho $=-0.08, \mathrm{p}=0.39$ ). This is due to the random assignment of options, which consequently creates cases where an individual is assigned an option that is not in line with his risk preferences. These cases are less frequent in the ENDO treatment, but nevertheless important to consider for our analysis of risk sharing behavior since they reveal a discrepancy between an individual's risk preferences and his choice behavior in the risk exposure part. In the following analysis we use the word (in)consistent to indicate situations where an individual chose (was assigned) an option which is (not) in line with his estimated risk preferences. 


\subsubsection{Risk sharing}

On the aggregate level, the overall average risk sharing level in the EXO treatment is not statistically significantly different from the ENDO treatment (Mann-Whitney test $\mathrm{p}=0.20$ ). Figure 5.1 shows the distributions of risk sharing decisions. In both treatments the risk sharing level for which

Figure 5.1: Distribution of risk sharing choices.

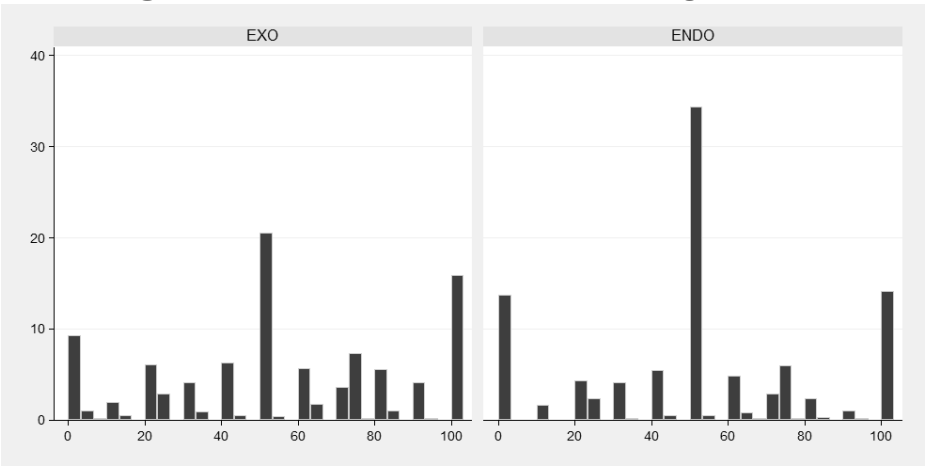

the highest frequency is observed is $50 \%$. Also, the frequency of extreme risk sharing levels of $0 \%$ and $100 \%$ is considerable. The remaining half of all risk sharing choices are intermediate values. In the following we analyze risk sharing behavior taking individuals' risk preferences, their own risk exposure and the risk exposure of their sharing partner into account. An overview of the number of observations for all cases that are covered by our analysis can be found in the Appendix.

\section{Consistent choices}

We start by analyzing the risk sharing behavior of the subjects who chose (were assigned) an option, consistent with their estimated risk preferences. Situation $\mathrm{V}$ is excluded from the analysis when we consider cases where both players in a pair face option S. Indeed, in such cases risk sharing choices are arbitrary since risk is absent.

Since we employ the strategy method in our experiment, we observe an individual's sharing behavior in both cases, where the sharing partner faces 
option $\mathrm{S}$ and option $\mathrm{R}$. In the following analysis we apply the two-tailed Wilcoxon signed-rank test for within treatment comparisons.

We first consider the behavior of consistent risk averse individuals. In the ENDO treatment subjects choose an average risk sharing level of $58 \%$ when the other member in the pair also chose option S. On the other hand, when the other member chose option $R$, the average risk sharing level is 10 percentage points lower. Statistical analysis shows that risk averse individuals who chose option S share significantly less risk if their partner chose option $\mathrm{R}$ compared to if he chose option $\mathrm{S}$ (p-value $=0.02$ ). ${ }^{7}$

Irrespective of whether they are in a pair with a subject who was assigned option S or option R, subjects in the EXO treatment choose on average sharing levels of 55\% and 53\%, respectively. Statistical tests reveal that these values are clearly not significantly different ( $p$-value $=0.86$ ).

Result 5.4.1. Consistent risk averse individuals are less willing to share risks when matched with somebody exposed to high risks only when high risk exposure is a deliberate choice.

In order to test the robustness of this result we look at risk sharing behavior in each situation separately. Figure 5.2 shows the average sharing levels chosen by risk averse individuals in the ENDO treatment. The dark blue bars display subject $i$ 's average sharing levels for the cases where both $i$ and $j$ chose option $\mathrm{S}$, whereas the light blue bars correspond to situations where $j$ instead chose option R.

We find the same patter of behavior in situations I to IV: $i$ 's willingness to share risk with $j$ is lower when $j$ chose the riskier option, $\mathrm{R}$. This difference is statistically significant in situations I and II (p-value $=0.05$ and 0.04 respectively). In these situations the low outcome associated with option $R$ is zero. In particular, in situation I choosing option $\mathrm{R}$ over option $\mathrm{S}$ goes along with an increased probability of a zero outcome. In situation II, the choice of option $\mathrm{R}$ introduces the possibility of zero earnings, whereas by choosing option S this possibility is ruled out. Differently, in situations III and IV the low outcome of both option $\mathrm{S}$ and $\mathrm{R}$ is larger than zero.

\footnotetext{
${ }^{7}$ Our unit of observation is an individual's average sharing decision calculated over those situations where the individual behavior falls into one of the four possibles combinations of options in a pair.
} 
Figure 5.2: Average risk sharing of consistent risk averse subjects (\%)- ENDO.

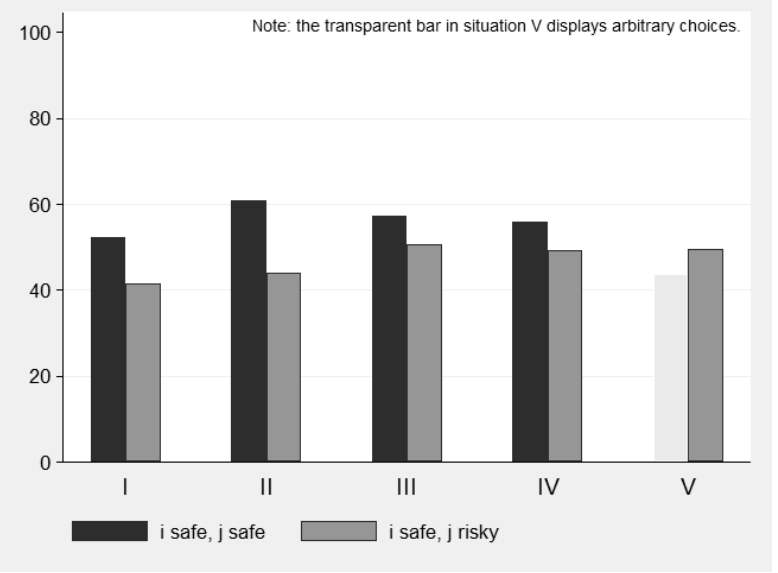

Result 5.4.2. Consistent risk averse individuals strongly differentiate their sharing behavior with respect to their partner's deliberate risk exposure if high risk taking includes the possibility of an extremely low outcome.

In the EXO treatment the situation specific tests do not reveal significant differences and we do not observe any systematic trend in behavior across situations ( $p$-value at least 0.24 ).

Given that we only find systematic differences in sharing behavior in the ENDO treatment where risk exposure is a choice variable, our results clearly suggest that accountability matters for the decision to share risk. Risk averse individuals who minimized their risk exposure in the first part of the experiment reduce risk sharing when their sharing partner refrained from doing so. Since expected utility maximization also predicts reduced risk sharing in that case, the results can also be interpreted from a different angle. Risk averse individuals might adopt a self-interested attitude only when risk exposure is endogenous, which thus implies reduced risk sharing when their partner chose a high risk that could have been avoided. However, if risks are unswayable, they choose a sharing level that leads to more ex-post equalization of outcomes, potentially backing up the sharing partner if his risk materializes in an undesirable way. Irrespective from which angle one considers the observed behavior, our results speak for the relevance of 
Chapter 5. Risk Taking and Risk Sharing - Does Accountability Matter?

perceived responsibility for risk taking behavior.

We now analyze the behavior of consistent risk seeking individuals. In the ENDO treatment individuals choose on average a sharing level of $47 \%$ in case their sharing partner also chose option R. Instead, in case the sharing partner chose option $\mathrm{S}$, the average sharing level is higher, 55\%. Statistical tests reveal that these values are not significantly different ( $p$-value $=0.23$ ). However, Figure 5.3 shows that consistent risk seeking subjects in the ENDO treatment systematically share more risks with subjects who chose the safe option. Most likely because of the limited number of observations in each situation differences are not statistically significant.

Figure 5.3: Average risk sharing of consistent risk seeking subjects (\%)- ENDO.

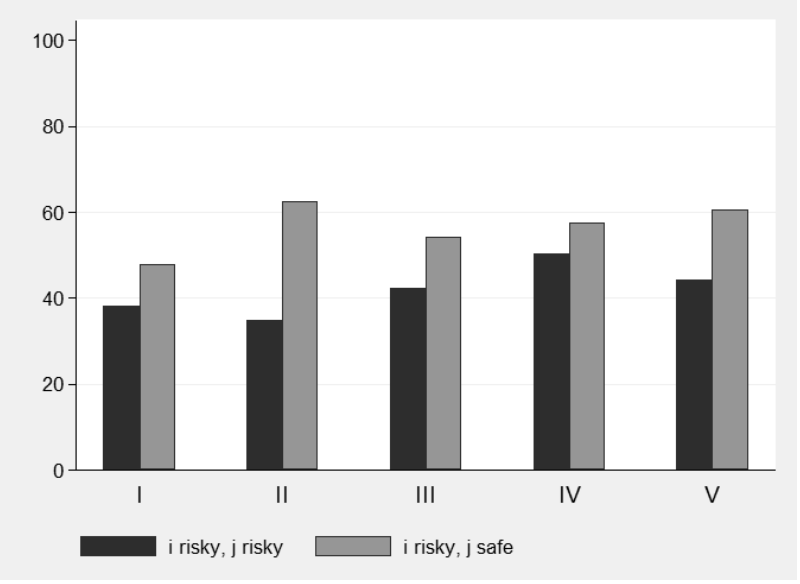

In the EXO treatment subjects choose on average sharing levels of $55 \%$ and $51 \%$ when their sharing partner was assigned option $\mathrm{R}$ and $\mathrm{S}$ respectively. This difference is clearly not statistically significant ( $p$-value=0.98). Further, we do not observe any systematic trend in behavior across situations (p-value at least 0.14).

Result 5.4.3. Individuals who deliberately and consistently chose to expose themselves to a high risk tend to share more risk with individuals that made a cautious choice. This result only holds when risk exposure is endogenous. 
The risk sharing behavior of risk seeking individuals displays the same behavioral tendency as that of risk averse individuals: facing a high risk taker as sharing partner reduces the willingness to share risks, irrespective of the own risk attitude. Therefore, it seems that not only cautious individuals condition their risk sharing on responsibility for high risk exposure, but also those that were not cautious themselves. Clearly, the lower the risk sharing level the more high risk takers have to bear the potentially bad consequences associated to option $\mathrm{R}$.

\section{Inconsistent choices}

In this section we investigate the risk sharing behavior of the individuals that chose an option that is not in line with their estimated risk preferences. We begin with analyzing the risk sharing behavior of risk averse individuals who chose (were assigned) option $R$.

In the ENDO treatment subjects chose on average a sharing level of 57\% when the other member in the pair was also assigned option R. On the other hand, when the other member was assigned option $\mathrm{S}$, the average sharing level is $49 \%$. Risk averse individuals that chose option $\mathrm{R}$ share less risk if their partner chose option $\mathrm{S}$ ( $\mathrm{p}$-value=0.11). Considering each situation separately, we find that in all situations of the ENDO treatment $i$ shares on average less risk when $j$ chose the safer option $\mathrm{S}$ as compared to when he chose R. Figure 5.4 shows the average sharing levels in situation I to V. This difference is statistically significant in situation I ( $p$-value=0.05). Differences in risk sharing levels are probably not statistically significant because only few risk averse subjects did choose option R. In the EXO treatment, average sharing levels are 58\% and 53\%: statistical tests reveal that these values are not significantly different ( $\mathrm{p}$-value $=0.37$ ). We neither observe a systematic trend nor statistically significant differences at the situation level in the EXO treatment ( $\mathrm{p}$-value at least 0.11 ).

Result 5.4.4. Risk averse individuals that are exposed to a high risk tend to share less risk when matched with somebody who chose a low risk exposure, only if risk exposure is deliberate.

In sum, Result 4 suggests that individuals who deviate from their otherwise cautious choices prefer to share more risk with individuals who made the 
Chapter 5. Risk Taking and Risk Sharing - Does Accountability Matter?

Figure 5.4: Average risk sharing of inconsistent risk averse subjects (\%) - ENDO.

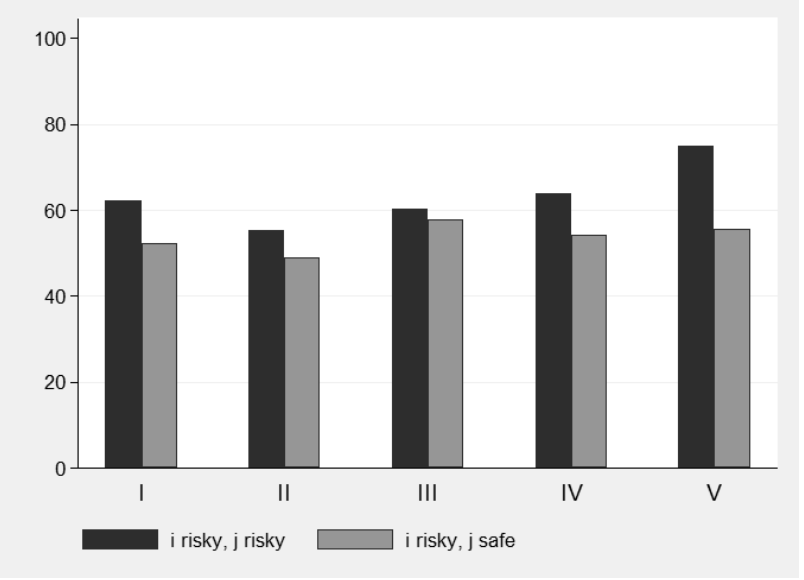

same risky choice as they did.

Lastly, we focus on the behavior of inconsistent risk seeking individuals. In the ENDO treatment subjects chose on average a sharing level of $44 \%$ when the other member in the pair was also assigned option $\mathrm{S}$. When the other member was assigned option $\mathrm{R}$, the average sharing level is $45 \%$. These sharing levels are clearly not statistically significantly different ( $\mathrm{p}$ value $=0.86$ ). The corresponding sharing levels in the EXO treatment are $57 \%$ and $62 \%$ and are also not statistically significantly different $(p$-value $=0.47)$.

Result 5.4.5. The risk sharing behavior of risk seeking individuals who chose (are exposed to) the safer option is neither significantly related to the options assigned to, nor chosen, by the other member in the pair.

The above result is confirmed when investigating risk sharing at the situation level. Indeed, in both treatments, we neither observe a systematic trend in behavior across situations nor significant differences within situations. Result 5 suggests that risk seeking individuals do not condition their risk sharing choices on the partner's risk exposure if they initially made a cautious choice. 


\subsection{Discussion and conclusions}

This study experimentally investigates how individuals' support for risk sharing is affected by deliberate versus exogenous risk exposure, by individuals' own risk preferences and by the characteristics of the risky situation. We find evidence that individual's willingness to share risks is negatively affected when other people deliberately expose themselves to high risk. The observation that low risk takers exhibit this behavior carries important practical consequences for the viability of voluntary risk sharing arrangements. Indeed, individuals who avoid habits that increase their risk margins also contribute more to the sharing pool in expectation than high risk takers. Hence their presence is fundamental for the efficient functioning of risk sharing. Our results thus suggest that the support for risk sharing arrangements by a large majority may demand measures that at least partly account for risk takers' higher expected benefits from the system. A popular measure may be, for instance, the currently debated proposal of raising smokers' health insurance premiums, which would partly shift the burden of smoking related health costs back to the smokers.

Importantly, our results can only be an underestimation of how much the support for risk sharing decreases as a consequence of high risk taking behavior. The riskier options in our set-up are characterized by potential outcomes that are more extreme in a negative and positive way as compared to their alternative counterparts. However, all options are equivalent in expectation. Thus, sharing risks with a high risk taker can be attractive, since it allows to potentially profit from a high outcome. However, habits like smoking, overeating and reckless driving have hardly positive externalities for society. Taking a high risk can at best only increase the utility of the risk taker and carry no negative consequences for the group. Hence, the observation that even in our experimental set-up less risk is shared with high risk takers can be considered a generalizable result.

An interesting observation in our experiment is that even high risk takers prefer to share more risks with those who chose a lower risk exposure. This evidence suggests that responsibility for risk exposure plays an important role for sharing behavior even for those subjects that voluntarily expose themselves to high risks. The comparison of treatments highlights a crucial 
aspect of risk sharing: people's willingness to share risks is systematically related to whether risk exposure is perceived to be a deliberate choice. Thus, our results may offer an explanation why the demand for counter balancing measures is higher for some health damaging habits than for others. Different than for smokers, many individuals might not judge obese people responsible for their preference to overeat. It remains an open question why some preferences are perceived as more legitimate, or less deserving to be condemned, than others (Bossert and Fleurbaey, 1996).

We also observe that individuals who take a high risk, but are generally cautious, are more willing to share risk with those subjects who also took a high risk. It might be that in situations where normally risk averse individuals 'dare' to take risks, they identify with other high risk takers, and thus share risks with them. This interpretation is suggested by studies on the relationship between social identification and preferences over redistribution: the more individuals identify with others the more willing they are to redistribute income ex-post (see Klor and Shayo, 2010 and Fowler and Kam, 2007). Further investigations are however needed to test the validity of this interpretation.

\subsection{Appendix}

\subsubsection{Instructions of the experiment}

The following instructions appeared on participants' computer screens (except from the headlines in squared brackets).

Part 1 [Risk exposure part]

This is a screen shot of a typical decision situation that you are going to face. You are not asked to make choices now! Please have a careful look.

In this example, Option A yields 30 Euro with $20 \%$ chance and 0 Euro with $80 \%$ chance. Option B yields 12 Euro with $50 \%$ chance and 0 Euro with $50 \%$ chance. In order to choose between Option A and Option B you will have to tick one of the boxes surrounded by the red frame.

Assume, for instance, that the decision situation above is selected to be relevant for your payment and also assume that you chose Option B. It follows 


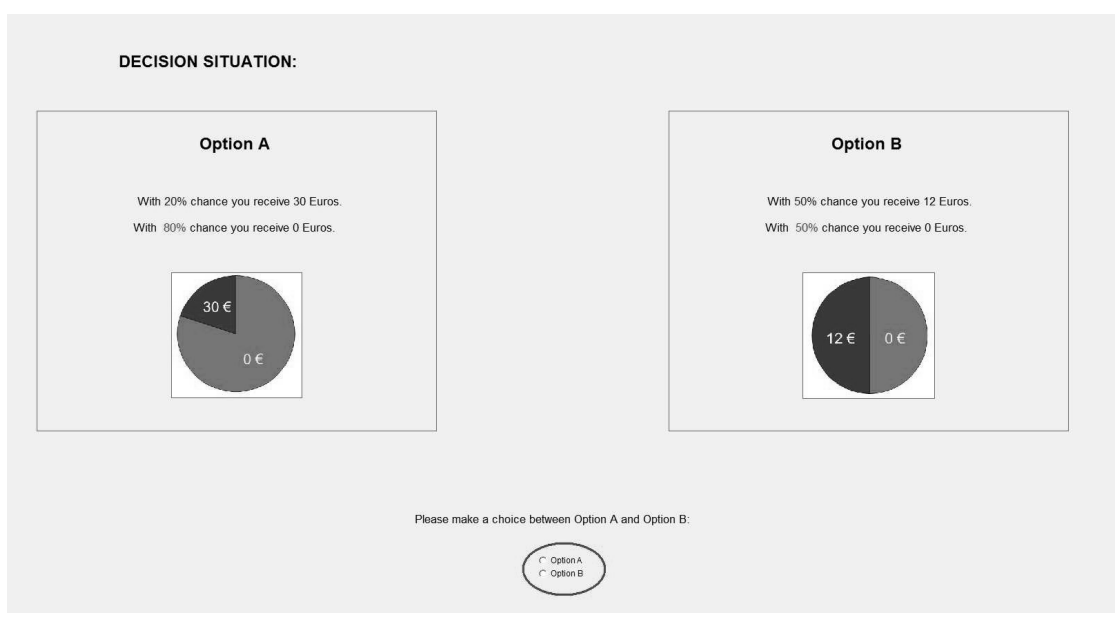

that at the end of the experiment a random draw will determine whether your outcome is 12 Euro or 0 Euro.

This outcome, together with the other decisions that you will make in this part of the experiment will determine your final earnings. In the following screens we will explain in detail how your earnings are affected by this outcome.

\section{Part 1 (continued) [Risk sharing part]}

After everyone has made choices between Option A and Option B, you will be randomly matched with another participant in the room. The two of you form a group. One person in the group (you or the other participant) will be randomly selected. For each of the 5 decision situations you faced before, the selected person has to choose a number between 1 and 100, which determines the individual outcome's percentage that each group member deposits in a group account.

At the end of the experiment, one decision situation will be randomly selected and the outcomes of the chosen options will be determined. Consequently, the amount in the group account will be calculated and equally divided between the two persons in the group. Notice that the selected person is asked to choose the percentage before the outcomes of the chosen options are known.

Assume, for instance, that you are selected to choose the percentages. In 
a given decision situation, you will have to make a choice before you know the outcome of the option you chose and before you know the outcome of the option chosen by the other person in the group.

Also notice that the selected person has to choose percentages for each of the 5 decision situations because the decision situation relevant for payment is only determined at the end of the experiment. Therefore, each choice has to be considered in isolation from the others, as if it were the one which is relevant for payment.

On the following screen the determination of earnings is illustrated with the help of examples.

\section{Part 1 (continued)}

Imagine now that you have been selected to choose the percentage.

Example 1. Assume that both you and the other person in the group have chosen Option A, which in the preceding example yields 30 Euro with $20 \%$ chance and 0 Euro with $80 \%$ chance. Let's say that you choose the percentage value $40 \%$. At the end of the experiment the uncertainty concerning your earnings is resolved; assume that Option A eventually yields 30 Euro to you and 0 Euro to the other person. It follows that:

- The group account consists of 12 Euro (that is, $0.4^{*} 30+0.4^{*} 0=12+0$ ).

- Your return from the group account is $6(=12 / 2)$ Euro.

- Your earnings are 24 Euro $(=30-12+6)$.

- The earnings of the other person in the group are 6 Euro $(=0-0+6)$.

Example 2. Assume now that in the same decision situation you have chosen Option A, which yields 30 Euro with $20 \%$ chance and 0 Euro with $80 \%$ chance, and that the other person chose Option B, which yields 12 Euro with $50 \%$ chance and 0 Euro with $50 \%$ chance. Let's say that you choose the percentage value $70 \%$. At the end of the experiment the uncertainty concerning your earnings is resolved. If Option A eventually yields 30 Euro to you and Option B yields 12 Euro to the other person. It follows that:

- The group account consists of 29.4 Euro (that is, $0.7 * 30+0.7 * 12=21+8.4$ ).

- Your return from the group account is $14.7(=29.4 / 2)$ Euro.

- Your earnings are 23.7 Euro $(=30-21+14.7)$.

- The earnings of the other person in the group are 18.3 Euro $(=12-8.4+14.7)$.

This is a screen shot of a typical decision situation that you are going to face. 
You are not asked to make choices now! Please have a careful look.

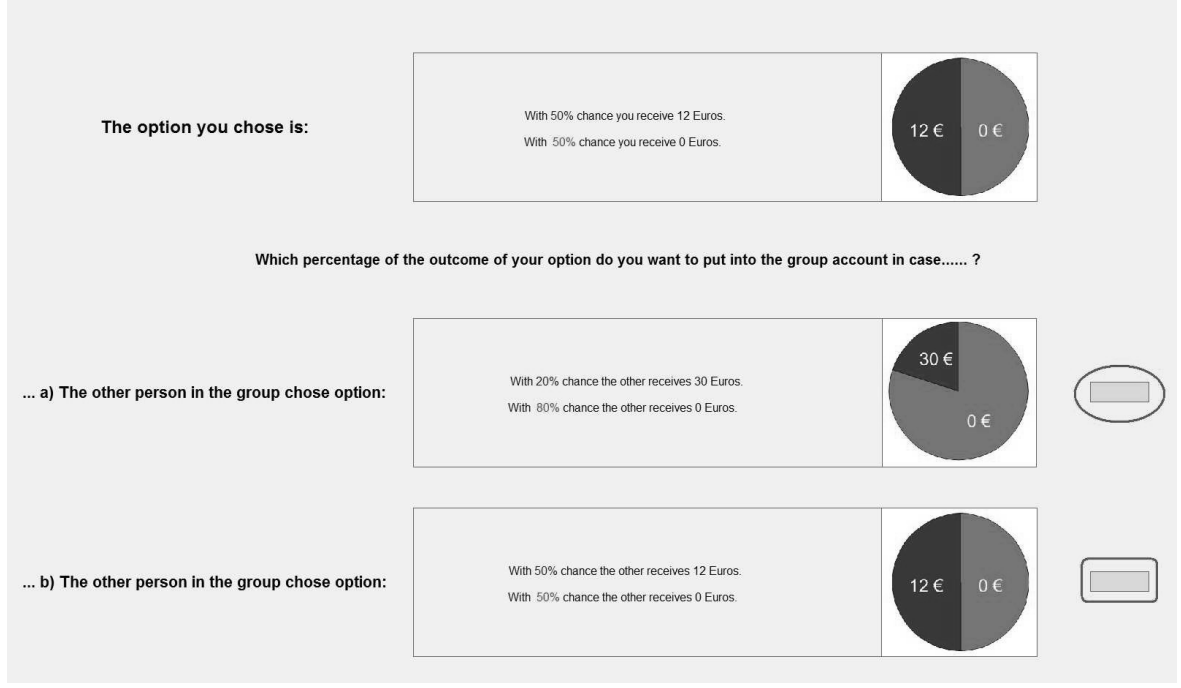

In case you are selected to choose the percentages, you are asked to do this twice for each decision situation. First, for the case that the other person in your group chose Option A (red circle) and second for the case that the other person in your group chose Option B (red square). When choosing the percentages you will not be informed about the actual option chosen by the other person in your group. At the end of the experiment the percentage associated to the actual choice of the other person in your group will be implemented. In other words, you will choose percentages for two possible scenarios. Since you do not know which one will be relevant for your payment, you have to make each choice in isolation and with the same accuracy. Notice that your outcome may be different from that of the other person in your group even if both chose the same option.

Also notice that if you choose a percentage of 0 your earnings and the earnings of the other person in the group will exclusively depend on the individual outcome of the option that each of you chose. Conversely, if you choose a percentage of 100 your earnings and the earnings of the other person in the group will be equal to each other, as they will be the sum of your outcomes divided by 2 . 


\section{Part 2 [Elicitation of risk preferences]}

You are now going to make a series of decisions. These decisions will not influence your earnings from the first part of the experiment, nor will the decisions you made in the first parts of the experiment influence the earnings from this part. Furthermore, the decisions you are going to make will only influence your own earnings.

You will be confronted with 9 decision situations. All these decision situations are completely independent of each other. Each decision situation is displayed on a screen. The screen consists of 20 rows. You have to decide for every row whether you prefer Option A or Option B. Option A is the same for every row in a given decision situation, while Option $B$ takes 20 different values, one for each row.

Note that within a decision screen you can only switch once from Option B to Option A: if you switch more than once a warning message will appear on the screen and you will be asked to change your decisions.

This is a screen shot of a typical decision situation that you are going to face. You are not asked to make choices now! Please have a careful look.

\begin{tabular}{|c|c|c|c|}
\hline & $\begin{array}{l}\text { OPTIONA } \\
\text { LOTIERY }\end{array}$ & YOUR CHOICE & $\begin{array}{l}\text { OPTION B } \\
\text { SURE AMOUNT }\end{array}$ \\
\hline choice 1 & & $A \subset \subset B$ & 12.- \\
\hline choice 2 & & $A \subset \subset B$ & 11.4 \\
\hline choice 3 & & $A \subset \subset B$ & 10.8 \\
\hline choice 4 & & $A \subset C B$ & 10.2 \\
\hline choice 5 & & $A \subset C B$ & 9.6 \\
\hline choice 6 & & $A \subset C B$ & 9.- \\
\hline choice 7 & With $50 \%$ chance you receive 12 Euros & $A \subset \subset B$ & 8.4 \\
\hline choice 8 & & $A \subset C B$ & 7.8 \\
\hline choice 9 & With $50 \%$ chance you recerve u Euros. & $A \subset \subset B$ & 7.2 \\
\hline choice 10 & & $A \subset C B$ & 6.6 \\
\hline choice 11 & & $A \subset C B$ & 6.- \\
\hline choice 12 & & $A \subset C B$ & 5.4 \\
\hline choice 13 & & $A \subset C B$ & 4.8 \\
\hline choice 14 & & $A \subset C B$ & 4.2 \\
\hline choice 15 & & $A \subset C B$ & 3.6 \\
\hline choice 16 & $12 €$ & $A \subset \subset B$ & 3.- \\
\hline choice 17 & & $A \subset C B$ & 2.4 \\
\hline choice 18 & & $A \subset C B$ & 1.8 \\
\hline choice 19 & & $A \subset C B$ & 1.2 \\
\hline choice 20 & & $A \subset C B$ & 0.6 \\
\hline
\end{tabular}

\section{Determination of earnings}

At the end of the experiment one of the 9 decision situations will be randomly 
selected with equal probability. Once the decision situation is selected, one of the 20 rows in this decision situation will be randomly selected. The choice you have made in this specific row will determine your earnings.

Consider, for instance, the first screen shot that you have seen. Option A gives you a $50 \%$ chance to earn 12 .- Euro and a $50 \%$ chance to earn nothing. Option B is always a sure amount that ranges from 12.- Euro in the first row, to 0.6 Euro in the 20th row. Suppose that the 12th row is randomly selected. If you would have selected Option B, you would receive 5.4 Euro. If, instead, you would have selected option A, the outcome of the lottery determines your earnings. At the end of the experiment the lottery outcome will be determined by the computer.

Please note that each decision situation has the same likelihood to be the one that is relevant for your earnings. Therefore, you should view each decision independently and consider all your choices carefully. 
Chapter 5. Risk Taking and Risk Sharing - Does Accountability Matter?

\subsubsection{Number of observations}

Table 5.3: EXO treatment-Choices in Risk Exposure Part.

Participant i

Participant j

\begin{tabular}{lcccccccc}
\hline & Risk averse & \multicolumn{2}{c}{ Risk seeking } & \multicolumn{2}{c}{ Risk averse } & \multicolumn{2}{c}{ Risk seeking } \\
\hline Chosen option & S & R & S & R & S & R & S & S \\
\hline Situation 1 & 22 & 18 & 8 & 8 & 16 & 14 & 18 & 8 \\
Situation 2 & 19 & 21 & 8 & 8 & 11 & 19 & 10 & 16 \\
Situation 3 & 16 & 24 & 7 & 9 & 18 & 12 & 14 & 12 \\
Situation 4 & 16 & 24 & 5 & 11 & 17 & 13 & 17 & 9 \\
Situation 5 & 21 & 19 & 8 & 8 & 10 & 20 & 11 & 15 \\
\hline
\end{tabular}

Table 5.4: ENDO treatment-Choices in Risk Exposure Part.

Participant i Participant j

\begin{tabular}{lccccccccc}
\hline & \multicolumn{1}{c}{ Risk averse } & \multicolumn{2}{c}{ Risk seeking } & \multicolumn{2}{c}{ Risk averse } & \multicolumn{2}{c}{ Risk seeking } \\
\hline Chosen option & $\mathrm{S}$ & $\mathrm{R}$ & $\mathrm{S}$ & $\mathrm{R}$ & $\mathrm{S}$ & $\mathrm{R}$ & $\mathrm{S}$ & $\mathrm{R}$ \\
\hline Situation 1 & 22 & 14 & 13 & 11 & 25 & 12 & 9 & 14 \\
Situation 2 & 22 & 14 & 16 & 8 & 26 & 11 & 14 & 9 \\
Situation 3 & 22 & 14 & 6 & 18 & 22 & 15 & 10 & 13 \\
Situation 4 & 19 & 17 & 8 & 16 & 19 & 18 & 5 & 18 \\
Situation 5 & 28 & 8 & 16 & 8 & 31 & 6 & 13 & 10 \\
\hline
\end{tabular}




\section{Bibliography}

Abdellaoui, M. (2000). Parameter-Free Elicitation of Utility and Probability Weighting Functions. Management Science, 46(11):1497-1512.

Abelson, R. The smokers' charge. New York Times on the Web, 17 November 2011.

Aguiar, F., Becker, A., Miller, L. (2010). Whose Impartiality? An experimental study of veiled stakeholders, impartial spectators and ideal observers. Jena Economic Research paper 2010-040.

Alesina, A., Glaeser, E. L. (2004). Fighting Poverty in the USA and Europe: a world of difference. Oxford University Press, Oxford.

Alesina, A., La Ferrara, E., (2005). Preferences for redistribution in the land of opportunities. Journal of Public Economics, 89:897-931.

Andreoni, J., Bernheim, D. B. (2009). Social Image and the 50-50 Norm: A Theoretical and Experimental Analysis of Audience Effects. Econometrica, 77(5):1607-1636.

Andrews, M. Smokers, forced to pay more for health insurance, can get help with quitting. The Washington Post, 2 January 2012.

Aumann, R. (1962). Utility theory without the completeness axiom. Econometrica, 30:445-462. 
Babcock, L., Loewenstein, G. (1997). Explaining Bargaining Impasse: The Role of Self-Serving Biases. Journal of Economic Perspectives, 11(1):109126.

Babcock, L., Xianghong, W., Loewenstein, G. (1996). Choosing the Wrong Pond: Social Comparisons in Negotiations That Reflect a Self-Serving Bias. The Quarterly Journal of Economics, 111(1):1-19.

Bell, D. (1982). Regret in Decision Making under Uncertainty. Operations Research, 30(5):961-981.

Benjamini, Y., Hochberg, Y. (1995). Controlling the False Discovery Rate: A Practical and Powerful Approach to Multiple Testing. Journal of the Royal Statistical Society, 57(1):289-300.

Bentham, J. (1789). An Introduction to the Principles of Morals and Legislation. London: Payne.

Bewley, T. F. (2002). Knightian decision theory. Part I. Decisions in Economic and Finance, 25:79-110.

Bolton, G.E., Ockenfels, A. (2000). A theory of equity, reciprocity and competition. American Economic Review, 100:166-193.

Bossert, W., Fleurbaey, M. (1996). Redistribution and Compensation. Social Choice and Welfare, 13(3):342-355.

Broberg, T., Ellingsen, T., Johanneson, M. (2007). Is Generosity Involuntary? Economics Letters, 94:32-37.

Cafferty, J. Should smokers and fat people pay more for health care? CNN on the Web, 31 October 2011.

Camerer, C. F. (2003). Behavioral game theory: experiments in strategic interaction. Princeton: Princeton University Press.

Camerer, C. F., Weber, M. (1992). Recent Developments in Modeling Preferences: Uncertainty and Ambiguity. Journal of Risk and Uncertainty, 5:325370. 
Cappelen, A.W., Hole A. D., Sørensen E. Ø., Tungodden, B. (2007). The Pluralism of Fairness Ideals: An Experimental Approach. American Economic Review, 97(3):818-827.

Cappelen, A.W., Konow, J., Sørensen, E. Ø., Tungodden, B. (2011). Just Luck: An Experimental Study of Risk Taking and Fairness. NHH Dept. of Economics Discussion Paper No. 4/2010.

Childress, J. F. (2001). Putting Patients First in Organ Allocation: An Ethical Analysis of the U.S. Debate. Cambridge Quarterly of Healthcare Ethics, 10(4):365-376.

Cawley, J., Ruhm, C. (2011). The economics of risky health behaviors. NBER Working Paper 17081.

Cohen, M., Jaffray, J. Y., Said, T. (1987). Experimental Comparison of Individual Behavior under Risk and under Uncertainty for Gains and for Losses. Organizational Behavior and Human Decision Processes, 39:1-22.

Dana, J., Cain, D. M., Dawes, R. M. (2006). What you don't know won't hurt me: Costly (but quiet) exit in dictator game. Organizational Behavior and Human Decision Processes, 100:193-201.

Dana, J., Weber, R., Xi Kuang, J. (2007). Exploiting moral wiggle room: experiments demonstrating an illusory preference for fairness. Economic Theory, 33:67-80.

Deutsch, M. (1985). Distributive Justice. Binghamton, NY: Vail-Ballou Press.

Dickinson, D., Tiefenhalter, J. (2002). What Is Fair? Experimental Evidence. Southern Economic Journal, 69(2):414-428.

Dohmen, T., Falk, A., Huffman, D., Sunde, U. (2010). Are Risk Aversion and Impatience Related to Cognitive Ability? American Economic Review, 100(3):1238-1260.

Dubra, J., Maccheroni, F., Ok, E. A. (2004). Expected Utility Theory without the Completeness Axiom. Journal of Economic Theory, 115:118-133. 
Durante, R., Putterman, L. (2009). Preferences for Redistribution and Perception of Fairness: An experimental Study. Working Paper.

Eliaz, K., Ok, E. (2006). Indifference or indecisiveness? Choice theoretic foundations of incomplete preferences. Games and Economic Behavior, 56: 61-86.

Ellsberg, D. (1961). Risk, Ambiguity, and the Savage Axiom. The Quarterly Journal of Economics, 75(4):643-669.

Epstein, S., Pacini, R., Denes-Raj, V., Heier, H. (1996). Individual Differences in Intuitive-Experiential and Analytical-Rational Thinking Styles. Journal of Personality and Social Psychology, 71(2): 390-405.

Faravelli, M. (2007). How Context Matters: A Survey Based Experiment on Distributive Justice. Journal of Public Economics, 91(7-8):1399-1422.

Fehr-Duda, H., Di Gennaro, M., Schubert, R. (2006). Gender, Financial Risk, and Probability Weights. Theory and Decision, 60:283-313.

Fehr, E., Schmidt, K.M. (1999). A theory of fairness, competition and cooperation. Quarterly Journal of Economics, 114:817-868.

Festinger, L. (1957). A Theory of Cognitive Dissonance. Evanston, IL: Row Peterson.

Fischbacher, U. (2007). Z-Tree: Zurich Toolbox for Ready-made Economic experiments. Experimental Economics, 10(2):171-178.

Fong, C. (2011). Social preferences, self-interest and the demand for redistribution. Journal of Public Economics, 822:225-246.

Fowler, J. H., Kam, C.D. (2007). Beyond the Self: Social Identity, Altruism, and Political Participation. Journal of Politics, 69(3):811-825.

Fox, C. R., Tversky, A. (1995). Ambiguity Aversion and Comparative Ignorance. The Quarterly Journal of Economics, 110(3):585-603.

Fox, C., Weber, M. (2002), Ambiguity Aversion, Comparative Ignorance, and Decision Context. Organizational Behavior and Human Decision Processes, 88:476-498. 
Frederick, S. (2005). Cognitive Reflection and Decision Making. Journal of Economic Perspectives, 19(4):25-42.

Gächter, S., Riedl, A. (2005). Moral Property Rights in Bargaining with Infeasible Claims. Management Science, 51(2):249-263.

Gächter, S., Riedl, A. (2006). Dividing Justly in Bargaining Problems. Social Choice and Welfare, 27:571-594.

Ghirardato, P., Maccheroni. F., Marinacci, M. (2004). Differentiating ambiguity and ambiguity attitude. Journal of Economic Theory, 118:133-173.

Gilboa, I., Maccheroni, F., Marinacci, M., Schmeidler, D. (2010). Objective and Subjective Rationality in a Multiple Prior Model. Econometrica, 78(2):755-770.

Gilboa, I., Schmeidler, D. (1989). Maxmin Expected Utility With Non Unique Prior. Journal of Mathematical Economics, 18:141-153.

Gill, D., Prowse, V. (2011). A Structural Analysis of Disappointment Aversion in a Real Effort Competition. American Economic Review, forthcoming.

Greiner, B. (2004). An Online Recruitment System for Economic Experiments. In: Kurt Kremer, Volker Macho (Hrsg.): Forschung und wissenschaftliches Rechnen. GWDG Bericht 63. Ges. für Wiss. Datenverarbeitung, Göttingen, 79-93.

Haisley, E. C., Weber, R. (2010). Self-serving interpretations of ambiguity in other-regrading behaviour. Games and Economic Behavior, 68: 614-625.

Harrison, G. W., Rustrom, E., (2008). Risk Aversion in Experiments. Research in Experimental Economics, Volume 12. J.C. Cox and G.W. Harrison (eds.).

Holt, C., Laury, S. (2002). Risk Aversion and Incentive Effects. American Economic Review, 92(5):1644-1655.

Kahneman, D., Knetsch, J.L., Thaler, R. (1986). Fairness as a Constraint on Profit Seeking: Entitlements in the Market. The American Economic Review, 76(4):728-741. 
Klor, E. F. , Shayo, M. (2010). Social identity and preferences over redistribution. Journal of Public Economics, 94(3-4):269-278.

Konow, J. (1996). A Positive Theory of Economic Fairness. Journal of Economic Behavior and Organization, 31(1):13-35.

Konow, J. (2000). Fair Shares: Accountability and Cognitive Dissonance in Allocation Decisions. American Economic Review, 90(4):1072-1091.

Konow, J. (2003). Which Is the Fairest One of All? A Positive Analysis of Justice Theories. Journal of Economic Literature, 41:1188-1239.

Krawczyk, M. (2010). A glimpse through the veil of ignorance: Equality of opportunity and support for redistribution? Journal of Public Economics, 94:131-141.

Krupka, E. L., Weber, R. A. (2008). Identifying Social Norms Using Coordination Games: Why Does Dictator Game Sharing Vary? IZA Discussion paper n. 3860 .

Lerner, J., Tetlock, P. E. (1999). Accounting for the Effects of Accountability. Psychological Bulletin, 125(2):255-275.

Loomes, G., Sugden, R. (1982). Regret Theory: An Alternative Theory of Rational Choice Under Uncertainty. The Economic Journal, 92:805-824.

Mandler, M. (2004). Status quo maintenance reconsidered: changing or incomplete preferences. The Economic Journal, 114:518-535.

Marx, K., Engels, F. (1958). Selected Works, London: Lawrence and Wishart.

Masatlioglu, Y., Ok, E. A., 2005. Rational choice with status quo bias. Journal of Economic Theory, 121(1):1-29.

Mas-Collel, A., Whinston, M., Green, J.R. (1995). Microeconomic Theory. New York: Oxford University Press.

Ok, E. A. (2002). Utility representation of an incomplete preference relation. Journal of Economic Theory, 104:429-449. 
Rawls, J. (1971). A Theory of Justice. Cambridge: Bellknap Press of Harvard U. Press.

Redelmeier, D., Shafir, E. (1993). Medical Decision Making in Situations That Offer Multiple Alternatives. Journal of the American Medical Association, 273(4):302-305.

Rodriguez-Lara, I., Moreno-Garrido, L. (2011). Self-interest and fairness: self-serving choices of justice principles. Experimental Economics, 5:1-18.

Savage, L. (1954). The Foundations of Statistics. New York: Wiley.

Schlag, K., van der Weele, J. (2009). Efficient Interval Scoring Rules. Universitat Pompeu Fabra Working Paper 1176.

Schokkaert, E., Overlaet, B. (1989). Moral Intuitions and Economic Models of Distributive Justice. Social Choice and Welfare, 6(1):19-31.

Selten, R. and A. Ockenfels (1998). An Experimental Solidarity Game. Journal of Economic Behavior and Organization, 34:517-539.

Shafir, E. (1993). Choosing versus rejecting: why some options are both better and worse than others. Memory and Cognition, 21:546-556.

Shafir, E., Tversky, A. (1992). Thinking through uncertainty: nonconsequential reasoning and choice. Cognitive Psychology, 24:449-474.

Simonson, I. (1989). Choice Based on Reasons: The case of Attraction and Compromise Effects. Journal of Consumer Research, 16:158-174.

Sen, A. (1973). On Economic Inequality. Expanded Edition with a Substantial Annexe by James E. Foster and Amartya Sen. Oxford: Clarendon Press.

Sen. A. (1979). The Welfare Basis of Real Income Comparisons: A Survey. Journal of Economic Literature, 17(1):1-45.

Simonson, I. (1989). Choice Based on Reasons: The case of Attraction and Compromise Effects. Journal of Consumer Research, 16:158-174. 
Starmer, C., Sugden, R. (1991). Does the Random-Lottery Incentive System Elicit True Preferences? An Experimental Investigation. The American Economic Review, 81(4):971-978.

Thral, R., Rademacher, N. (2009). Bad luck vs. self-inflicted neediness. An experimental investigation of gift giving in a solidarity game. Journal of Economic Psychology, 30:517-526.

Tversky, A., Kahneman, D. (1979). Prospect Theory: An Analysis of Decision under Risk. Econometrica, 47(2):263-292.

Tversky, A., Kahneman, D. (1992). Advances in Prospect Theory: Cumulative Representation of Uncertainty. Journal of Risk and Uncertainty, 5:297323.

Tversky, A., Wakker, P. (1995). Risk Attitudes and Decision Weights. Econometrica, 63:1255-1280.

Wakker, P. (2010). Prospect Theory for Risk and Ambiguity. Cambridge: Cambridge University Press.

Wakker, P. (2008). Explaining the characteristics of the power (CRRA) utility family. Health Economics, 17:1329-1344.

Yaari, M. E., Bar-Hillel, M. (1984). On Dividing Justly. Social Choice and Welfare, 1(1):1-24. 


\section{Short Curriculum Vitae}

Elena Cettolin obtained a bachelor degree in Economics from the Free University of Trieste, Italy, in 2004. Thereafter she moved to Milan to further study economics at Bocconi University and completed a Master of Science in 2006. During the academic year 2006-2007 she worked as a teaching assistant at the department of Public Economics at Bocconi University and at the department of Microeconomics at the University of Bergamo. In September 2007 she moved to Maastricht to start her doctoral studies. In the fall semester of 2011 she has been visiting the CESS lab at New York University. Elena's research focuses on behavioral and experimental economics, decision theory and distributive justice. 
